\title{
Synthesis of Epoxyquinol A and Related Molecules: Probing Chemical Reactivity of Epoxyquinol Dimers and 2H-Pyran Precursors
}

\author{
Chaomin Li and John A. Porco, Jr.* \\ Department of Chemistry and Center for Chemical Methodology and \\ Library Development, \\ Boston University, Boston, Massachusetts 02215
}

\section{Supporting Information}

General Information: ${ }^{1} \mathrm{H}$ NMR spectra were recorded on a $400 \mathrm{MHz}$ spectrometer at ambient temperature with $\mathrm{CDCl}_{3}$ as the solvent unless otherwise stated. ${ }^{13} \mathrm{C}$ NMR spectra were recorded on a $75.0 \mathrm{MHz}$ spectrometer at ambient temperature. Chemical shifts are reported in parts per million relative to chloroform $\left({ }^{1} \mathrm{H}, \delta 7.24 ;{ }^{13} \mathrm{C}, \delta 77.00\right)$. Data for ${ }^{1} \mathrm{H}$ NMR are reported as follows: chemical shift, multiplicity $($ app $=$ apparent, $\mathrm{s}=$ singlet, $\mathrm{d}=$ doublet, $\mathrm{t}=$ triplet, $\mathrm{q}=$ quartet, $\mathrm{m}=$ multiplet, $\mathrm{br}=$ broad), integration and coupling constants. All ${ }^{13} \mathrm{C}$ NMR spectra were recorded with complete proton decoupling. Infrared spectra were recorded on a Nicolet Nexus 670 FT-IR spectrophotometer. Low and highresolution mass spectra were obtained in the Boston University Mass Spectrometry Laboratory using a Finnegan MAT-90 spectrometer. X-ray crystal structures for compounds 7, 8, 32, 55, 63 were obtained by Dr. Emil Lobkovsky (Department of Chemistry and Chemical Biology, Cornell University). Optical rotations were recorded on an AUTOPOL III digital polarimeter at $589 \mathrm{~nm}$, and are recorded as $[\alpha]_{\mathrm{D}}{ }^{22}$ (concentration in grams/100 mL solvent). Microwave reactions were performed using the Discover $^{\mathrm{TM}}$ Explorer System (CEM corp., matthews, NC). Analytical thin layer chromatography was performed using $0.25 \mathrm{~mm}$ silica gel plates. Flash chromatography was performed using 32-63 $\mu$ silica gel (Sorbent Tech. Inc.). Yields refer to chromatographically and spectroscopically pure materials, unless otherwise stated. Methylene chloride, tetrahydrofuran, toluene, methanol, benzene were purified by passing through two packed columns of neutral alumina (Glass Contour, Irvine, CA). Photochemistry experiments were performed using a Hanovia $450 \mathrm{~W}$ medium pressure mercury lamp housed in a watercooled quartz immersion well. All reactions were carried out in oven-dried glassware under an argon atmosphere unless otherwise noted.

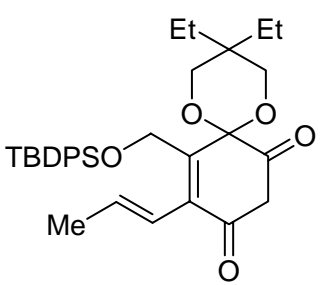

15

1,3-Diketone 15: $\mathrm{Pd}\left(\mathrm{PPh}_{3}\right)_{4}(15 \mathrm{mg}, 0.013 \mathrm{mmol})$ was placed in a 10 $\mathrm{mL}$ Schlenk flask. Next $50 \mathrm{mg}(0.085 \mathrm{mmol})$ of epoxide 12 dissolved in $1 \mathrm{~mL}$ of degassed toluene was added, followed by addition of $56 \mu \mathrm{L}(0.17 \mathrm{mmol})$ of E-tributyl-1-propenylstannane. The mixture was stirred at $100{ }^{\circ} \mathrm{C}$ for $1.5 \mathrm{~h}$, then cooled to $\mathrm{rt}$, concentrated, and directly subjected to silica gel chromatography (20 $\% \mathrm{Et}_{2} \mathrm{O}$ in hexane) to afford $30 \mathrm{mg}(0.055 \mathrm{mmol}, 65 \%)$ of diketone 15 as a pale yellow oil. ${ }^{1} \mathrm{H}$ NMR $\left(400 \mathrm{MHz} \mathrm{CDCl}_{3}\right) \delta 7.69(\mathrm{~m}$, 
4H), 7.44-7.36 (m, 6H), $6.27(\mathrm{dt}, 1 \mathrm{H}, J=6.4 \mathrm{~Hz}, 16 \mathrm{~Hz}), 6.12(\mathrm{~d}, 1 \mathrm{H}, J=16 \mathrm{~Hz}), 4.53(\mathrm{~s}$, 2H), $3.67(\mathrm{~m}, 4 \mathrm{H}), 3.55(\mathrm{~s}, 2 \mathrm{H}), 1.71(\mathrm{~d}, 3 \mathrm{H}, J=6.4 \mathrm{~Hz}), 1.53(\mathrm{q}, 2 \mathrm{H}, J=7.6 \mathrm{~Hz}), 1.04$ $(\mathrm{s}, 9 \mathrm{H}), 1.00(\mathrm{q}, 2 \mathrm{H}, J=0.76 \mathrm{~Hz}), 0.70(\mathrm{t}, 3 \mathrm{H}, J=7.6 \mathrm{~Hz}), 0.65(\mathrm{t}, 3 \mathrm{H}, J=7.6 \mathrm{~Hz}) ;{ }^{13} \mathrm{C}$ NMR $\left(75.0 \mathrm{MHz}, \mathrm{CDCl}_{3}\right) \delta 199.4,193.8,148.3,135.9,134.9,133.4,129.7,127.8,127.7$, $122.7,97.3,70.9,58.6,51.9,34.2,26.8,26.5,24.1,22.1,19.2,7.2,6.3$; IR (neat) vmax 2964, 2932, 2857, 1746, 1694, 1476, 1428, 1113, $1085 \mathrm{~cm}^{-1} ;$ CIHRMS [M+H] calculated for $\mathrm{C}_{33} \mathrm{H}_{43} \mathrm{O}_{5} \mathrm{Si}$ : 547.2882, found: 547.2885.

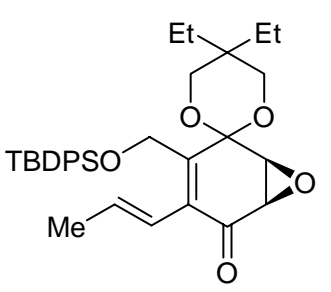

16

a-Propenyl enone 16: To a round-bottomed flask fitted with a water condenser was added chiral epoxide 12 (710 $\mathrm{mg}, 1.21 \mathrm{mmol})$ dissolved in $8 \mathrm{~mL}$ of degassed toluene. $520 \mathrm{mg}$ (1.576 mmol, 1.3 equiv) of $E$-tributyl-1-propenylstannane was added and the mixture was heated to $110^{\circ} \mathrm{C}$. In a separate flask, $55 \mathrm{mg}(0.06 \mathrm{mmol}, 0.05$ equiv) $\mathrm{Pd}_{2} \mathrm{dba}_{3}$ and $111 \mathrm{mg}\left(0.36 \mathrm{mmol}, 0.3\right.$ equiv) $\mathrm{AsPh}_{3}$ were stirred in $2 \mathrm{~mL}$ degassed toluene for $20 \mathrm{~min}$. The catalyst solution was then added to above reaction dropwise over $1 \mathrm{~h}$ and the reaction mixture was stirred for an additional $1 \mathrm{~h}$ at $110^{\circ} \mathrm{C}$. The solvent was removed by rotary evaporation and the residue directly subjected to silica gel chromatography (10\% EtOAc in hexane) to afford $650 \mathrm{mg}(1.19 \mathrm{mmol}, 98 \%)$ of 16 as a pale yellow oil. ${ }^{1} \mathrm{H} \mathrm{NMR}$ (400 $\left.\mathrm{MHz}, \mathrm{CDCl}_{3}\right) \delta 7.69(\mathrm{~m}, 4 \mathrm{H}), 6.30(\mathrm{~s}, 2 \mathrm{H}), 4.53(\mathrm{~d}, 1 \mathrm{H}, J=12 \mathrm{~Hz}), 4.47$ (d, 1H, J= 12 $\mathrm{Hz}), 4.33(\mathrm{~d}, 1 \mathrm{H}, J=4.8 \mathrm{~Hz}), 3.90(\mathrm{~d}, 1 \mathrm{H}, J=12 \mathrm{~Hz}), 3.80(\mathrm{~d}, 1 \mathrm{H}, J=12 \mathrm{~Hz}), 3.65$ (d, $1 \mathrm{H}, J=12 \mathrm{~Hz}), 3.55(\mathrm{~d}, 1 \mathrm{H}, J=4.8 \mathrm{~Hz}), 3.54(\mathrm{~d}, 1 \mathrm{H}, J=12 \mathrm{~Hz}), 1.75(\mathrm{~d}, 3 \mathrm{H}, J=4.8$ $\mathrm{Hz}), 1.35$ (q, 2H, $J=7.6 \mathrm{~Hz}), 1.10(\mathrm{q}, 2 \mathrm{H}, J=7.6 \mathrm{~Hz}), 1.04(\mathrm{~s}, 9 \mathrm{H}), 0.73(\mathrm{t}, 3 \mathrm{H}, J=7.6$ $\mathrm{Hz}), 0.58(\mathrm{t}, 3 \mathrm{H}, J=7.6 \mathrm{~Hz}) ;{ }^{13} \mathrm{C} \mathrm{NMR}\left(75.0 \mathrm{MHz}, \mathrm{CDCl}_{3}\right) \delta 195.1,144.6,136.0,134.2$, 133.6, 129.7, 127.7, 123.4, 94.8, 68.4, 59.1, 53.5, 50.0, 34.7, 28.4, 27.0, 25.2, 23.3, 19.6, 17.5, 13.7, 7.4, 6.7; IR (thin film) vmax 2964, 2932, 2859, 1692, 1468, 1112, $1090 \mathrm{~cm}^{-1}$; CIHRMS M ${ }^{+}$calculated for $\mathrm{C}_{33} \mathrm{H}_{42} \mathrm{O}_{5} \mathrm{Si}: 546.2802$, found: $546.2847 ;[\alpha]_{\mathrm{D}}^{22}=+70.4^{\mathrm{o}}(\mathrm{c}$ $\left.=1.0, \mathrm{CHCl}_{3}\right)$.

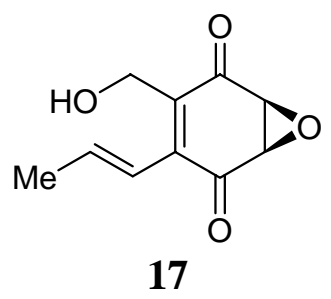

17

Quinone monoepoxide 17: Enone 16 (1.4 g, $2.56 \mathrm{mmol})$ was dissolved in $40 \mathrm{~mL} \mathrm{CH}_{3} \mathrm{CN}$ in a plastic bottle and $20 \mathrm{~mL} 48 \% \mathrm{HF}$ was added. The mixture was stirred at $\mathrm{rt}$ for $1 \mathrm{~h}$ before being quenched with sat. $\mathrm{NaHCO}_{3}$. The mixture was extracted with EtOAc, the organic extracts were combined, washed with $5 \%$ $\mathrm{NaHCO}_{3}$ and brine, dried over $\mathrm{MgSO}_{4}$, filtered, and concentrated in vacuo. Purification on silica gel (25\% EtOAc in hexane) provided $415 \mathrm{mg}(2.14 \mathrm{mmol}, 84 \%)$ of $\mathbf{1 7}$ as a golden yellow solid. M.p. 94-96 ${ }^{\circ} \mathrm{C} ;{ }^{1} \mathrm{H}$ NMR $\left(400 \mathrm{MHz}, \mathrm{CDCl}_{3}\right) \delta 6.53(\mathrm{dt}, 1 \mathrm{H}, J=6.4,16 \mathrm{~Hz}), 6.30(\mathrm{~d}, 1 \mathrm{H}$, $J=16 \mathrm{~Hz}), 4.48(\mathrm{dd}, 1 \mathrm{H}, J=7.2,12.8 \mathrm{~Hz}), 4.34(\mathrm{dd}, 1 \mathrm{H}, J=5.6,12.8 \mathrm{~Hz}), 3.84(\mathrm{~d}, 1 \mathrm{H}, J$ $=4.0 \mathrm{~Hz}), 3.80(\mathrm{~d}, 1 \mathrm{H}, J=4.0 \mathrm{~Hz}), 2.77(\mathrm{~s}, 1 \mathrm{H}), 1.89(\mathrm{~d}, 3 \mathrm{H}, J=6.4 \mathrm{~Hz}) ;{ }^{13} \mathrm{C} \mathrm{NMR}$ $\left(75.0 \mathrm{MHz}, \mathrm{CDCl}_{3}\right) \delta 193.8,193.3,142.4,141.5,137.1,122.0,57.0,54.1,53.8,20.2$; IR (thin film) vmax 3422, 2949, 1691, 1635, 1285, $1005 \mathrm{~cm}^{-1}$; CIHRMS calculated for $\mathrm{C}_{10} \mathrm{H}_{11} \mathrm{O}_{4}[\mathrm{M}+\mathrm{H}]^{+}: 195.0659$, found: $195.0668 ;[\alpha]_{\mathrm{D}}^{22}=-34.6^{\circ}\left(\mathrm{c}=1.0, \mathrm{CHCl}_{3}\right)$. 
<smiles>C/C=C/C1=C(CO)[C@@H](O)C2OC2C1=O</smiles><smiles>C/C=C/C1=C(CO)[C@@H](O)[C@H]2OC2C1=O</smiles>

Epoxyquinol A monomer 9 (RK302) and its epimer 18: To $75 \mathrm{mg}$ $(0.387 \mathrm{mmol})$ of $13 \mathrm{in} 5 \mathrm{~mL}$ THF was added $770 \mu \mathrm{L}(770 \mathrm{mmol}, 2.0$ equiv) of DIBAL-H (1.0 $\mathrm{M}$ in hexane) at $-78{ }^{\circ} \mathrm{C}$. The mixture was stirred for $10 \mathrm{~min}$ before being quenched with $5 \mathrm{~mL} 1 \mathrm{~N} \mathrm{HCl}$. The reaction mixture was extracted with $2: 1 \mathrm{CHCl}_{3} / \mathrm{PrOH}$. The combined organic layers were washed with brine, dried over $\mathrm{MgSO}_{4}$, filtered, and concentrated in vacuo. Purification on silica gel (50\% EtOAc in hexane) provided 54 $\mathrm{mg}(0.276 \mathrm{mmol}, 72 \%)$ of anti-epoxyquinol 9 and $3 \mathrm{mg}(0.015 \mathrm{mmol}, 4 \%)$ of a synepoxyquinol 18.

anti-Epoxyquinol 9: ${ }^{1} \mathrm{H} \mathrm{NMR}\left(400 \mathrm{MHz}, \mathrm{CDCl}_{3}\right) \delta 6.00(\mathrm{~d}, 1 \mathrm{H}, J=16 \mathrm{~Hz}), 5.94(\mathrm{dt}$, $1 \mathrm{H}, J=5.6,16 \mathrm{~Hz}), 4.95(\mathrm{~d}, 1 \mathrm{H}, J=4.8 \mathrm{~Hz}), 4.70$ (dd, 1H, $J=4.4,14 \mathrm{~Hz}), 4.43$ (dd, $1 \mathrm{H}$, $J=4.8,14 \mathrm{~Hz}), 3.80(\mathrm{~d}, 1 \mathrm{H}, J=4.0 \mathrm{~Hz}), 3.53(\mathrm{~d}, 1 \mathrm{H}, J=4.0 \mathrm{~Hz}), 3.49(\mathrm{~d}, 1 \mathrm{H}, J=4.8$ $\mathrm{Hz}), 2.51(\mathrm{t}, 1 \mathrm{H}, J=4.8 \mathrm{~Hz}), 1.81(\mathrm{~d}, 3 \mathrm{H}, J=5.6 \mathrm{~Hz}) ;{ }^{13} \mathrm{C} \mathrm{NMR}\left(75.0 \mathrm{MHz}, \mathrm{CDCl}_{3}\right) \delta$ 195.2, 147.1, 135.5, 131.1, 121.7, 65.2, 62.6, 55.9, 53.7, $19.3 \mathrm{~cm}^{-1}$; CIHRMS calculated for $\mathrm{C}_{10} \mathrm{H}_{13} \mathrm{O}_{4}[\mathrm{M}+\mathrm{H}]^{+}: 197.0816$, found: $197.0825 ; \quad[\alpha]_{\mathrm{D}}^{22}=+25.9^{\circ}\left(\mathrm{c}=1.0, \mathrm{CHCl}_{3}\right)$. Synthetic 9 was found to be identical with natural 9 (RK-302) by HPLC and TLC $\left(\mathrm{R}_{f}=\right.$ $\left.0.46, \mathrm{CHCl}_{3} / \mathrm{MeOH}=10 / 1\right)$.

syn-Epoxyquinol 18: ${ }^{1} \mathrm{H}$ NMR $\left(400 \mathrm{MHz}, \mathrm{CDCl}_{3}\right) \delta 6.04(\mathrm{~d}, 1 \mathrm{H}, J=15.6 \mathrm{~Hz}), 5.90(\mathrm{dt}$, $1 \mathrm{H}, J=5.6,15.6 \mathrm{~Hz}), 4.83(\mathrm{~s}, 1 \mathrm{H}), 4.52(\mathrm{~d}, 1 \mathrm{H}, J=14.4 \mathrm{~Hz}), 4.49$ (dd, $1 \mathrm{H}, J=14.4 \mathrm{~Hz}$ ), $3.86(\mathrm{~d}, 1 \mathrm{H}, J=4.0 \mathrm{~Hz}), 3.53(\mathrm{~d}, 1 \mathrm{H}, J=4.0 \mathrm{~Hz}), 3.25(\mathrm{~s}, 1 \mathrm{H}), 1.79(\mathrm{~d}, 3 \mathrm{H}, J=5.6 \mathrm{~Hz})$; ${ }^{13} \mathrm{C}$ NMR $\left(75.0 \mathrm{MHz}, \mathrm{CDCl}_{3}\right) \delta 194.2,147.8,135.1,130.3,121.9,66.8,61.4,54.8,54.2$, $19.3 \mathrm{~cm}^{-1}$; IR (thin film) vmax 3388, 3026, 2918, 2855, 1680,1446, 1359, $1046 \mathrm{~cm}^{-}$ ${ }^{1}$; CIHRMS calculated for $\mathrm{C}_{10} \mathrm{H}_{13} \mathrm{O}_{4}[\mathrm{M}+\mathrm{H}]^{+}: 197.0816$, found: $197.0828 ;[\alpha]_{\mathrm{D}}{ }^{22}=$ $+144.3^{\circ}\left(\mathrm{c}=1.0, \mathrm{CHCl}_{3}\right)$.

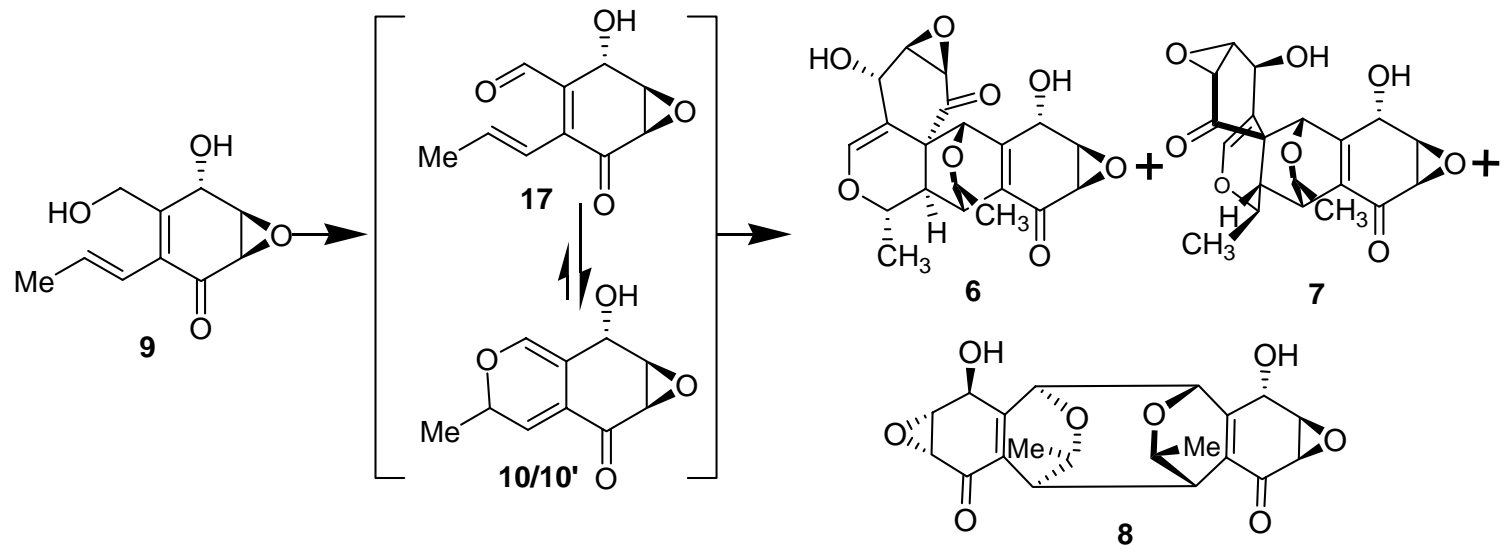

Epoxyquinol A (6), epoxyquinol B (7) and RKB-3564 D (8): $15 \mathrm{mg}$ (0.077 mmol) of 9, TEMPO (1.8 mg, $0.012 \mathrm{mmol}, 0.15$ equiv) and $\mathrm{CuCl}$ (1.1 $\mathrm{mg}, 0.011 \mathrm{mmol}, 0.14$ equiv) 
were dissolved in $1 \mathrm{~mL}$ of DMF and stirred at $\mathrm{rt}$ for $3 \mathrm{~h}$ under $\mathrm{O}_{2}(1 \mathrm{~atm})$. After addition of $2 \mathrm{~mL}$ of sat. $\mathrm{CuSO}_{4}$, the reaction mixture was extracted with EtOAc. The combined organic layers were washed with brine, dried over $\mathrm{MgSO}_{4}$, filtered, and concentrated in vacuo. The residue was dissolved in $5 \mathrm{~mL}$ of $40 \% \mathrm{MeOH}$ in $\mathrm{H}_{2} \mathrm{O}$ and stirred for $10 \mathrm{~h}$ at rt. The solution was then concentrated and subjected to silica gel chromatography (30:30:1 of $\mathrm{CH}_{2} \mathrm{Cl}_{2}: \mathrm{CHCl}_{3}: \mathrm{MeOH}$ ) to provide $8.2 \mathrm{mg}(0.021 \mathrm{mmol}, 55.2 \%$ ) of $\mathbf{6}, 2.0 \mathrm{mg}$ $(0.005 \mathrm{mmol}, 13.5 \%)$ of dimer 7 as a white solid and less than $0.5 \mathrm{mg}$ of $\mathbf{8}$.

Epoxyquinol A (6). ${ }^{1} \mathrm{H}$ NMR $\left(400 \mathrm{MHz}\right.$, acetone- $\left.\mathrm{d}_{6}\right) \delta 6.72(\mathrm{~d}, 1 \mathrm{H}, J=2.0 \mathrm{~Hz}), 5.22(\mathrm{~s}$, $1 \mathrm{H}), 4.94(\mathrm{~s}, 1 \mathrm{H}), 4.69(\mathrm{~s}, 1 \mathrm{H}), 4.43(\mathrm{dq}, 1 \mathrm{H}, J=1.2,6.4 \mathrm{~Hz}), 4.30(\mathrm{dq}, 1 \mathrm{H}, J=1.2,6.4$ $\mathrm{Hz}), 3.75(\mathrm{~d}, 1 \mathrm{H}, J=3.6 \mathrm{~Hz}), 3.74(\mathrm{~d}, 1 \mathrm{H}, J=3.6 \mathrm{~Hz}), 3.44(\mathrm{~d}, 1 \mathrm{H}, J=3.6 \mathrm{~Hz}), 3.39$ (d, $1 \mathrm{H}, J=3.6 \mathrm{~Hz}), 3.10(\mathrm{dd}, 1 \mathrm{H}, J=1.2,1.2 \mathrm{~Hz}), 2.44(\mathrm{~s}, 1 \mathrm{H}), 1.00(\mathrm{~d}, 3 \mathrm{H}, J=3.6 \mathrm{~Hz})$, $0.71(\mathrm{~d}, 3 \mathrm{H}, J=6.4 \mathrm{~Hz}) ;{ }^{13} \mathrm{C}$ NMR $(75.0 \mathrm{MHz}$, acetone-d6) $\delta 200.75,190.22,153.55$, $142.42,134.38,115.03,74.78,72.39,67.01,66.66,64.14,63.90,58.76,56.35,53.62$, 50.64, 39.28, 38.00, 20.96, 20.27; IR (thin film) vmax 3423, 2978, 1709, 1676, 1379, 1244, 1202, $1041 \mathrm{~cm}^{-1}$; CIHRMS $\mathrm{M}^{+}$calculated for $\mathrm{C}_{20} \mathrm{H}_{20} \mathrm{O}_{8}: 388.1158$, found: $388.1119 ;[\alpha]_{\mathrm{D}}^{22}=+66^{\circ}\left(\mathrm{c}=1.0, \mathrm{CHCl}_{3}\right)$.

Synthetic and natural epoxyquinol $\mathrm{A}$ gave the same $\mathrm{R}_{f}$ value in the following three solvent systems: $33 \% \mathrm{CH}_{2} \mathrm{Cl}_{2}-67 \%$ EtOAc $\left(\mathrm{R}_{f} 0.39\right), 20 \%$ hexane $-80 \% \mathrm{Et}_{2} \mathrm{O}\left(\mathrm{R}_{f}\right.$ $0.14), 33 \%$ hexane $-67 \%$ EtOAc $\left(\mathrm{R}_{f} 0.27\right)$.

Epoxyquinol B (7). M.p. $210-212^{\circ} \mathrm{C} ;{ }^{1} \mathrm{H}$ NMR $\left(400 \mathrm{MHz}, \mathrm{CDCl}_{3}\right) \delta 6.47(\mathrm{~s}, 1 \mathrm{H}), 5.04$ (s, $1 \mathrm{H}), 4.81(\mathrm{~s}, 1 \mathrm{H}), 4.62(\mathrm{~s}, 1 \mathrm{H}), 4.14(\mathrm{q}, 1 \mathrm{H}, J=6.4 \mathrm{~Hz}), 3.80(\mathrm{dd}, 1 \mathrm{H}, J=1.2,3.6 \mathrm{~Hz})$, $3.60(\mathrm{dd}, 1 \mathrm{H}, J=1.6,3.6 \mathrm{~Hz}), 3.53-3.46(\mathrm{~m}, 3 \mathrm{H}), 3.09(\mathrm{dd}, 1 \mathrm{H}, J=0.8,2.4 \mathrm{~Hz}), 2.76(\mathrm{dd}$, $1 \mathrm{H}, J=2.8,6.4 \mathrm{~Hz}), 1.25(\mathrm{~d}, 3 \mathrm{H}, J=6.4 \mathrm{~Hz}), 0.77(\mathrm{~d}, 3 \mathrm{H}, J=6.4 \mathrm{~Hz}) ;{ }^{13} \mathrm{C}$ NMR $(75.0$ $\left.\mathrm{MHz}, \mathrm{CDCl}_{3}\right) \delta 199.04,191.01,150.26,150.10,133.10,106.00,74.66,73.40,70.69$, $68.60,64.02,56.41,54.83,52.76,51.87,42.07,37.01,20.25,19.61,14.39$; IR (thin film) vmax 3359, 3018, 2926, 1682,1457, 1380,1259, $1022 \mathrm{~cm}^{-1}$; CIHRMS [M+H] calculated for $\mathrm{C}_{20} \mathrm{H}_{21} \mathrm{O}_{8}: 389.1238$, found: $389.1199 ;[\alpha]_{\mathrm{D}}{ }^{22}=+121.3^{\circ}\left(\mathrm{c}=1.0, \mathrm{CHCl}_{3}\right)$.

RKB-3564 D (8). ${ }^{1} \mathrm{H}$ NMR (400 MHz, acetone-d6) $\delta 4.80$ (s, 2H), 4.60 (d, $2 \mathrm{H}, J=8.0$ $\mathrm{Hz}), 4.38(\mathrm{~d}, 2 \mathrm{H}, J=8.0 \mathrm{~Hz}), 4.19(\mathrm{q}, 2 \mathrm{H}, J=6.4 \mathrm{~Hz}), 3.85(\mathrm{~d}, 2 \mathrm{H}, J=3.6 \mathrm{~Hz}), 3.53$ (d, $2 \mathrm{H}, J=3.6 \mathrm{~Hz}), 3.21(\mathrm{~s}, 2 \mathrm{H}), 0.76(\mathrm{~d}, 6 \mathrm{H}, J=6.4 \mathrm{~Hz}) ;{ }^{13} \mathrm{C}$ NMR $(75.0 \mathrm{MHz}$, acetone-d6) $\delta$ 193.0, 155.8, 132.8, 81.6, 72.9, 66.2, 58.5, 53.5, 39.9, 23.0; IR (thin film) vmax 3398, 2941, 2833, 1708, 1675, 1456, 1374, 1230, $1032 \mathrm{~cm}^{-1}$; CIHRMS $[\mathrm{M}+\mathrm{H}]^{+}$calculated for $\mathrm{C}_{26} \mathrm{H}_{31} \mathrm{O}_{4} \mathrm{Si}: 388.1158$, found: $388.1133 ;[\alpha]_{\mathrm{D}}^{22}=+293^{\circ}(\mathrm{c}=0.15$, acetone). Natural RKB-3564 D: $[\alpha]_{\mathrm{D}}^{21}=+303.3^{\circ}(\mathrm{c}=0.184$, acetone $)$

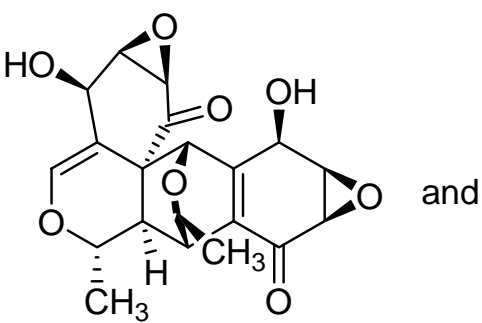

19

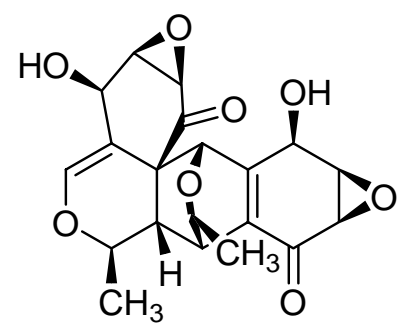

20
Dimers 19 and 20: $14 \mathrm{mg}$ (0.071 mmol) of 18, TEMPO ( $8 \mathrm{mg}, 0.08 \mathrm{mmol}, 1.1$ equiv) and $\mathrm{CuCl} \quad(12 \mathrm{mg}, \quad 0.077$ mmol, 1.1 equiv) were dissolved in $1 \mathrm{~mL}$ of DMF and stirred at $\mathrm{rt}$ for $1.5 \mathrm{~h}$ 
under $\mathrm{O}_{2}(1 \mathrm{~atm})$. After addition of $2 \mathrm{~mL}$ of water, the reaction mixture was extracted with EtOAc. The combined organic layers were washed with brine, dried over $\mathrm{MgSO}_{4}$, filtered, and concentrated in vacuo. The residue was subjected to silica gel chromatography $\left(20: 20: 1\right.$ of $\left.\mathrm{CH}_{2} \mathrm{Cl}_{2}: \mathrm{CHCl}_{3}: \mathrm{MeOH}\right)$ to provide $3.7 \mathrm{mg}(0.0095 \mathrm{mmol}, 26$ $\%)$ of dimer 19 as white solid and $2.6 \mathrm{mg}(0.0067 \mathrm{mmol}, 19 \%)$ of dimer 20 as white solid.

19: $\mathrm{mp} 120-122{ }^{\circ} \mathrm{C} .{ }^{1} \mathrm{H}$ NMR $\left(400 \mathrm{MHz}, \mathrm{CDCl}_{3}\right) \delta 6.69(\mathrm{~s}, 1 \mathrm{H}), 5.68(\mathrm{~s}, 1 \mathrm{H}), 4.67(\mathrm{~d}$, $1 \mathrm{H}, J=4.0 \mathrm{~Hz}), 4.30(\mathrm{~m}, 3 \mathrm{H}), 4.02(\mathrm{t}, 1 \mathrm{H}, J=3.6 \mathrm{~Hz}), 3.83(\mathrm{t}, 1 \mathrm{H}, J=3.2 \mathrm{~Hz}), 3.52(\mathrm{~d}$, $1 \mathrm{H}, J=4.0 \mathrm{~Hz}), 3.44(\mathrm{~d}, 1 \mathrm{H}, J=3.6 \mathrm{~Hz}), 3.08(\mathrm{~s}, 1 \mathrm{H}), 2.37(\mathrm{~s}, 1 \mathrm{H}), 1.05(\mathrm{~d}, 3 \mathrm{H}, J=6.4$ $\mathrm{Hz}), 0.84(\mathrm{~d}, 3 \mathrm{H}, J=6.0 \mathrm{~Hz}) ;{ }^{13} \mathrm{C}$ NMR $\left(75.0 \mathrm{MHz}, \mathrm{CDCl}_{3}\right) \delta 201.4,189.1,152.0$, $147.7,132.5,113.3,74.7,70.1,67.1,65.6,64.3,61.2,55.7,54.6,53.9,51.0,38.2,37.2$, 20.4, 19.8; IR (thin film) vmax 3406, 2926, 2854, 1709, 1685, 1457, 1408, 1379, 1242, 1206, $1084 \mathrm{~cm}^{-1}$; CIHRMS $[\mathrm{M}+\mathrm{H}]^{+}$calculated for $\mathrm{C}_{20} \mathrm{H}_{20} \mathrm{O}_{8}: 388.1158$, found: $388.1238 ;[\alpha]_{\mathrm{D}}^{22}=+79.4^{\circ}\left(\mathrm{c}=1.0, \mathrm{CHCl}_{3}\right)$.

20: $\mathrm{mp} 145-149{ }^{\circ} \mathrm{C} .{ }^{1} \mathrm{H}$ NMR $\left(400 \mathrm{MHz}, \mathrm{CDCl}_{3}\right) \delta 6.37$ (d, $\left.1 \mathrm{H}, J=2.4 \mathrm{~Hz}\right), 4.88$ (br s, $1 \mathrm{H}), 4.72(\mathrm{~s}, 1 \mathrm{H}), 4.75(\mathrm{br} \mathrm{s}, 1 \mathrm{H}), 4.14(\mathrm{q}, 1 \mathrm{H}, J=6.4 \mathrm{~Hz}), 3.88(\mathrm{~m}, 3 \mathrm{H}), 3.73(\mathrm{~d}, 1 \mathrm{H})$, $3.54(\mathrm{~d}, 2 \mathrm{H}, J=4.0 \mathrm{~Hz}), 3.06(\mathrm{~s}, 1 \mathrm{H}), 2.69(\mathrm{~s}, 1 \mathrm{H}), 1.11(\mathrm{~d}, 3 \mathrm{H}, J=6.4 \mathrm{~Hz}), 0.82(\mathrm{~d}, 3 \mathrm{H}$, $J=6.4 \mathrm{~Hz}) ;{ }^{13} \mathrm{C}$ NMR $\left(75.0 \mathrm{MHz}, \mathrm{CDCl}_{3}\right) \delta 198.2,189.4,151.0,141.6,129.9,106.7$, $72.9,72.5,68.6,66.4,66.3,56.6,54.4,54.3,52.4,42.5,38.0,19.9,19.4$; IR (thin film) vmax 3405, 2975, 2927, 1717, 1685, 1382, 1241, 1203, $1039 \mathrm{~cm}^{-1}$; CIHRMS M${ }^{+}$ calculated for $\mathrm{C}_{20} \mathrm{H}_{20} \mathrm{O}_{8}: 388.1158$, found: $388.1196 ;[\alpha]_{\mathrm{D}}^{22}=+218^{\circ}\left(\mathrm{c}=0.5, \mathrm{CHCl}_{3}\right)$.

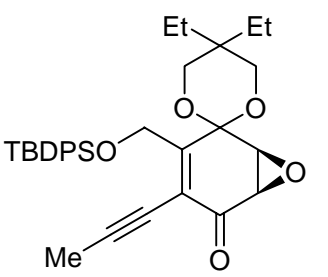

22

$\alpha$-Propynyl enone 22: To a round-bottomed flask fitted with a water condenser was added chiral epoxide $12(54 \mathrm{mg}, 0.092$ mmol) dissolved in $1 \mathrm{~mL}$ of degassed toluene. $70 \mathrm{mg}(0.21$ mmol, 2.5 equiv) of tributyl-1-propynylstannane was added and the mixture was heated to $90^{\circ} \mathrm{C}$. In a separate flask, $9 \mathrm{mg}(0.009$ mmol, 0.1 equiv) $\mathrm{Pd}_{2} \mathrm{dba}_{3} \mathrm{CHCl}_{3}$ and $16 \mathrm{mg}(0.052 \mathrm{mmol}, 0.6$ equiv) $\mathrm{AsPh}_{3}$ were stirred in $0.5 \mathrm{~mL}$ degassed toluene for 20 $\mathrm{min}$. The catalyst solution was then added to the above reaction dropwise and the reaction mixture was stirred for $2 \mathrm{~h}$ at $90^{\circ} \mathrm{C}$. The solvent was removed by rotary evaporation and the residue directly subjected to silica gel chromatography (10\% EtOAc in hexane) to afford $46 \mathrm{mg}(0.085 \mathrm{mmol}, 92 \%)$ of 22 as pale yellow oil. ${ }^{1} \mathrm{H}$ NMR (400 MHz, CDCl $) \delta 7.72(\mathrm{~m}, 4 \mathrm{H}), 7.38(\mathrm{~m}, 6 \mathrm{H}), 4.59(\mathrm{~s}, 2 \mathrm{H}), 4.35(\mathrm{~d}, 1 \mathrm{H}, J=4.0$ $\mathrm{Hz}), 3.92(\mathrm{~d}, 1 \mathrm{H}, J=12 \mathrm{~Hz}), 3.84(\mathrm{~d}, 1 \mathrm{H}, J=12 \mathrm{~Hz}), 3.73(\mathrm{~d}, 1 \mathrm{H}, J=12 \mathrm{~Hz}), 3.63(\mathrm{~d}$, $1 \mathrm{H}, J=12 \mathrm{~Hz}), 3.58(\mathrm{~d}, 1 \mathrm{H}, J=4.0 \mathrm{~Hz}), 1.88(\mathrm{~s}, 3 \mathrm{H}), 1.44(\mathrm{~m}, 2 \mathrm{H}), 1.14(\mathrm{q}, 2 \mathrm{H}, J=7.6$ $\mathrm{Hz}), 1.06(\mathrm{~s}, 9 \mathrm{H}), 0.75(\mathrm{t}, 3 \mathrm{H}, J=7.6 \mathrm{~Hz}), 0.61(\mathrm{t}, 3 \mathrm{H}, J=7.6 \mathrm{~Hz}) ;{ }^{13} \mathrm{C} \mathrm{NMR} \quad(75.0$ $\left.\mathrm{MHz}, \mathrm{CDCl}_{3}\right) \delta 191.3,154.0,135.8,133.7,133.6,129.5,127.5,122.9,96.9,94.1,72.6$, $68.3,68.2,60.6,52.6,50.1,34.5,26.6,24.8,22.9,19.4,7.1,6.4,4.7$; IR (thin film) vmax 2966, 2932, 2859, 1700,1428, 1217, $1112 \mathrm{~cm}^{-1}$; CIHRMS $[\mathrm{M}+\mathrm{H}]^{+}$calculated for $\mathrm{C}_{33} \mathrm{H}_{41} \mathrm{O}_{5} \mathrm{Si}: 545.2725$, found: $545.2647 ;[\alpha]_{\mathrm{D}}^{22}=+40^{\circ}\left(\mathrm{c}=1.0, \mathrm{CHCl}_{3}\right)$. 


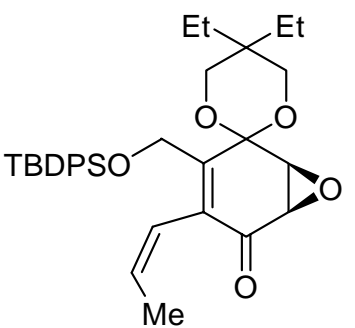

23

cis-Alkene 23: A suspension of $22(75 \mathrm{mg}, 0.138 \mathrm{mmol})$, quinoline (3 $\mu \mathrm{L}, 0.023 \mathrm{mmol})$, and commercially available Lindlar catalyst $(96 \mathrm{mg}, 5 \% \mathrm{w} / \mathrm{w} \mathrm{Pd}$ poisoned with $\mathrm{Pb}$ on $\mathrm{K}_{2} \mathrm{CO}_{3}$ ) in $10 \mathrm{~mL}$ of $\mathrm{CH}_{2} \mathrm{Cl}_{2}$ was stirred under an atmosphere of $\mathrm{H}_{2}$ for $5 \mathrm{~h}$, then another portion of Lindlar catalyst $(80 \mathrm{mg}$ ) was added and the reaction was stirred for another $2 \mathrm{~h}$. The reaction mixture was filtered though a funnel packed with silica gel and washed with EtOAc. The filtrate was concentrated and subjected to silica gel chromatography (25\% EtOAc in hexane) to afford $70 \mathrm{mg}(0.128 \mathrm{mmol}, 93 \%)$ of 23 as a pale yellow oil.

${ }^{1} \mathrm{H}$ NMR (400 MHz, CDCl 3 ) $\delta 7.67(\mathrm{~m}, 4 \mathrm{H}), 7.37(\mathrm{~m}, 6 \mathrm{H}), 5.91(\mathrm{~d}, 1 \mathrm{H}, J=11.2 \mathrm{~Hz}), 5.66$ $(\mathrm{dq}, 1 \mathrm{H}, J=11.2,6.8 \mathrm{~Hz}), 4.46(\mathrm{~d}, 1 \mathrm{H}, 12 \mathrm{~Hz}), 4.38(\mathrm{~m}, 2 \mathrm{H}), 3.95(\mathrm{~d}, 1 \mathrm{H}, J=12 \mathrm{~Hz})$, $3.90(\mathrm{~d}, 1 \mathrm{H}, J=12 \mathrm{~Hz}), 3.70(\mathrm{~d}, 1 \mathrm{H}, J=12 \mathrm{~Hz}), 3.62(\mathrm{~d}, 1 \mathrm{H}, J=12 \mathrm{~Hz}), 3.58(\mathrm{~d}, 1 \mathrm{H}, J=$ $4.8 \mathrm{~Hz}), 1.45$ (m, 1H), 1.39 (d, 3H, $J=6.8 \mathrm{~Hz}), 1.43$ (q, 2H, $J=7.6 \mathrm{~Hz}), 1.02(\mathrm{~s}, 9 \mathrm{H})$, $0.75(\mathrm{t}, 3 \mathrm{H}, J=7.6 \mathrm{~Hz}), 0.62(\mathrm{t}, 3 \mathrm{H}, J=7.6 \mathrm{~Hz}) ;{ }^{13} \mathrm{C} \mathrm{NMR}\left(75.0 \mathrm{MHz}, \mathrm{CDCl}_{3}\right) \delta 193.3$, $148.1,135.8,135.7,134.0,133.4,133.3,129.9,129.5,127.4,122.6,94.1,68.2,59.7$, $52.9,50.0,34.5,26.8,25.0,23.1,19.4,15.1,7.3,6.5$; IR (thin film) vmax 2965, 2932, $2858,1690,1463,1428,1132,1112,1011 \mathrm{~cm}^{-1}$; CIHRMS $[\mathrm{M}+\mathrm{H}]^{+}$calculated for $\mathrm{C}_{33} \mathrm{H}_{43} \mathrm{O}_{5} \mathrm{Si}: 547.2882$, found: 547.2886; $[\alpha]_{\mathrm{D}}{ }^{22}=+9.2^{\circ}\left(\mathrm{c}=1.0, \mathrm{CHCl}_{3}\right)$.

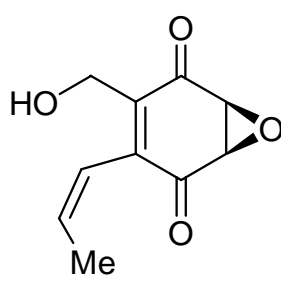

66

Quinone monoepoxide 66: 23 (70 $\mathrm{mg}, 0.128 \mathrm{mmol}$ ) was dissolved in $2 \mathrm{~mL}$ of $\mathrm{CH}_{3} \mathrm{CN}$ in a plastic bottle and $1 \mathrm{~mL} \mathrm{48 \%} \mathrm{HF} \mathrm{was} \mathrm{added.}$ The mixture was stirred at $\mathrm{rt}$ for $8 \mathrm{~h}$ before addition of 5 water. The reaction mixture was extracted with EtOAc, the organic extracts were combined, washed with water and brine, dried over $\mathrm{MgSO}_{4}$, filtered, and concentrated in vacuo. Purification on silica gel $(33 \%$ EtOAc in hexane) provided $19 \mathrm{mg}(0.098 \mathrm{mmol}, 76 \%)$ of $\mathbf{6 6}$ as a yellow oil. ${ }^{1} \mathrm{H}$ NMR (400 MHz, $\left.\mathrm{CDCl}_{3}\right) \delta 6.09(\mathrm{~m}, 2 \mathrm{H}), 4.37(\mathrm{~m}$, $2 \mathrm{H}), 3.88(\mathrm{~m}, 2 \mathrm{H}), 1.56(\mathrm{~d}, 3 \mathrm{H}, J=5.2 \mathrm{~Hz}) ;{ }^{13} \mathrm{C} \mathrm{NMR}\left(75.0 \mathrm{MHz}, \mathrm{CDCl}_{3}\right) \delta 194.0$, 192.0, 140.3, 135.4, 121.8, 120.4, 58.7, 54.0, 53.5, 15.7; IR (thin film) vmax 3384, 3031, 2965, 1698, 1347, 1293, $1024 \mathrm{~cm}^{-1}$; CIHRMS M${ }^{+}$calculated for $\mathrm{C}_{10} \mathrm{H}_{10} \mathrm{O}_{4}:$ 194.0579, found: 194.0542; $[\alpha]_{\mathrm{D}}^{22}=-48^{\circ}\left(\mathrm{c}=1.0, \mathrm{CHCl}_{3}\right)$.

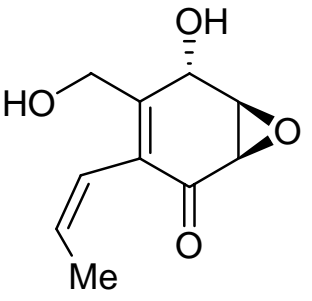

21

Epoxyquinol 21: To $19 \mathrm{mg}(0.098 \mathrm{mmol})$ of $\mathbf{6 6}$ in $2 \mathrm{~mL}$ of THF was added $196 \mu \mathrm{L}$ (196 mmol, 2.0 equiv) of DIBAL-H (1.0 M in hexane) at $-78{ }^{\circ} \mathrm{C}$. The mixture was stirred for $15 \mathrm{~min}$ before being quenched with $2 \mathrm{~mL} 1 \mathrm{~N} \mathrm{HCl}$. The reaction mixture was extracted with 2:1 $\mathrm{CHCl}_{3} / \mathrm{PrOH}$. The combined organic layers were washed with brine, dried over $\mathrm{MgSO}_{4}$, filtered, and concentrated in vacuo. Purification on silica gel (50\% EtOAc in hexane) provided $12 \mathrm{mg}(0.276 \mathrm{mmol}, 72 \%)$ of anti-epoxyquinol 21. ${ }^{1} \mathrm{H}$ NMR $\left(400 \mathrm{MHz}, \mathrm{CDCl}_{3}\right) \delta 5.88(\mathrm{~m}, 2 \mathrm{H}), 4.98(\mathrm{~s}, 1 \mathrm{H})$, $4.47(\mathrm{~d}, 1 \mathrm{H}, J=6.8 \mathrm{~Hz}), 4.32(\mathrm{~d}, 1 \mathrm{H}, J=6.8 \mathrm{~Hz}), 3.82(\mathrm{dd}, 1 \mathrm{H}, J=1.6,4.0 \mathrm{~Hz}), 3.54$ $(\mathrm{dd}, 1 \mathrm{H}, J=1.2,4.0 \mathrm{~Hz}), 1.44(\mathrm{~d}, 3 \mathrm{H}, J=5.2 \mathrm{~Hz}) ;{ }^{13} \mathrm{C} \mathrm{NMR}\left(75.0 \mathrm{MHz}, \mathrm{CDCl}_{3}\right) \delta$ 
193.9, 149.3, 132.2, 130.1, 121.2, 64.6, 63.2, 55.8, 53.0, 15.0; IR (thin film) vmax 3396, $3023,2918,1673,1405,1232,1041 \mathrm{~cm}^{-1}$; CIHRMS $[\mathrm{M}+\mathrm{H}]^{+}$calculated for $\mathrm{C}_{10} \mathrm{H}_{13} \mathrm{O}_{4}$ : 197.0816, found: $197.0801 ;[\alpha]_{\mathrm{D}}^{22}=+132^{\circ}\left(\mathrm{c}=1.0, \mathrm{CHCl}_{3}\right)$.

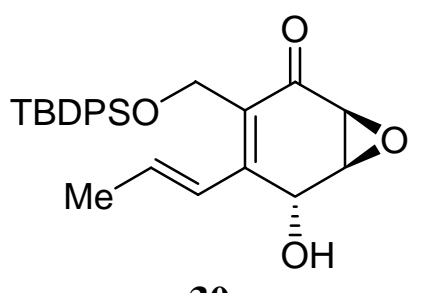

30

anti epoxy alcohol 34: To $700 \mathrm{mg}(1.28 \mathrm{mmol})$ of $\mathbf{1 6}$ in 10 $\mathrm{mL}$ THF was added $3.2 \mathrm{~mL}$ ( $3.2 \mathrm{mmol}, 2.5$ equiv) of DIBAL-H (1.0 $\mathrm{M}$ in hexane) at $-78{ }^{\circ} \mathrm{C}$. The mixture was stirred for $15 \mathrm{~min}$ before being quenched with $5 \mathrm{~mL} 1 \mathrm{~N}$ $\mathrm{HCl}$. The reaction mixture was extracted with 2:1 $\mathrm{CHCl}_{3} / \mathrm{iPrOH}$. The combined organic layers were washed with brine, dried over $\mathrm{MgSO}_{4}$, filtered, and concentrated in vacuo. The residue was dissolved in $10 \mathrm{~mL} \mathrm{CH}_{2} \mathrm{Cl}_{2}$ and 300 mg montmorillonite $\mathrm{K}-10$ clay was added. The mixture was stirred at $\mathrm{rt}$ for $5 \mathrm{~min}$ before filtrated and washed with EtOAc. The elute was concentrated and the residue was purified on silica gel $(20 \%$ EtOAc in hexane) to provide $500 \mathrm{mg}(1.15 \mathrm{mmol}, 90 \%)$ of anti-epoxyquinol $30 .{ }^{1} \mathrm{H}$ NMR (400 MHz, $\left.\mathrm{CDCl}_{3}\right) \delta 7.65(\mathrm{~m}, 4 \mathrm{H}), 7.39(\mathrm{~m}, 6 \mathrm{H}), 6.53(\mathrm{~d}, 1 \mathrm{H}, J=16 \mathrm{~Hz}), 6.43(\mathrm{dq}$, $1 \mathrm{H}, J=16,6.4 \mathrm{~Hz}), 4.98(\mathrm{~d}, 1 \mathrm{H}, J=8.4 \mathrm{~Hz}), 4.45(\mathrm{~d}, 1 \mathrm{H}, J=12 \mathrm{~Hz}), 4.40(\mathrm{~d}, 1 \mathrm{H}, J=12$ $\mathrm{Hz}), 3.76(\mathrm{dd}, 1 \mathrm{H}, J=1.6,3.6 \mathrm{~Hz}), 3.41(\mathrm{~d}, 1 \mathrm{H}, J=3.6 \mathrm{~Hz}), 2.50(\mathrm{~d}, 1 \mathrm{H}, J=8.4 \mathrm{~Hz})$, $1.85(\mathrm{~d}, 3 \mathrm{H}, J=6.4 \mathrm{~Hz}), 1.00(\mathrm{~s}, 9 \mathrm{H}) ;{ }^{13} \mathrm{C} \mathrm{NMR}\left(75.0 \mathrm{MHz}, \mathrm{CDCl}_{3}\right) \delta 194.1,148.3$, $136.45,135.66,133.16,129.9,129.7,127.7,127.6,63.13,56.1,55.2,52.3,26.7,19.5$, 19.2; IR (thin film) vmax 3418, 3049, 2932, 2857, 1666, 1631, 1428, 1111, $1046 \mathrm{~cm}^{-1}$; CIHRMS $[\mathrm{M}+\mathrm{H}]^{+}$calculated for $\mathrm{C}_{26} \mathrm{H}_{31} \mathrm{O}_{4} \mathrm{Si}: 435.1993$, found: $435.2008 ;[\alpha]_{\mathrm{D}}^{22}=-110^{\circ}$ $\left(\mathrm{c}=1.0, \mathrm{CHCl}_{3}\right)$.

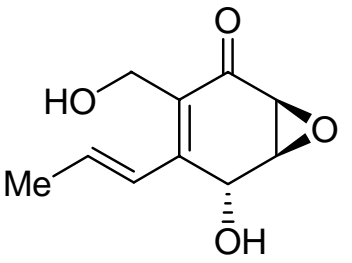

29

anti epoxy alcohol 29: 30 (500 $\mathrm{mg}, 1.15 \mathrm{mmol})$ was dissolved in $20 \mathrm{~mL} \mathrm{CH}_{3} \mathrm{CN}$ in a plastic bottle and $10 \mathrm{~mL} 48 \% \mathrm{HF}$ was added. The mixture was stirred at $\mathrm{rt}$ for $1 \mathrm{~h}$ before addition of $40 \mathrm{~mL}$ of water. The mixture was extracted with EtOAc, the organic extracts were combined, washed with water and brine, dried over $\mathrm{MgSO}_{4}$, filtered, and concentrated in vacuo. Purification on silica gel (25\% EtOAc in hexane) provided 174 $\mathrm{mg}(0.89 \mathrm{mmol}, \mathbf{7 7 \%})$ of $\mathbf{2 9}$ as a yellow oil. ${ }^{1} \mathrm{H}$ NMR (400 $\left.\mathrm{MHz}, \mathrm{CDCl}_{3}\right) \delta 6.47(\mathrm{~m}, 2 \mathrm{H}), 4.90(\mathrm{~d}, 1 \mathrm{H}, J=8.8 \mathrm{~Hz}), 4.48(\mathrm{~d}$, $1 \mathrm{H}, J=8.8 \mathrm{~Hz}), 4.22(\mathrm{~s}, 2 \mathrm{H}), 3.75(\mathrm{~d}, 1 \mathrm{H}, J=4.0 \mathrm{~Hz}), 3.38(\mathrm{~d}$, $1 \mathrm{H}, J=4.0 \mathrm{~Hz}), 1.87(\mathrm{~d}, 3 \mathrm{H}, J=5.6 \mathrm{~Hz}) ;{ }^{13} \mathrm{C} \mathrm{NMR}\left(75.0 \mathrm{MHz}, \mathrm{CDCl}_{3}\right) \delta 195.9,149.2$, 138.1, 129.0, 126.5, 62.6, 55.4, 55.0, 52.2, 19.5; IR (thin film) vmax 3379, 2910, 1664, 1630, 1441, 1385, $1042 \mathrm{~cm}^{-1}$; CIHRMS $[\mathrm{M}+\mathrm{H}]^{+}$calculated for $\mathrm{C}_{10} \mathrm{H}_{13} \mathrm{O}_{4}$ : 197.0816, found: $197.0829 ;[\alpha]_{\mathrm{D}}^{22}=-244^{\circ}\left(\mathrm{c}=1.0, \mathrm{CHCl}_{3}\right)$. 


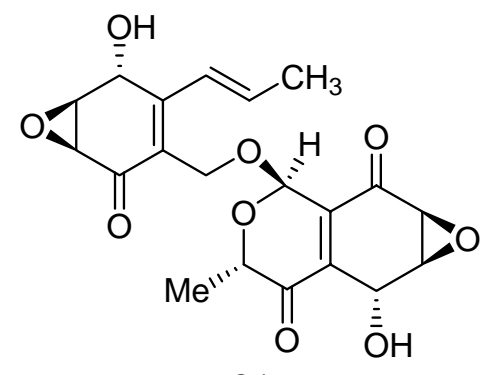

31

Pseudo dimer 31: $40 \mathrm{mg}(0.20 \mathrm{mmol})$ of 29, TEMPO (12.8 $\mathrm{mg}, 0.08)$ and $\mathrm{CuCl}(8 \mathrm{mg}, 0.08 \mathrm{mmol})$ were dissolved in $2 \mathrm{~mL}$ DMF and stirred at $\mathrm{rt}$ for $10 \mathrm{~h}$ under $\mathrm{O}_{2}$ (1 atm). After addition of $2 \mathrm{~mL}$ sat. $\mathrm{CuSO}_{4}$, the reaction mixture was extracted with EtOAc. The combined organic layers were washed with brine, dried over $\mathrm{MgSO}_{4}$, filtered, and concentrated in vacuo. The residue was subjected to silica gel chromatography (25\% EtOAc in hexane) to provide $20 \mathrm{mg}(0.05 \mathrm{mmol}$, $50 \%)$ of 31 as a pale yellow oil. ${ }^{1} \mathrm{H}$ NMR $(400 \mathrm{MHz}$, $\left.\mathrm{CDCl}_{3}\right) \delta 6.56(\mathrm{dq}, 1 \mathrm{H}, J=16,6.4 \mathrm{~Hz}), 6.45(\mathrm{~d}, 1 \mathrm{H}, J=16 \mathrm{~Hz}), 5.52(\mathrm{~s}, 1 \mathrm{H}), 5.11(\mathrm{~s}$, $1 \mathrm{H}), 5.02(\mathrm{~s}, 1 \mathrm{H}), 4.58(\mathrm{~d}, 1 \mathrm{H}, J=12 \mathrm{~Hz}), 4.54(\mathrm{q}, 1 \mathrm{H}, J=6.4 \mathrm{~Hz}), 4.49(\mathrm{~d}, 1 \mathrm{H}, J=12$ $\mathrm{Hz}), 3.86(\mathrm{~d}, 1 \mathrm{H}, J=2.0 \mathrm{~Hz}), 3.80(\mathrm{~d}, 1 \mathrm{H}, J=3.2 \mathrm{~Hz}), 3.56(\mathrm{~d}, 1 \mathrm{H}, J=3.6 \mathrm{~Hz}), 3.48(\mathrm{~d}$, $1 \mathrm{H}, J=3.6 \mathrm{~Hz}), 1.92(\mathrm{~d}, 3 \mathrm{H}, J=6.4 \mathrm{~Hz}), 1.40(\mathrm{~d}, 3 \mathrm{H}, J=7.2 \mathrm{~Hz}) ;{ }^{13} \mathrm{C}$ NMR $(75.0$ $\left.\mathrm{MHz}, \mathrm{CDCl}_{3}\right) \delta 199.1,194.3,150.5,138.5,137.6,137.2,127.2,127.0,89.7,69.9,63.2$, 60.2, 59.2, 55.9, 55.3, 52.4, 52.3, 19.6, 15.3; IR (thin film) vmax 3447, 3020, 2938, 1693, $1631,1377,1235,1041 \mathrm{~cm}^{-1}$; CILRMS $[\mathrm{M}+\mathrm{H}]^{+}$calculated for $\mathrm{C}_{20} \mathrm{H}_{21} \mathrm{O}_{9}: 405.4$, found: $405.3 ;[\alpha]_{\mathrm{D}}^{22}=-230^{\circ}\left(\mathrm{c}=1.0, \mathrm{CHCl}_{3}\right)$.

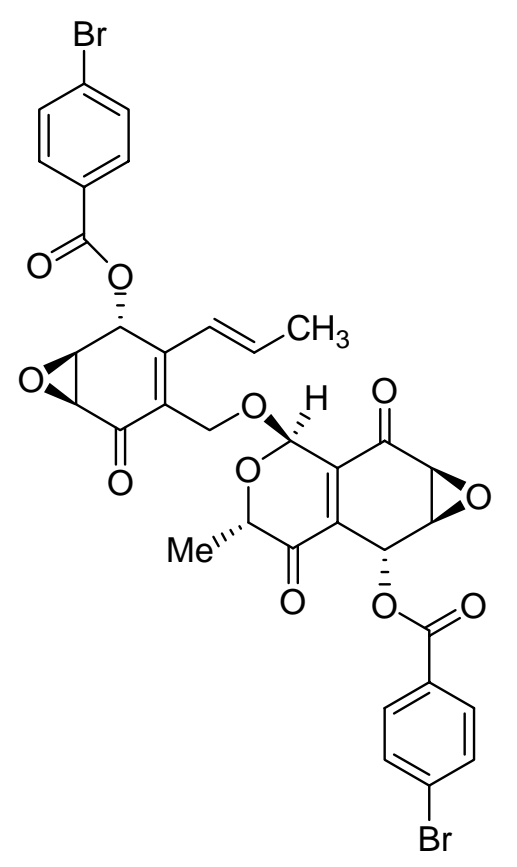

32
Bis-bromobenzoates 32: $11 \mathrm{mg}(0.028 \mathrm{mmol})$ of 31 and $12.4 \mathrm{mg} \quad(0.057 \mathrm{mmol}, 2$ equiv. $)$ of $4-$ bromobenzoyl chloride were dissolved in $1 \mathrm{~mL}$ of $\mathrm{CH}_{2} \mathrm{Cl}_{2}$, and $0.4 \mathrm{mg}$ of DMAP ( $0.003 \mathrm{mmol}, 0.1$ equiv) and $7.9 \mu \mathrm{L}$ of triethylamine $(0.057 \mathrm{mmol}, 2$ equiv. $)$ were added at $0^{\circ} \mathrm{C}$. After stirring at $0^{\circ} \mathrm{C}$ for $35 \mathrm{~min}, 5$ $\mathrm{mL}$ of water and $5 \mathrm{~mL}$ of $\mathrm{CH}_{2} \mathrm{Cl}_{2}$ were added. The water layer was washed with $3 \times 2 \mathrm{~mL}$ of $\mathrm{CH}_{2} \mathrm{Cl}_{2}$ and the combined organic layers were washed with brine, dried over $\mathrm{MgSO}_{4}$, filtered, and concentrated in vacuo. The residue was subjected to silica gel chromatography (1:4 hexane: $\mathrm{CH}_{2} \mathrm{Cl}_{2}$ then pure $\mathrm{CH}_{2} \mathrm{Cl}_{2}$ ) to afford 11.6 $\mathrm{mg}(0.015 \mathrm{mmol}, 54 \%)$ of bis-p-bromobenzoate 32 as white solid. mp 136-138 ${ }^{\circ} \mathrm{C} .{ }^{1} \mathrm{H} \quad \mathrm{NMR}(400 \mathrm{MHz}$, $\left.\mathrm{CDCl}_{3}\right) \delta 7.90(\mathrm{~d}, 2 \mathrm{H}, J=8.8 \mathrm{~Hz}), 7.81(\mathrm{~d}, 2 \mathrm{H}, J=8.8$ $\mathrm{Hz}), 7.57(\mathrm{~m}, 4 \mathrm{H}), 6.50(\mathrm{~m}, 2 \mathrm{H}), 5.77(\mathrm{~s}, 1 \mathrm{H}), 4.83(\mathrm{~d}$, $1 \mathrm{H}, J=11.2 \mathrm{~Hz}), 4.51(\mathrm{~m}, 2 \mathrm{H}), 3.93(\mathrm{~m}, 1 \mathrm{H}), 3.86(\mathrm{~m}$, $1 \mathrm{H}), 3.61(\mathrm{~d}, 1 \mathrm{H}, J=3.6 \mathrm{~Hz}), 3.58(\mathrm{~d}, 1 \mathrm{H}, J=3.6 \mathrm{~Hz})$, $1.87(\mathrm{~d}, 3 \mathrm{H}, J=6.8 \mathrm{~Hz}), 1.40(\mathrm{~d}, 3 \mathrm{H}, J=6.8 \mathrm{~Hz}) ;{ }^{13} \mathrm{C}$ NMR $\left(75.0 \mathrm{MHz}, \mathrm{CDCl}_{3}\right) \delta 196.5,193.5,192.6$, 164.9, 164.3, 145.6, 140.4, 137.7, 133.5, 129.2, 129.1, 127.5, 127.4, 126.6, 113.7, 90.8, $70.4,64.2,61.2,60.8,53.7,52.9,52.4,52.0,19.8,15.5$; IR (thin film) vmax 2828, 2856, $1729,1696,1590,1258,1096,1012 \mathrm{~cm}^{-1}$; CIHRMS $[\mathrm{M}+\mathrm{H}]^{+}$calculated for $\mathrm{C}_{34} \mathrm{H}_{26} \mathrm{Br}_{2} \mathrm{O}_{11}: 767.9842$, found: $767.9748 ;[\alpha]_{\mathrm{D}}^{22}=152^{\circ}\left(\mathrm{c}=0.5, \mathrm{CHCl}_{3}\right)$. 
<smiles>CC1C=C2C(=CO1)C(=O)C1OC1[C@@H]2O</smiles>

$34 / 34$ '

34/34': $20 \mathrm{mg}$ of Dess-Martin periodinane was added to $10 \mathrm{mg}$ ( $0.05 \mathrm{mmol}$ ) of 29 in $1 \mathrm{~mL}$ of $\mathrm{CH}_{2} \mathrm{Cl}_{2}$. The mixture was stirred at $\mathrm{rt}$ for $1 \mathrm{~h}$. The solvent volume was reduced by evaporation with an Argon flow. The residue was subjected to silica gel chromatography (30\% EtOAc in hexane) to provide $9 \mathrm{mg}$ (0.046 mmol, $90 \%$ of 34/34' as yellow oil. ${ }^{1} \mathrm{H}$ NMR analysis of the crude product showed as an 4:1 mixture of two diastereomers, which changed to $1: 1$ upon storage. ${ }^{1} \mathrm{H}$ NMR $\left(400 \mathrm{MHz}, \mathrm{CDCl}_{3}\right) \delta 7.54(\mathrm{~s}, 1 \mathrm{H}), 7.46(\mathrm{~s}, 1 \mathrm{H}), 5.38(\mathrm{~d}, 1 \mathrm{H}, J=4.0 \mathrm{~Hz}), 5.25(\mathrm{~s}, 1 \mathrm{H})$, $5.04(\mathrm{~m}, 2 \mathrm{H}), 4.69(\mathrm{~s}, 2 \mathrm{H}), 3.70(\mathrm{~m}, 2 \mathrm{H}), 3.37(\mathrm{~m}, 2 \mathrm{H}), 1.46(\mathrm{~d}, 3 \mathrm{H}, J=6.4 \mathrm{~Hz}), 1.37(\mathrm{~d}$, $3 \mathrm{H}, J=6.4 \mathrm{~Hz}) ;{ }^{13} \mathrm{C} \mathrm{NMR}\left(75.0 \mathrm{MHz}, \mathrm{CDCl}_{3}\right) \delta 190.3,190.2,156.8,155.3,131.2$, $129.4,119.2$, 119.0, 109.8, 108.7, 74.6, 74.5, 66.1, 66.0, 57.2, 57.1, 53.8, 53.7, 21.0, 20.8; IR (thin film) vmax 3420, 2978, 2926, 2855, 1683, 1646, 1568, 1206, $1029 \mathrm{~cm}^{-1}$; CIHRMS M ${ }^{+}$calculated for $\mathrm{C}_{10} \mathrm{H}_{10} \mathrm{O}_{4}: 194.0579$, found: $194.0587 ;[\alpha]_{\mathrm{D}}^{22}=-81^{\circ}(\mathrm{c}=$ $\left.1.0, \mathrm{CHCl}_{3}\right)$.
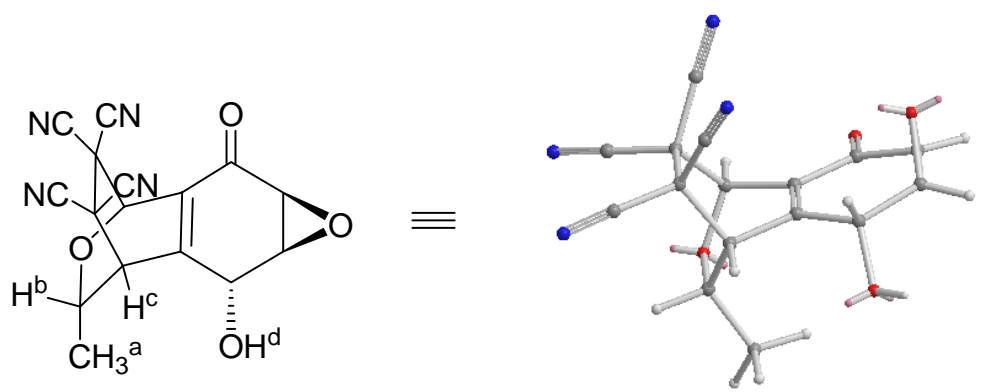

36

Cycloadduct 36: $10 \mathrm{mg}(0.05 \mathrm{mmol})$ of 34/34' and $20 \mathrm{mg}(0.156 \mathrm{mmol})$ of tetracyanoethylene were dissolved in $1 \mathrm{~mL}$ of $1: 1$ mixture of $\mathrm{PhCH}_{3} / \mathrm{THF}$ and the mixture was heated at $65{ }^{\circ} \mathrm{C}$ for $2 \mathrm{~h}$ and concentrated in vacuo. The residue was subjected to silica gel chromatography (40\% EtOAc in hexane) to provide $12 \mathrm{mg}(0.037 \mathrm{mmol}, 72 \%)$ of 36. ${ }^{1} \mathrm{H}$ NMR (400 MHz, acetone- $\left.\mathrm{d}_{6}\right) \delta 5.92(\mathrm{~s}, 1 \mathrm{H}), 5.71(\mathrm{~d}, 1 \mathrm{H}, J=8.4 \mathrm{~Hz}), 4.94(\mathrm{~d}, 1 \mathrm{H}$, $J=8.4 \mathrm{~Hz}), 4.66(\mathrm{dq}, 1 \mathrm{H}, J=1.6,6.4 \mathrm{~Hz}), 4.46(\mathrm{~d}, 1 \mathrm{H}, J=1.2 \mathrm{~Hz}), 4.01(\mathrm{~m}, 1 \mathrm{H}), 3.67$ $(\mathrm{dd}, 1 \mathrm{H}, J=1.2,4.0 \mathrm{~Hz}), 1.20(\mathrm{~d}, 3 \mathrm{H}, J=6.4 \mathrm{~Hz}) ; \quad$ NOED (400 MHz, acetone-d6) Irradiation at $\delta 1.20(\mathrm{H}-\mathrm{a}): 4.8 \%$ enhancement at $\mathrm{H}-\mathrm{b}, 2.2 \%$ enhancement at $\mathrm{H}-\mathrm{c}, 1.8 \%$ enhancement at $\mathrm{H}-\mathrm{d} ; \quad{ }^{13} \mathrm{C} \mathrm{NMR}\left(75.0 \mathrm{MHz}, \mathrm{CDCl}_{3}\right) \delta 188.2,152.6,133.5,112.3$, $112.1,112.0,111.1,69.4,67.6,65.3,59.0,53.8,46.9,41.8,20.6$; IR (thin film) vmax 3456, 2989, 2935, 1694, 1408, 1384, 1259, $1059 \mathrm{~cm}^{-1}$; CILRMS M${ }^{+}$calculated for $\mathrm{C}_{16} \mathrm{H}_{9} \mathrm{~N}_{4} \mathrm{O}_{4}$ : 321.1, found: $321.1 ;[\alpha]_{\mathrm{D}}^{22}=-110^{\circ}(\mathrm{c}=1.0$, acetone).

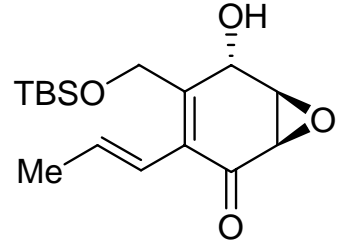

37
Silyl ether 37: To monomer 9 (45 mg, $0.23 \mathrm{mmol})$ in $2 \mathrm{~mL}$ of DMF was added $50 \mathrm{mg}(0.33 \mathrm{mmol})$ of TBSCl and $24 \mathrm{mg}(0.35$ mmol) of imidazole. The mixture was stirred at $\mathrm{rt}$ for $1 \mathrm{~h}, 5 \mathrm{~mL}$ of water was added, and the mixture was extracted with EtOAc. The organic extracts were combined, washed with brine, dried over $\mathrm{MgSO}_{4}$, filtered, and concentrated in vacuo. Purification on 
silica gel (15\% EtOAc in hexane) provided $69 \mathrm{mg}(0.22 \mathrm{mmol}, 97 \%)$ of compound 37 as a colorless oil; ${ }^{1} \mathrm{H}$ NMR $\left(400 \mathrm{MHz}, \mathrm{CDCl}_{3}\right) \delta 6.01(\mathrm{~d}, 1 \mathrm{H}, J=16 \mathrm{~Hz}), 5.85(\mathrm{dq}, 1 \mathrm{H}, J=$ 6.4, $16 \mathrm{~Hz}), 4.90(\mathrm{~s}, 1 \mathrm{H}), 4.70(\mathrm{~d}, 1 \mathrm{H}, J=14.4 \mathrm{~Hz}), 4.40(\mathrm{~d}, 1 \mathrm{H}, J=14.4 \mathrm{~Hz}), 3.78$ (dd, $1 \mathrm{H}, J=1.2,4.0 \mathrm{~Hz}), 3.53(\mathrm{~d}, 1 \mathrm{H}, J=4.0 \mathrm{~Hz}), 1.81(\mathrm{~d}, 3 \mathrm{H}, J=6.4 \mathrm{~Hz}), 0.91(\mathrm{~s}, 9 \mathrm{H}), 0.13$ $(\mathrm{s}, 3 \mathrm{H}), 0.11(\mathrm{~s}, 3 \mathrm{H}) ;{ }^{13} \mathrm{C} \mathrm{NMR}\left(75.0 \mathrm{MHz}, \mathrm{CDCl}_{3}\right) \delta 194.4,147.2,135.0,130.1,121.6$, 65.0, 63.7, 55.4, 53.3, 25.7, 19.1, 18.0, -5.7, -5.8; IR (thin film) vmax 3401, 3020, 2930, $2857,1682,1255,1216,1079,836,756,670 \mathrm{~cm}^{-1}$; CIHRMS M $\mathrm{M}^{+}$calculated for $\mathrm{C}_{16} \mathrm{H}_{26} \mathrm{O}_{4} \mathrm{Si}: 310.1600$, found: $310.1615 ;[\alpha]_{\mathrm{D}}^{22}=+171^{\circ}\left(\mathrm{c}=1.0, \mathrm{CHCl}_{3}\right)$.

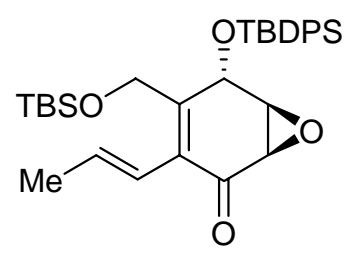

67

Bis-silyl ether 67: Silyl ether 37 (39 mg, $0.126 \mathrm{mmol})$ in 0.5 $\mathrm{mL}$ of DMF was added $69 \mathrm{mg}(0.251 \mathrm{mmol})$ of TBDPSCl and 34 $\mathrm{mg}(0.5 \mathrm{mmol})$ of imidazole. The reaction was stirred at $\mathrm{rt}$ for 4 $\mathrm{h}$, then $1 \mathrm{~mL}$ of water was added and the mixture extracted with EtOAc. The organic extracts were combined, washed with brine, dried over $\mathrm{MgSO}_{4}$, filtered, and concentrated in vacuo. Purification on silica gel (5\% EtOAc in hexane) provided $56 \mathrm{mg}$ $(0.102 \mathrm{mmol}, 81 \%)$ of $\mathbf{6 7}$ as a colorless oil. ${ }^{1} \mathrm{H}$ NMR (400 $\left.\mathrm{MHz}, \mathrm{CDCl}_{3}\right) \delta 7.67(\mathrm{~m}, 4 \mathrm{H}), 7.46-7.36(\mathrm{~m}, 6 \mathrm{H}), 5.99(\mathrm{~m}, 2 \mathrm{H}), 5.11(\mathrm{~s}, 1 \mathrm{H}), 4.47(\mathrm{~d}, 1 \mathrm{H}$, $J=12.8 \mathrm{~Hz}), 4.14(\mathrm{~d}, 1 \mathrm{H}, J=12.8 \mathrm{~Hz}), 3.48(\mathrm{dd}, 1 \mathrm{H}, J=2.0,3.6 \mathrm{~Hz}), 3.35(\mathrm{~d}, 1 \mathrm{H}, J=$ $3.6 \mathrm{~Hz}), 1.83(\mathrm{~d}, 3 \mathrm{H}, J=5.6 \mathrm{~Hz}), 1.00(\mathrm{~s}, 9 \mathrm{H}), 0.78(\mathrm{~s}, 9 \mathrm{H}),-0.08(\mathrm{~s}, 6 \mathrm{H}) ;{ }^{13} \mathrm{C} \mathrm{NMR}$ $\left(75.0 \mathrm{MHz}, \mathrm{CDCl}_{3}\right) \delta 195.9,147.8,135.8,134.8,133.3,132.7,130.2,128.0,122.2,64.6$, $59.9,55.6,53.3,26.6,25.6,19.3,19.0,-5.5,-5.6$; IR (thin film) vmax 3020, 2929, 2857, $1685,1469,1428,1255,1216,1111,1075,835,745,703 \mathrm{~cm}^{-1}$; CIHRMS [M+H] ${ }^{+}$ calculated for $\mathrm{C}_{32} \mathrm{H}_{45} \mathrm{O}_{4} \mathrm{Si}_{2}: 549.2858$, found: $549.2889 ;[\alpha]_{\mathrm{D}}{ }^{22}=+60.4^{\circ}\left(\mathrm{c}=0.5, \mathrm{CHCl}_{3}\right)$.

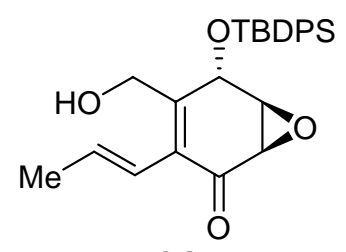

38

Primary alcohol 38: Compound 67 (50 $\mathrm{mg}, 0.091 \mathrm{mmol})$ was dissolved in $3 \mathrm{~mL}$ of $\mathrm{CH}_{3} \mathrm{CN}$ and $0.3 \mathrm{~mL}$ of $48 \%$ aqueous $\mathrm{HF}$ was added. The mixture was stirred at rt for $20 \mathrm{~min}$ before being quenched with sat. aqueous $\mathrm{NaHCO}_{3}$. The mixture was extracted with EtOAc, the organic extracts combined, washed with $5 \%$ $\mathrm{NaHCO}_{3}$ and brine, dried over $\mathrm{MgSO}_{4}$, filtered, and concentrated in vacuo. Purification on silica gel (10\% EtOAc in hexane) provided $31 \mathrm{mg}(0.071 \mathrm{mmol}, 78 \%)$ of $\mathbf{3 8}$ as a colorless oil. ${ }^{1} \mathrm{H}$ NMR (400 MHz, $\left.\mathrm{CDCl}_{3}\right) \delta 7.68(\mathrm{~m}, 4 \mathrm{H}), 7.48-7.39(\mathrm{~m}, 6 \mathrm{H}), 6.01(\mathrm{~m}, 2 \mathrm{H}), 5.00(\mathrm{~s}, 1 \mathrm{H})$, $4.32(\mathrm{~d}, 1 \mathrm{H}, J=13.6 \mathrm{~Hz}), 4.12(\mathrm{~d}, 1 \mathrm{H}, J=13.6 \mathrm{~Hz}), 3.57(\mathrm{~d}, 1 \mathrm{H}, J=4.0 \mathrm{~Hz}), 3.40(\mathrm{~d}, 1 \mathrm{H}$, $J=4.0 \mathrm{~Hz}), 1.82(\mathrm{~d}, 3 \mathrm{H}, J=4.4 \mathrm{~Hz}), 1.01(\mathrm{~s}, 9 \mathrm{H}) ;{ }^{13} \mathrm{C} \mathrm{NMR}\left(75.0 \mathrm{MHz}, \mathrm{CDCl}_{3}\right) \delta$ 195.5, 146.7, 135.8, 135.7, 135.3, 132.7, 131.6, 130.4, 128.1, 121.8, 65.6, 60.4, 55.6, 53.4, 26.6, 19.3, 19.2; IR (thin film) vmax 3478, 3019, 2931, 2858, 2361, 2340, 1683, $1428,1338,1110,1072,745,704 \mathrm{~cm}^{-1}$; CIHRMS $[\mathrm{M}+\mathrm{H}]^{+}$calculated for $\mathrm{C}_{26} \mathrm{H}_{31} \mathrm{O}_{4} \mathrm{Si}$ : 435.1993, found: $435.1953 ;[\alpha]_{\mathrm{D}}^{22}=+100^{\circ}\left(\mathrm{c}=1.0, \mathrm{CHCl}_{3}\right)$. 
TBDPS 2H-pyran 39/39': Alcohol 38 (17 mg, 0.039 mmol) was dissolved in $1 \mathrm{~mL}$ of $\mathrm{CH}_{2} \mathrm{Cl}_{2}$ and $27 \mathrm{mg}(0.064 \mathrm{mmol})$ of DessMartin periodinane was added. The mixture was stirred at $\mathrm{rt}$ for 30 min until total conversion of $\mathbf{3 8}$ was observed (TLC). $10 \mathrm{~mL}$ of EtOAc was added and the mixture was washed with $2 \times 3 \mathrm{~mL}$ of 1:1 of sat. $\mathrm{Na}_{2} \mathrm{~S}_{2} \mathrm{O}_{3} /$ sat. $\mathrm{NaHCO}_{3}, 3 \mathrm{~mL}$ of water, and $3 \mathrm{~mL}$ brine. The organic extract was dried over $\mathrm{MgSO}_{4}$, filtered, and concentrated in vacuo to afford $17 \mathrm{mg}(0.039 \mathrm{mmol}, 100 \%)$ of 39/39' as a colorless oil which was characterized without further purification. Major diastereomer: ${ }^{1} \mathrm{H}$ NMR (400 $\left.\mathrm{MHz}, \mathrm{CDCl}_{3}\right) \delta$ 7.68-7.60 (m, 4H), 7.47-7.35 (m, 6H), $6.03(\mathrm{~d}, 1 \mathrm{H}, J=2.8 \mathrm{~Hz}), 5.80(\mathrm{~s}$, $1 \mathrm{H}), 4.62(\mathrm{~d}, 1 \mathrm{H}, J=2.8 \mathrm{~Hz}), 4.49(\mathrm{~m}, 1 \mathrm{H}), 3.63(\operatorname{app~t}, 1 \mathrm{H}, J=3.2 \mathrm{~Hz}), 3.46(\mathrm{~d}, 1 \mathrm{H}, J=$ $3.6 \mathrm{~Hz}), 1.41$ (d, 3H, $6.8 \mathrm{~Hz}), 1.00(\mathrm{~s}, 9 \mathrm{H}) ;{ }^{13} \mathrm{C} \mathrm{NMR}\left(75.0 \mathrm{MHz}, \mathrm{CDCl}_{3}\right) \delta 192.6$, $147.3,136.0,135.9,133.2,130.2,128.0,127.8,125.6,112.6,71.5,66.1,57.8,53.9,29.6$, 26.7, 19.7; IR (thin film) vmax 3019, 2930, 2857, 1699, 1587, 1428, 1342, 1276, 1215, 1112, $1053 \mathrm{~cm}^{-1}$; CIHRMS M $\mathrm{M}^{+}$calculated for $\mathrm{C}_{26} \mathrm{H}_{28} \mathrm{O}_{4} \mathrm{Si}$ : 432.1757, found:432.1733; $[\alpha]_{\mathrm{D}}^{22}=+35.5^{\mathrm{o}}\left(\mathrm{c}=1.0, \mathrm{CHCl}_{3}\right)$.

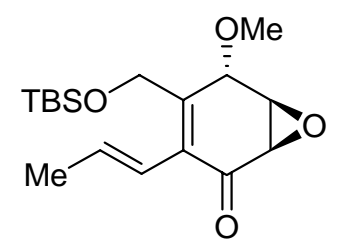

40

Methyl ether 40: Compound 37 (60 mg, $0.194 \mathrm{mmol}$ ), trimethyloxonium tetrafluoroborate $(142 \mathrm{mg}, 0.96 \mathrm{mmol})$ and 2,6-Di-tert-butyl-4-methylpyridine (264 mg, $1.29 \mathrm{mmol})$ in $2 \mathrm{~mL}$ of $\mathrm{CH}_{2} \mathrm{Cl}_{2}$ was stirred at $\mathrm{rt}$ for $3.5 \mathrm{~h}$. The solvent was reduced by argon flow before the crude mixture was subjected to silica gel chromatography $(10 \%$ EtOAc in hexane) to afford $61.7 \mathrm{mg}$ (0.190 mmol, $98 \%)$ of 40 as pale yellow oil. ${ }^{1} \mathrm{H}$ NMR (400 $\left.\mathrm{MHz}, \mathrm{CDCl}_{3}\right) \delta 5.94(\mathrm{~m}, 2 \mathrm{H}), 4.79(\mathrm{~s}, 1 \mathrm{H}), 4.49(\mathrm{~d}, 1 \mathrm{H}, J=13.6$ $\mathrm{Hz}), 4.34(\mathrm{~d}, 1 \mathrm{H}, J=13.6 \mathrm{~Hz}), 3.79(\mathrm{~d}, 1 \mathrm{H}, J=3.6 \mathrm{~Hz}), 3.51(\mathrm{~d}, 1 \mathrm{H}, J=3.6 \mathrm{~Hz}), 3.43(\mathrm{~s}$, $3 \mathrm{H}), 1.81(\mathrm{~d}, 3 \mathrm{H}, J=4.4 \mathrm{~Hz}), 0.89(\mathrm{~s}, 9 \mathrm{H}), 0.07(\mathrm{~s}, 3 \mathrm{H}), 0.06(\mathrm{~s}, 3 \mathrm{H}) ;{ }^{13} \mathrm{C}$ NMR $(75.0$ $\left.\mathrm{MHz}, \mathrm{CDCl}_{3}\right) \delta 194.8,146.4,135.0,131.5,121.7,70.5,60.4,56.8,54.0,53.4,25.8,19.1$, $-5.5,-5.6$; IR (thin film) vmax 2955, 2931, 2857, 1684, 1472, 1362, 1256, $1085 \mathrm{~cm}^{-1}$; CIHRMS $[\mathrm{M}+\mathrm{H}]^{+}$calculated for $\mathrm{C}_{17} \mathrm{H}_{29} \mathrm{O}_{4} \mathrm{Si}: 324.1837$, found: $324.1797 ; \quad[\alpha]_{\mathrm{D}}{ }^{22}=$ $+138^{\circ}\left(\mathrm{c}=1.0, \mathrm{CHCl}_{3}\right)$.

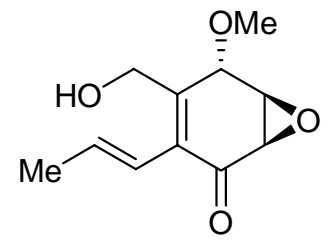

41

Primary alcohol 41: Compound 40 (60 mg, $0.185 \mathrm{mmol})$ was dissolved in $2 \mathrm{~mL}$ of $\mathrm{CH}_{3} \mathrm{CN}$ and $0.2 \mathrm{~mL}$ of $48 \%$ aqueous $\mathrm{HF}$ was added. The mixture was stirred at $\mathrm{rt}$ for $20 \mathrm{~min}$ before being quenched with water. The mixture was extracted with EtOAc, the organic extracts combined, washed with brine, dried over $\mathrm{Na}_{2} \mathrm{SO}_{4}$, filtered, and concentrated in vacuo. Purification on silica gel (30\% EtOAc in hexane) provided 38 $\mathrm{mg}(0.181 \mathrm{mmol}, 98 \%)$ of $\mathbf{4 1}$ as a pale yellow oil. ${ }^{1} \mathrm{H}$ NMR $\left(400 \mathrm{MHz}, \mathrm{CDCl}_{3}\right) \delta 6.01(\mathrm{~m}, 2 \mathrm{H}), 4.74(\mathrm{~s}, 1 \mathrm{H}), 4.42(\mathrm{~d}, 1 \mathrm{H}, J=14.0 \mathrm{~Hz}), 4.39(\mathrm{~d}, 1 \mathrm{H}, J$ $=14.0 \mathrm{~Hz}), 3.79(\mathrm{~d}, 1 \mathrm{H}, J=4.0 \mathrm{~Hz}), 3.53(\mathrm{~d}, 1 \mathrm{H}, J=4.0 \mathrm{~Hz}), 3.42(\mathrm{~s}, 3 \mathrm{H}), 1.80(\mathrm{~d}, 3 \mathrm{H}, J$ $=5.2 \mathrm{~Hz}) ;{ }^{13} \mathrm{C} \mathrm{NMR}\left(75.0 \mathrm{MHz}, \mathrm{CDCl}_{3}\right) \delta 194.6,144.8,135.5,132.5,121.4,100.0$, 72.1, 61.2, 53.9, 53.6, 19.1; IR (thin film) vmax 3446, 2935, 2829, 1683, 1653, 1456, 
1374, 1331, $1080 \mathrm{~cm}^{-1}$; CIHRMS $\mathrm{M}^{+}$calculated for $\mathrm{C}_{11} \mathrm{H}_{14} \mathrm{O}_{4}$ : 210.0892, found: $210.0884 ;[\alpha]_{\mathrm{D}}^{22}=+169^{\circ}\left(\mathrm{c}=1.0, \mathrm{CHCl}_{3}\right)$.

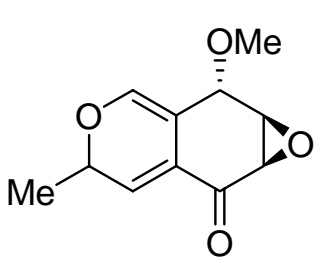

42/42'

2H-pyran 42/42': Alcohol 41 (27 mg, $0.129 \mathrm{mmol})$ was dissolved in $2 \mathrm{~mL}$ of $\mathrm{CH}_{2} \mathrm{Cl}_{2}$ at $0{ }^{\circ} \mathrm{C}$ and $58 \mathrm{mg}(0.137 \mathrm{mmol})$ of Dess-Martin periodinane was added. The mixture was stirred at 0 ${ }^{\circ} \mathrm{C}$ for $20 \mathrm{~min} .10 \mathrm{~mL}$ of EtOAc was added and the mixture was washed with $2 \times 3 \mathrm{~mL}$ of water, and $3 \mathrm{~mL}$ brine. The organic extract was dried over $\mathrm{Na}_{2} \mathrm{SO}_{4}$, filtered, and concentrated in vacuo. Purification on silica gel $\left(50 \%\right.$ EtOAc in $\left.\mathrm{CH}_{2} \mathrm{Cl}_{2}\right)$ provided $26 \mathrm{mg}(0.125 \mathrm{mmol}, 96 \%)$ of 42/42' as pale yellow oil. 42/42' exists as a mixture of two interchangeable diastereomeric $2 \mathrm{H}$-pyrans and small amount of corresponding aldehyde. Major $2 \mathrm{H}$-pyran diastereomer: ${ }^{1} \mathrm{H}$ NMR $(400 \mathrm{MHz}$, $\left.\mathrm{CDCl}_{3}\right) \delta 6.52(\mathrm{~s}, 1 \mathrm{H}), 6.09(\mathrm{~d}, 1 \mathrm{H}, J=3.2 \mathrm{~Hz}), 4.79(\mathrm{dq}, 1 \mathrm{H}, J=3.2,13.2 \mathrm{~Hz}), 4.16(\mathrm{~d}$, $1 \mathrm{H}, J=\quad 3.2 \mathrm{~Hz}), 3.73(\mathrm{~m}, 1 \mathrm{H}), 3.43(\mathrm{~d}, 1 \mathrm{H}, J=4.0 \mathrm{~Hz}), 3.26(\mathrm{~s}, 3 \mathrm{H}), 1.50(\mathrm{~d}, 3 \mathrm{H}, J$ $=13.2) ;{ }^{13} \mathrm{C} \mathrm{NMR}\left(75.0 \mathrm{MHz}, \mathrm{CDCl}_{3}\right) \delta 191.9,147.7,144.8,126.3,108.8,73.5,72.0$, 56.2, 55.4, 53.9, 19.9; IR (thin film) vmax 2927, 2854, 1699, 1593, 1457, 1339, 1277, 1155, $1084 \mathrm{~cm}^{-1}$; CIHRMS M${ }^{+}$calculated for $\mathrm{C}_{11} \mathrm{H}_{12} \mathrm{O}_{4}$ : 208.0736, found: 208.0736; $[\alpha]_{\mathrm{D}}^{22}=+166^{\circ}\left(\mathrm{c}=1.0, \mathrm{CHCl}_{3}\right)$.

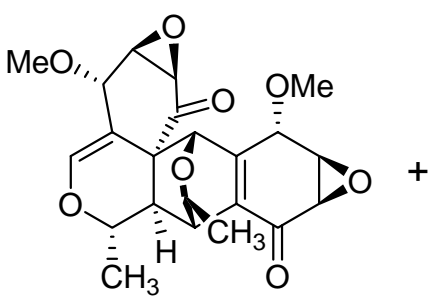

43

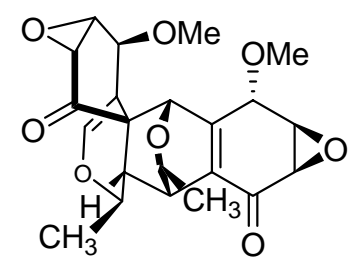

44

Dimethyl-epoxyquinols $A$ and $B$ 43 and 44: $18 \mathrm{mg}(0.0865 \mathrm{mmol})$ of 42/42' in $20 \mu \mathrm{L}$ of EtOAc was stirred at $\mathrm{rt}$ for $40 \mathrm{~h}$ in a round bottom flask covered by aluminum foil. The product mixture was purified on silica gel (30\% EtOAc in hexane) to provide $6.5 \mathrm{mg}$ $(0.0156 \mathrm{mmol}, 36 \%)$ of dimethylepoxyquinol A 43 and $4.8 \mathrm{mg}(0.0115,27 \%)$ of dimethyl-epoxyquinol B 44.

43: ${ }^{1} \mathrm{H}$ NMR $\left(400 \mathrm{MHz}, \mathrm{CDCl}_{3}\right) \delta 6.71(\mathrm{~d}, 1 \mathrm{H}, J=2.0 \mathrm{~Hz}), 5.14(\mathrm{~s}, 1 \mathrm{H}), 4.54(\mathrm{~d}, 1 \mathrm{H}, J=$ $2.0 \mathrm{~Hz}), 4.33(\mathrm{~m}, 3 \mathrm{H}), 3.74(\mathrm{~m}, 2 \mathrm{H}), 3.60(\mathrm{~s}, 3 \mathrm{H}), 3.54(\mathrm{~m}, 4 \mathrm{H}), 3.43(\mathrm{~m}, 3 \mathrm{H}), 3.10(\mathrm{~s}$, $1 \mathrm{H}), 2.51(\mathrm{~s}, 1 \mathrm{H}), 1.03(\mathrm{~d}, 3 \mathrm{H}, J=6.4 \mathrm{~Hz}), 0.78(\mathrm{~d}, 3 \mathrm{H}, J=6.0 \mathrm{~Hz}) ;{ }^{13} \mathrm{C} \mathrm{NMR} \quad(75.0$ $\left.\mathrm{MHz}_{\mathrm{CDCl}}\right) \delta 199.4,188.8,150.3,142.7,134.6,110.5,77.2,74.3,72.7,72.4,71.3$, $66.7,62.5,58.8,57.9,55.5,55.4,52.8,38.5,37.0,20.6,19.9$; IR (thin film) vmax 2930, 1683, 1456, 1201, $1088 \mathrm{~cm}^{-1}$; CIHRMS $[\mathrm{M}+\mathrm{H}]^{+}$calculated for $\mathrm{C}_{22} \mathrm{H}_{25} \mathrm{O}_{8}: 417.1551$, found: $417.1576 ;[\alpha]_{\mathrm{D}}^{22}=+81.3^{\circ}\left(\mathrm{c}=0.6, \mathrm{CHCl}_{3}\right)$.

44: ${ }^{1} \mathrm{H}$ NMR $\left(400 \mathrm{MHz}, \mathrm{CDCl}_{3}\right) \delta 6.46(\mathrm{~s}, 1 \mathrm{H}), 5.00(\mathrm{~s}, 1 \mathrm{H}), 4.54(\mathrm{~s}, 1 \mathrm{H}), 4.04(\mathrm{q}, 1 \mathrm{H}, J$ $=8.8 \mathrm{~Hz}), 3.84(\mathrm{dd}, 1 \mathrm{H}, J=1.2,3.6 \mathrm{~Hz}), 3.80(\mathrm{~s}, 1 \mathrm{H}), 3.63(\mathrm{dd}, 1 \mathrm{H}, J=1.6,3.6 \mathrm{~Hz}), 3.51$ $(\mathrm{m}, 2 \mathrm{H}), 3.44(\mathrm{~s}, 3 \mathrm{H}), 3.39(\mathrm{~s}, 3 \mathrm{H}), 3.20(\mathrm{dq}, 1 \mathrm{H}, J=8.8,13.6 \mathrm{~Hz}), 3.06(\mathrm{dd}, 1 \mathrm{H}, J=1.2$, $4.8 \mathrm{~Hz}), 2.86(\mathrm{dd}, 1 \mathrm{H}, J=4.8,13.6 \mathrm{~Hz}), 1.35(\mathrm{~d}, 3 \mathrm{H}, J=8.8 \mathrm{~Hz}), 0.72(\mathrm{~d}, 3 \mathrm{H}, J=8.8$ $\mathrm{Hz}) ;{ }^{13} \mathrm{C} \mathrm{NMR}\left(75.0 \mathrm{MHz}, \mathrm{CDCl}_{3}\right) \delta 199.0,191.4,150.8,146.7,134.9,103.0,73.9$, $72.6,72.2,68.6,57.8,56.5,54.7,53.6,52.8,52.6,52.3,39.9,36.1,29.6,20.0,18.8$; IR 
(thin film) vmax 2974, 2930, 2828, 1727, 1682, 1461, 1382, 1182, $1088 \mathrm{~cm}^{-1}$; CIHRMS $[\mathrm{M}+\mathrm{H}]^{+}$calculated for $\mathrm{C}_{22} \mathrm{H}_{25} \mathrm{O}_{8}: 417.1551$, found: 417.1539; $[\alpha]_{\mathrm{D}}{ }^{22}=+98^{\circ}(\mathrm{c}=0.43$, $\left.\mathrm{CHCl}_{3}\right)$.

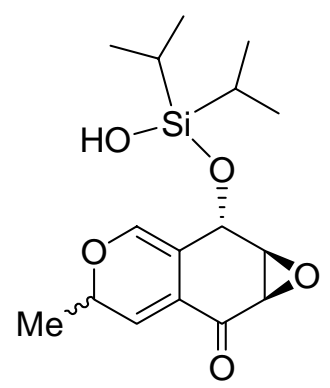

45/45'

Alkoxysilanol 2H-pyran 45/45': Monomer 9 (11 mg, 0.057 $\mathrm{mmol})$, TEMPO (2.6 mg, $0.018 \mathrm{mmol}, 0.3$ equiv) and $\mathrm{CuCl}(1.6$ $\mathrm{mg}, 0.016 \mathrm{mmol}, 0.28$ equiv) were dissolved in $0.5 \mathrm{~mL}$ of DMF and stirred at $\mathrm{rt}$ for $1 \mathrm{~h}$ under $\mathrm{O}_{2}(1 \mathrm{~atm})$ until full disappearance of starting material (TLC). After addition of $1 \mathrm{~mL}$ of sat. $\mathrm{CuSO}_{4}$, the reaction mixture was extracted with $3 \times 1 \mathrm{~mL}$ of EtOAc. The combined organic layers were washed with brine, dried over $\mathrm{MgSO}_{4}$, filtered, concentrated, and immediately subjected to silica gel chromatography ( $40 \%$ EtOAc in hexanes) to afford $2 \mathrm{H}$-pyran 10/10' as a yellowish eluant. $500 \mu \mathrm{L}$ of DMF was added and the solution was evaporated to a volume of $0.5 \mathrm{~mL}$. The residue was further evaporated under high vacuum for $5 \mathrm{~min}$, The resulting $2 \mathrm{H}$-pyran solution in DMF was added to a solution of $20 \mu \mathrm{L}$ of ${ }^{\mathrm{i}} \mathrm{Pr}_{2} \mathrm{SiCl}_{2}(0.11 \mathrm{mmol}, 2$ equiv) and $10 \mathrm{mg}$ imidazole $(0.15 \mathrm{mmol}, 2.5$ equiv) in $0.5 \mathrm{~mL} \mathrm{DMF}$ at $0^{\circ} \mathrm{C}$. The mixture was stirred for $15 \mathrm{~min}$ and quenched by addition of 2 $\mathrm{mL}$ of water. The mixture was extracted with EtOAc, the organic extracts were combined, washed with brine, dried over $\mathrm{MgSO}_{4}$, filtered, and concentrated in vacuo. Purification on silica gel (10\% EtOAc in hexane) provided $14 \mathrm{mg}(0.043 \mathrm{mmol}, 76 \%)$ of alkoxysilanol 45/45' as a colorless oil (1:1 mixture of $2 \mathrm{H}$-pyran diastereomers $(85 \%)$ and the corresponding aldehyde (15\%)). NMR data for the $2 \mathrm{H}$-pyrans: ${ }^{1} \mathrm{H}$ NMR (400 $\left.\mathrm{MHz}, \mathrm{CDCl}_{3}\right) \delta 6.56(\mathrm{~s}, 1 \mathrm{H}), 6.40(\mathrm{~s}, 1 \mathrm{H}), 6.18(\mathrm{~d}, 1 \mathrm{H}, J=6.8 \mathrm{~Hz}), 6.06(\mathrm{~d}, 1 \mathrm{H}, J=4.0$ $\mathrm{Hz}), 5.00(\mathrm{~m}, 1 \mathrm{H}), 4.91(\mathrm{~d}, 1 \mathrm{H}, J=4.0 \mathrm{~Hz}), 4.87(\mathrm{~d}, 1 \mathrm{H}, J=4.0 \mathrm{~Hz}), 4.74(\mathrm{~m}, 1 \mathrm{H}), 3.69$ (app q, 2H, $J=4.8 \mathrm{~Hz}$ ), 3.42 (app d, $2 \mathrm{H}, J=4.8 \mathrm{~Hz}$ ), 2.68 (br s, 1H, OH), 2.47 (br s, $1 \mathrm{H}$, $\mathrm{OH}), 1.48(\mathrm{~d}, 3 \mathrm{H}, J=8.8 \mathrm{~Hz}), 1.32(\mathrm{~d}, 3 \mathrm{H}, J=8.8 \mathrm{~Hz}), 0.99(\mathrm{~m}, 14 \mathrm{H}) ;{ }^{13} \mathrm{C}$ NMR $(75.0$ $\left.\mathrm{MHz}, \mathrm{CDCl}_{3}\right) \delta 193.9,192.6,147.1,144.5,143.6,140.4,125.6,125.5,113.5,111.1$, $71.8,71.2,64.9,58.3,58.0,54.0,53.9,19.8,18.8,16.9,16.8,12.9,12.5$; IR (thin film) vmax 3446, 2945, 2895, 2867, 1698, 1593, 1464, 1279, 1055, $1002 \mathrm{~cm}^{-1}$; CIHRMS M ${ }^{+}$ calculated for $\mathrm{C}_{16} \mathrm{H}_{24} \mathrm{O}_{5} \mathrm{Si}: 324.1393$, found: $324.1408 ;[\alpha]_{\mathrm{D}}{ }^{22}=+84^{\circ}\left(\mathrm{c}=1.0, \mathrm{CHCl}_{3}\right)$.

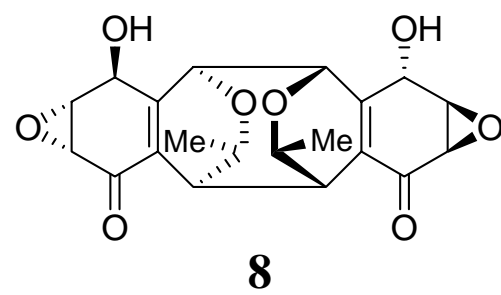

RKB-3564D (8): $22 \mathrm{mg}$ (0.068 mmol) of 45/45' was concentrated in a scintillation vial and kept at $\mathrm{rt}$ for $30 \mathrm{~h}$ until starting material disappeared (by ${ }^{1} \mathrm{H}$ NMR). The crude mixture was dissolved in $0.5 \mathrm{~mL}$ of THF and $20 \mu \mathrm{L}$ of $\mathrm{Et}_{3} \mathrm{~N} \cdot 3 \mathrm{HF}$ was added at $0^{\circ} \mathrm{C}$. The reaction mixture was stirred at $0^{\circ} \mathrm{C}$ for $25 \mathrm{~min}$, concentrated in vacuo, and purified by silica gel chromatography (30:30:1 of $\left.\mathrm{CH}_{2} \mathrm{Cl}_{2}: \mathrm{CHCl}_{3}: \mathrm{MeOH}\right)$ to afford $5.3 \mathrm{mg}(0.014 \mathrm{mmol}, 40 \%)$ of RKB-3564D (8). ${ }^{1} \mathrm{H}$ NMR and ${ }^{13} \mathrm{C}$ NMR (see page 4). IR (thin film) vmax 3398, 2941, 2833, 1708, $1675,1456,1374,1230,1032 \mathrm{~cm}^{-1}$; CIHRMS $[\mathrm{M}+\mathrm{H}]^{+}$calculated for $\mathrm{C}_{26} \mathrm{H}_{31} \mathrm{O}_{4} \mathrm{Si}$ : 388.1158, found: 388.1133 ; $[\alpha]_{\mathrm{D}}{ }^{22}=+293^{\circ}(\mathrm{c}=0.15$, acetone). Natural RKB-3564D: $[\alpha]_{\mathrm{D}}^{21}=+303.3^{\circ}(\mathrm{c}=0.184$, acetone $)$. 


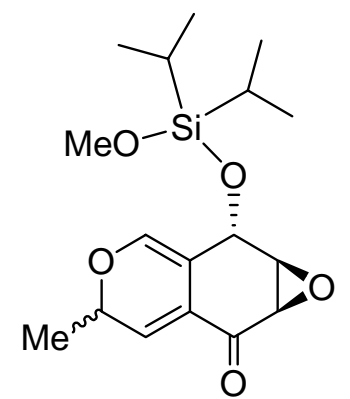

49/49'

Methoxy silane 49/49': 9 (36 mg, $0.184 \mathrm{mmol}$ ), TEMPO (9 mg, $0.057 \mathrm{mmol}, 0.3$ equiv) and $\mathrm{CuCl}$ ( $9 \mathrm{mg}, 0.09 \mathrm{mmol}, 0.5$ equiv) were dissolved in $1 \mathrm{~mL}$ of DMF and stirred at $\mathrm{rt}$ for $0.5 \mathrm{~h}$ under $\mathrm{O}_{2}$ (1 atm) until full disappearance of starting material (TLC). After addition of $2 \mathrm{~mL}$ of sat. $\mathrm{CuSO}_{4}$, the reaction mixture was extracted with $4 \times 3 \mathrm{~mL}$ of EtOAc. The combined organic layers were washed with brine, dried over $\mathrm{MgSO}_{4}$, filtered, concentrated, and immediately subjected to silica gel chromatography (40\% EtOAc in hexanes) to afford $2 \mathrm{H}$-pyran 10/10' as a yellowish eluant. $500 \mu \mathrm{L}$ of DMF was added and the solution was evaporated to a volume of $0.5 \mathrm{~mL}$. The residue was further evaporated under high vacuum for $5 \mathrm{~min}$, The resulting $2 \mathrm{H}$-pyran solution in DMF was added to a solution of $51 \mu \mathrm{L}$ of ${ }^{\mathrm{i}} \mathrm{Pr}_{2} \mathrm{SiCl}_{2}(0.27 \mathrm{mmol}, 1.5$ equiv) and $44 \mathrm{mg}$ imidazole $\left(0.65 \mathrm{mmol}, 3.5\right.$ equiv) in $1 \mathrm{~mL}$ DMF at $0^{\circ} \mathrm{C}$. The mixture was stirred for 15 min and then $1 \mathrm{ml}$ of $\mathrm{MeOH}$ was added and stirred for $15 \mathrm{~min}$ at $0^{\circ} \mathrm{C}$. The reaction was quenched by addition of $2 \mathrm{~mL}$ of water. The mixture was extracted with EtOAc, the organic extracts were combined, washed with brine, dried over $\mathrm{MgSO}_{4}$, filtered, and concentrated in vacuo. Purification on silica gel (10\% EtOAc in hexane) provided $20 \mathrm{mg}$ (0.059 mmol, $32.2 \%$ ) of 49/49' as a pale yellow oil (1.5:1 mixture of $2 \mathrm{H}$-pyran diastereomers), $12 \mathrm{mg}$ (0.037 mmol, $20 \%)$ of 46/46' and $10 \mathrm{mg}(0.02 \mathrm{mmol}, 22 \%)$ of 50.

49/49': ${ }^{1} \mathrm{H}$ NMR (400 MHz, $\left.\mathrm{CDCl}_{3}\right) \delta 6.56(\mathrm{~s}, 1 \mathrm{H}), 6.40(\mathrm{~s}, 1 \mathrm{H}), 6.19(\mathrm{~d}, 1 \mathrm{H}, J=4.8$ $\mathrm{Hz}), 6.06(\mathrm{~d}, 1 \mathrm{H}, J=2.8 \mathrm{~Hz}), 5.00(\mathrm{~m}, 1 \mathrm{H}), 4.86(\mathrm{~d}, 1 \mathrm{H}, J=2.4 \mathrm{~Hz}), 4.82(\mathrm{~d}, 1 \mathrm{H}, J=3.2$ $\mathrm{Hz}), 4.75$ (m, 1H), 3.70 (app q, 2H, $J=4.0 \mathrm{~Hz}), 3.54$ (s, 6H), $3.43(\mathrm{~m}, 2 \mathrm{H}), 1.49$ (d, 3H, $J$ $=6.4 \mathrm{~Hz}), 1.32(\mathrm{~d}, 3 \mathrm{H}, J=6.4 \mathrm{~Hz}), 1.00(\mathrm{~m}, 14 \mathrm{H}) ;{ }^{13} \mathrm{C} \mathrm{NMR}\left(75.0 \mathrm{MHz}, \mathrm{CDCl}_{3}\right) \delta$ $192.9,192.4,147.1,144.5,129.5,127.1,125.4,120.3,113.3,110.9,71.8,71.2,65.1$, $58.2,58.0,54.1,54.0,51.2,50.7,19.9,18.9,17.1,17.0,13.1,12.6,12.1$; IR (thin film) vmax 2945, 2867, 1701, 1595, 1464, 1277, 1086, $1055 \mathrm{~cm}^{-1}$; CIHRMS M M $^{+}$calculated for $\mathrm{C}_{17} \mathrm{H}_{26} \mathrm{O}_{5} \mathrm{Si}: 338.1550$, found: $338.1536 ;[\alpha]_{\mathrm{D}}{ }^{22}=+38.6^{\mathrm{o}}\left(\mathrm{c}=1.0, \mathrm{CHCl}_{3}\right)$.

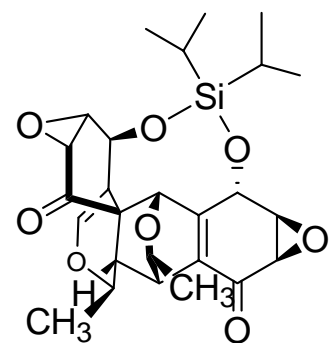

50

Dialkoxysilane 50: To epoxyquinol B (16 mg, $0.041 \mathrm{mmol})$ in $0.8 \mathrm{~mL}$ of DMF was added $15 \mathrm{mg}(0.081 \mathrm{mmol})$ of ${ }^{\mathrm{i}} \mathrm{Pr}_{2} \mathrm{SiCl}_{2}$ and $9.8 \mathrm{mg}(0.144 \mathrm{mmol})$ of imidazole. The reaction was stirred at $\mathrm{rt}$ for $3 \mathrm{~h}, 1 \mathrm{~mL}$ of water was added, and the mixture extracted with EtOAc. The organic extracts were combined, washed with brine, dried over $\mathrm{MgSO}_{4}$, filtered, and concentrated in vacuo. Purification on silica gel (20\% EtOAc in hexane) provided 20.5 $\mathrm{mg}(0.041 \mathrm{mmol}, 100 \%)$ of $\mathbf{5 0}$ as a white solid. $\mathrm{mp} 233-235^{\circ} \mathrm{C}$.

${ }^{1} \mathrm{H}$ NMR $\left(400 \mathrm{MHz}, \mathrm{CDCl}_{3}\right) \delta 6.39(\mathrm{~s}, 1 \mathrm{H}), 5.02(\mathrm{~s}, 1 \mathrm{H}), 4.94$ $(\mathrm{s}, 1 \mathrm{H}), 4.70(\mathrm{~d}, 1 \mathrm{H}, J=2.4 \mathrm{~Hz}), 4.14(\mathrm{q}, 1 \mathrm{H}, J=6.4 \mathrm{~Hz}), 3.72$ $(\mathrm{d}, 1 \mathrm{H}, J=3.2 \mathrm{~Hz}), 3.61(\mathrm{t}, 1 \mathrm{H}, J=2.8 \mathrm{~Hz}), 3.55(\mathrm{q}, 1 \mathrm{H}, J=6.4 \mathrm{~Hz}), 3.50(\mathrm{~d}, 1 \mathrm{H}, J=3.2$ $\mathrm{Hz}), 3.43(\mathrm{~d}, 1 \mathrm{H}, J=3.2 \mathrm{~Hz}), 3.07(\mathrm{~d}, 1 \mathrm{H}, J=1.6 \mathrm{~Hz}), 2.71(\mathrm{dd}, 1 \mathrm{H}, J=2.8,6.0 \mathrm{~Hz})$, $1.22(\mathrm{~d}, 3 \mathrm{H}, J=6.4 \mathrm{~Hz}), 1.20-1.00(\mathrm{~m}, 14 \mathrm{H}), 0.78(\mathrm{~d}, 3 \mathrm{H}, J=6.4 \mathrm{~Hz}) ;{ }^{13} \mathrm{C} \mathrm{NMR}(75.0$ $\left.\mathrm{MHz} \mathrm{CDCl}_{3}\right) \delta 199.3,190.7,149.9,148.7,132.3,106.5,74.2,72.9,71.2,69.1,64.3$, 
$57.2,54.8,52.6,52.3,42.0,36.6,29.5,19.8,19.2,17.7,17.4,17.0,16.9,12.9,11.6$; IR (thin film) vmax 2945, 2867, 1716, 1684, 1464, 1086, 1051, $886 \mathrm{~cm}^{-1}$; CIHRMS M calculated for $\mathrm{C}_{26} \mathrm{H}_{32} \mathrm{O}_{8} \mathrm{Si}$ : 500.1866, found: $500.1895 ;[\alpha]_{\mathrm{D}}{ }^{22}=+162^{\circ}\left(\mathrm{c}=1.0, \mathrm{CHCl}_{3}\right.$ ).

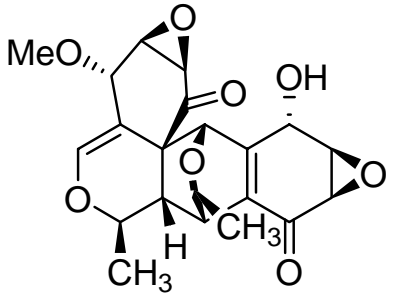

53

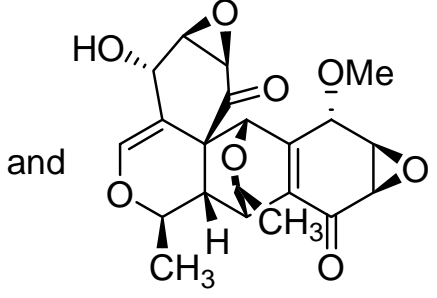

54

Monomethyl epoxyquinol B 53 and 54: Epoxyquinol B 7 (30 $\mathrm{mg}, \quad 0.077 \mathrm{mmol})$, trimethyloxonium tetrafluoroborate (53 $\mathrm{mg}, 0.358 \mathrm{mmol}$ ) and 2,6-Di-tertbutyl-4-methylpyridine (92 $\mathrm{mg}$, $0.449 \mathrm{mmol}$ ) in $1 \mathrm{~mL}$ of $\mathrm{CH}_{2} \mathrm{Cl}_{2}$ were stirred at $\mathrm{rt}$ for $4 \mathrm{~h}$. The solvent was reduced by argon flow before the crude mixture was subjected to silica gel chromatography (4\% EtOAc in $\mathrm{CH}_{2} \mathrm{Cl}_{2}$ ) to afford $10.3 \mathrm{mg}$ $(0.026 \mathrm{mmol}, 33 \%)$ of 53 and $19.3 \mathrm{mg}(0.048 \mathrm{mmol}, 62 \%)$ of 54.

53: ${ }^{1} \mathrm{H}$ NMR $\left(400 \mathrm{MHz}, \mathrm{CDCl}_{3}\right) \delta 6.58(\mathrm{~s}, 1 \mathrm{H}), 5.08(\mathrm{~s}, 1 \mathrm{H}), 4.86(\mathrm{~s}, 1 \mathrm{H}), 4.74(\mathrm{~s}, 1 \mathrm{H})$, 4.10 (q, $1 \mathrm{H}, J=6.4 \mathrm{~Hz}), 3.80$ (d, $1 \mathrm{H}, J=3.2 \mathrm{~Hz}), 3.71$ (d, $1 \mathrm{H}, J=1.6 \mathrm{~Hz}), 3.69$ (s, 3H), 3.49 (app t, $2 \mathrm{H}, J=3.2 \mathrm{~Hz}), 3.26(\mathrm{dq}, 1 \mathrm{H}, J=8.8,6.4 \mathrm{~Hz}), 3.08(\mathrm{~d}, 1 \mathrm{H}, J=3.6 \mathrm{~Hz}), 2.84$ $(\mathrm{dd}, 1 \mathrm{H}, J=3.6,8.8 \mathrm{~Hz}), 1.31(\mathrm{~d}, 3 \mathrm{H}, J=6.4 \mathrm{~Hz}), 0.76(\mathrm{~d}, 3 \mathrm{H}, J=6.4 \mathrm{~Hz}) ;{ }^{13} \mathrm{C} \mathrm{NMR}$ $\left(75.0 \mathrm{MHz}, \mathrm{CDCl}_{3}\right) \delta 198.0,191.0,152.4,150.6,132.2,103.4,78.2,77.2,74.5,72.7$, $69.7,63.3,58.1,56.1,52.6,52.3,50.9,40.9,35.8,20.2,19.1$; IR (thin film) vmax 3370, 2966, 2930, 1730, 1685, 1653, 1261, $1057 \mathrm{~cm}^{-1}$; CIHRMS M $\mathrm{M}^{+}$calculated for $\mathrm{C}_{21} \mathrm{H}_{22} \mathrm{O}_{8}$ : 403.1395, found: $403.1361 ;[\alpha]_{\mathrm{D}}^{22}=+108.4^{\circ}\left(\mathrm{c}=0.5, \mathrm{CHCl}_{3}\right)$.

54: ${ }^{1} \mathrm{H}$ NMR $\left(400 \mathrm{MHz}, \mathrm{CDCl}_{3}\right) \delta 6.41(\mathrm{~s}, 1 \mathrm{H}), 5.10(\mathrm{~s}, 1 \mathrm{H}), 4.66(\mathrm{~s}, 1 \mathrm{H}), 4.51(\mathrm{~d}, 1 \mathrm{H}, J$ $=2.0 \mathrm{~Hz}), 4.27(\mathrm{~s}, 1 \mathrm{H}), 4.15(\mathrm{q}, 1 \mathrm{H}, J=6.4 \mathrm{~Hz}), 3.87(\mathrm{~d}, 1 \mathrm{H}, J=4.0 \mathrm{~Hz}), 3.65(\mathrm{dd}, 1 \mathrm{H}, J$ $=2.4,3.2 \mathrm{~Hz}), 3.55(\mathrm{~m}, 2 \mathrm{H}), 3.52(\mathrm{~s}, 3 \mathrm{H}), 3.44(\mathrm{~d}, 1 \mathrm{H}, J=2.8 \mathrm{~Hz}), 3.10(\mathrm{~d}, 1 \mathrm{H}, J=2.4$ $\mathrm{Hz}), 2.74(\mathrm{dd}, 1 \mathrm{H}, J=2.4,6.0 \mathrm{~Hz}), 1.24(\mathrm{~d}, 3 \mathrm{H}, J=6.4 \mathrm{~Hz}), 0.76(\mathrm{~d}, 3 \mathrm{H}, J=6.4 \mathrm{~Hz})$; ${ }^{13} \mathrm{C}$ NMR $\left(75.0 \mathrm{MHz}, \mathrm{CDCl}_{3}\right) \delta 199.3,190.0,148.6,146.2,135.1,107.0,74.2,73.0$, $72.3,70.6,67.5,55.8,54.7,54.1,52.7,52.5,50.9,41.9,37.2,19.9,19.5$; IR (thin film) vmax 3402, 2973, 2926, 1685, 1653, 1191, $1060 \mathrm{~cm}^{-1}$; CIHRMS M calculated for $\mathrm{C}_{21} \mathrm{H}_{22} \mathrm{O}_{8}$ : 403.1395, found: 403.1373; $[\alpha]_{\mathrm{D}}{ }^{22}=+149^{\circ}\left(\mathrm{c}=1.0, \mathrm{CHCl}_{3}\right)$. 


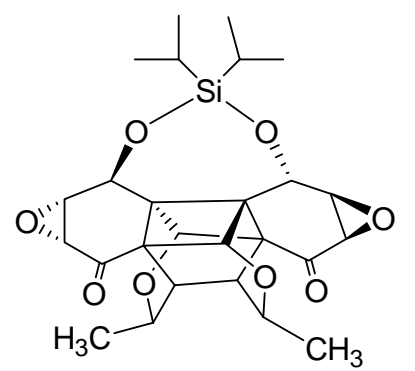

55

Photoproduct 55: Dialkoxysilane 50 (9 $\mathrm{mg}, 0.018 \mathrm{mmol}$ ) was dissolved in $1 \mathrm{~mL}$ of degassed THF and transferred to a NMR tube under an Ar atmosphere. The solution was photolyzed using a Pyrex filter for 40 min until the starting material was consumed (TLC). The solution was concentrated in vacuo and purified on silica gel chromatography (20\% EtOAc in hexane) provided $4.5 \mathrm{mg}$ $(0.009 \mathrm{mmol}, 50 \%)$ of 55 as a yellowish solid. mp 235-237 ${ }^{\circ} \mathrm{C}{ }^{1} \mathrm{H}$ NMR $\left(400 \mathrm{MHz}, \mathrm{CDCl}_{3}\right) \delta 4.61(\mathrm{~d}, 2 \mathrm{H}, J=3.2 \mathrm{~Hz})$, 4.26 (s, 2H), 4.25 (q, 2H, $J=8.8 \mathrm{~Hz}), 3.66$ (dd, $2 \mathrm{H}, J=3.2$, $4.8 \mathrm{~Hz}), 3.39$ (d, 2H, $J=4.8 \mathrm{~Hz}), 2.23(\mathrm{~s}, 2 \mathrm{H}), 1.32(\mathrm{~d}, 2 \mathrm{H}, J$ $=8.8 \mathrm{~Hz}), 1.12-1.00(\mathrm{~m}, 14 \mathrm{H}) ;{ }^{13} \mathrm{C} \mathrm{NMR}\left(75.0 \mathrm{MHz}, \mathrm{CDCl}_{3}\right) \delta 205.1,78.1,73.7,66.2$, 59.9, 52.7, 43.4, 29.6, 19.7, 17.4, 16.7, 11.2; IR (thin film) vmax 3020, 2926, 2855, 1699, $1559,1457,1216,1042 \mathrm{~cm}^{-1}$; CIHRMS M${ }^{+}$calculated for $\mathrm{C}_{26} \mathrm{H}_{32} \mathrm{O}_{8} \mathrm{Si}: 500.1866$, found: $500.1890 ;[\alpha]_{\mathrm{D}}^{22}=+60^{\circ}\left(\mathrm{c}=0.5, \mathrm{CHCl}_{3}\right)$.

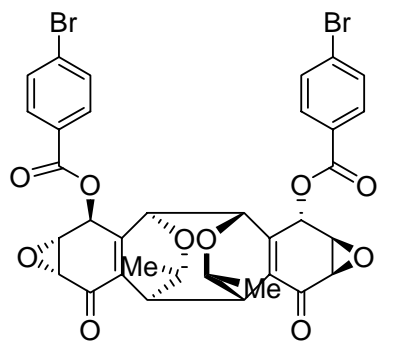

57

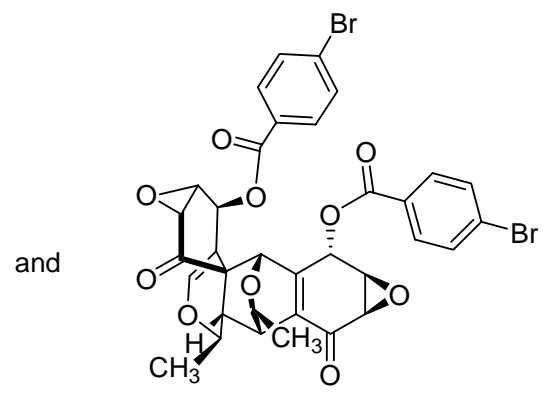

58
Bis-bromobenzoates 57 and 58: $4.5 \mathrm{mg}(0.012$ $\mathrm{mmol})$ of $\mathbf{8}$ and $10 \mathrm{mg}$ (0.046 mmol, 3.9 equiv.) of 4-bromobenzoyl chloride were dissolved in $0.5 \mathrm{~mL}$ of $\mathrm{CH}_{2} \mathrm{Cl}_{2}$, to which $0.4 \mathrm{mg}$ of DMAP ( 0.003 mmol, 0.3 equiv) and $\quad 10 \mu \mathrm{L} \quad$ of

triethylamine $\left(0.072 \mathrm{mmol}, 6\right.$ equiv.) were added at $0^{\circ} \mathrm{C}$. After stirring at for $60 \mathrm{~min}$, volatiles were removed by an argon stream and the residue was subjected to flash chromatography (15:15:1 hexane: $\mathrm{CH}_{2} \mathrm{Cl}_{2}$ :EtOAc) to afford $8.1 \mathrm{mg}(0.011 \mathrm{mmol}, 93 \%)$ of bis-p-bromobenzoate 57 as a white solid. ${ }^{1} \mathrm{H}$ NMR $\left(400 \mathrm{MHz}, \mathrm{CDCl}_{3}\right) \delta 7.87(\mathrm{~d}, 4 \mathrm{H}, J=$ $8.8 \mathrm{~Hz}), 7.58(\mathrm{~d}, 4 \mathrm{H}, J=8.8 \mathrm{~Hz}), 5.82(\mathrm{~d}, 2 \mathrm{H}, J=1.6 \mathrm{~Hz}), 4.68(\mathrm{~s}, 2 \mathrm{H}), 4.10(\mathrm{q}, 2 \mathrm{H}, J=$ $8.4 \mathrm{~Hz}$ ), 3.91 (dd, 2H, $J=1.6,4.8 \mathrm{~Hz}), 363$ (d, 2H, $J=4.8 \mathrm{~Hz}), 3.27$ (s, 2H), 0.69 (d, 6H, $J=8.4 \mathrm{~Hz}) ;{ }^{13} \mathrm{C} \mathrm{NMR}\left(75.0 \mathrm{MHz}, \mathrm{CDCl}_{3}\right) \delta 192.2,165.4,135.2,132.0,131.3,128.9$, 128.3, 81.1, 72.1, 67.8, 54.0, 52.2, 39.4, 22.9; IR (thin film) vmax 2926, 2853, 1724, 1683, 1590, 1398, 1259, 1096, $1011 \mathrm{~cm}^{-1}$; CIHRMS $[\mathrm{M}+\mathrm{H}]^{+}$calculated for $\mathrm{C}_{34} \mathrm{H}_{26} \mathrm{Br}_{2} \mathrm{O}_{10}$ : 751.9893, found: $751.9866 ;[\alpha]_{\mathrm{D}}{ }^{22}=+80^{\circ}\left(\mathrm{c}=0.5, \mathrm{CHCl}_{3}\right) .8 .1 \mathrm{mg}$ of 57 in $1 \mathrm{~mL}$ of $\mathrm{CDCl}_{3}$ was allowed to stand at $\mathrm{rt}$ for $2 \mathrm{~d}$ until total conversion to $\mathbf{5 8}$ was observed ( ${ }^{1} \mathrm{H}$ NMR). The solvent was concentrated and the residue subjected to flash chromatography $\left(5: 2: 1\right.$ hexane: $\mathrm{CH}_{2} \mathrm{Cl}_{2}$ :EtOAc) to afford $7 \mathrm{mg}(86 \%)$ of 58 as white solid. mp 224-226 ${ }^{\circ} \mathrm{C} .{ }^{1} \mathrm{H}$ NMR (400 MHz, $\left.\mathrm{CDCl}_{3}\right) \delta 7.62(\mathrm{~d}, 2 \mathrm{H}, J=11.6 \mathrm{~Hz}), 7.58(\mathrm{~d}$, $2 \mathrm{H}, J=11.6 \mathrm{~Hz}), 7.48(\mathrm{~d}, 2 \mathrm{H}, J=11.6 \mathrm{~Hz}), 7.37(\mathrm{~d}, 2 \mathrm{H}, J=11.6 \mathrm{~Hz}), 6.53(\mathrm{~s}, 1 \mathrm{H}), 6.01$ $(\mathrm{s}, 1 \mathrm{H}), 5.75(\mathrm{~d}, 1 \mathrm{H}, J=4.0 \mathrm{~Hz}), 4.96(\mathrm{~s}, 1 \mathrm{H}), 4.36(\mathrm{q}, 1 \mathrm{H}, J=8.4 \mathrm{~Hz}), 3.96(\mathrm{~m}, 1 \mathrm{H}), 3.78$ $(\mathrm{dd}, 1 \mathrm{H}, J=2.0,4.8 \mathrm{~Hz}), 3.58(\operatorname{app} \mathrm{t}, 1 \mathrm{H}, J=4.0 \mathrm{~Hz}), 3.50(\mathrm{~d}, 1 \mathrm{H}, J=4.8 \mathrm{~Hz}), 3.43$ (d, $1 \mathrm{H}, J=4.4 \mathrm{~Hz}), 3.19(\mathrm{~s}, 1 \mathrm{H}), 2.60(\mathrm{~d}, 1 \mathrm{H}, J=4.0 \mathrm{~Hz}), 1.28(\mathrm{~d}, 3 \mathrm{H}, J=8.8 \mathrm{~Hz}), 0.88(\mathrm{~d}$, $3 \mathrm{H}, J=8.4 \mathrm{~Hz}) ;{ }^{13} \mathrm{C} \mathrm{NMR}\left(75.0 \mathrm{MHz}, \mathrm{CDCl}_{3}\right) \delta 200.3,190.9,164.6,164.3,149.7$, 
$144.8,136.8,132.2,132.0,131.0,129.4,127.7,127.2,103.9,74.6,74.0,70.6,66.3,53.7$, 53.0, 52.1, 49.6, 45.1, 38.4, 29.6, 19.8, 19.6; IR (thin film) vmax 3020, 2926, 2854, 1725, $1686,1590,1261,1216,1093,1011 \mathrm{~cm}^{-1}$; CIHRMS $[\mathrm{M}+\mathrm{H}]^{+}$calculated for $\mathrm{C}_{34} \mathrm{H}_{26} \mathrm{Br}_{2} \mathrm{O}_{10}$ : 751.9893, found: $751.9907 ;[\alpha]_{\mathrm{D}}^{22}=-80^{\circ}\left(\mathrm{c}=0.23, \mathrm{CHCl}_{3}\right)$.
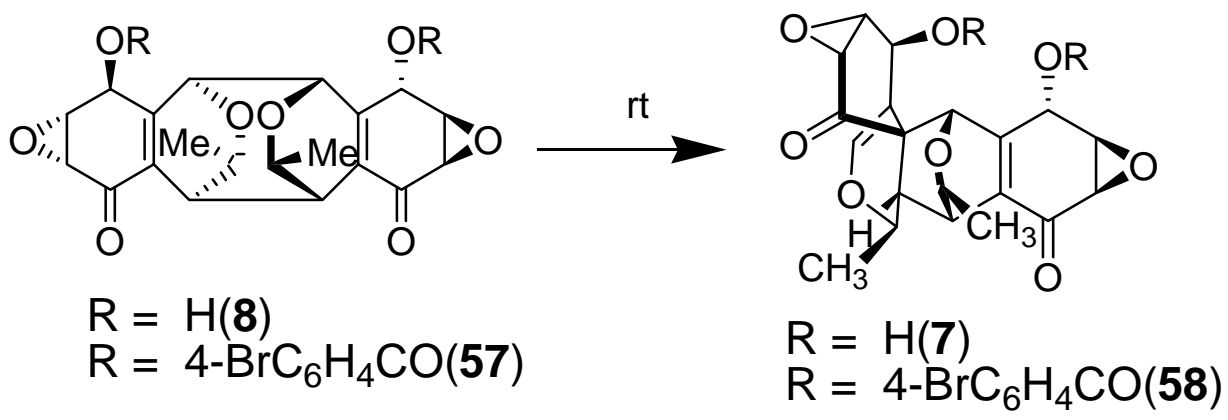

Experimental conditions for transformation of $8 \rightarrow \mathbf{7}$ and $\mathbf{5 7} \rightarrow \mathbf{5 8 :} 0.5 \mathrm{mg}$ of 8 or $\mathbf{5 7}$ was dissolved in $150 \mu \mathrm{L}$ solvent $(8.6 \mathrm{mM})$, the solutions were kept at $\mathrm{rt}$ in the dark for 14 $\mathrm{h},{ }^{1} \mathrm{H}$ NMR was used to determine the conversion (see table) of $\mathbf{8} \rightarrow \mathbf{7}$ or $\mathbf{5 7} \rightarrow \mathbf{5 8}$ after solvent removal.

\begin{tabular}{|c|c|c|c|}
\hline Conversion after $14 \mathrm{~h}\left({ }^{1} \mathrm{H}\right.$ NMR $)$ & in $\mathrm{PhCH}_{3}$ & in EtOAc & in $\mathrm{MeOH}$ \\
\hline $\mathbf{8} \rightarrow \mathbf{7}$ & $<5 \%$ & $21 \%$ & $59 \%$ \\
\hline $\mathbf{5 7} \rightarrow \mathbf{5 8}$ & $\mathrm{ND}$ & $53 \%$ & ND \\
\hline
\end{tabular}

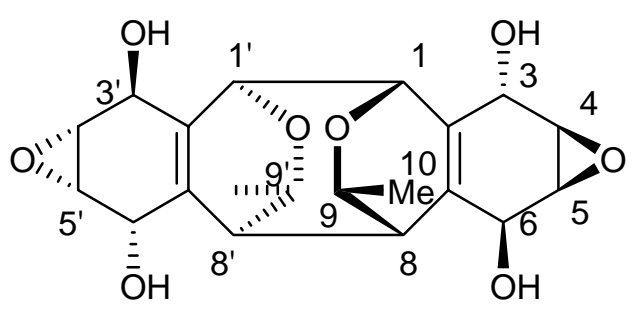

59

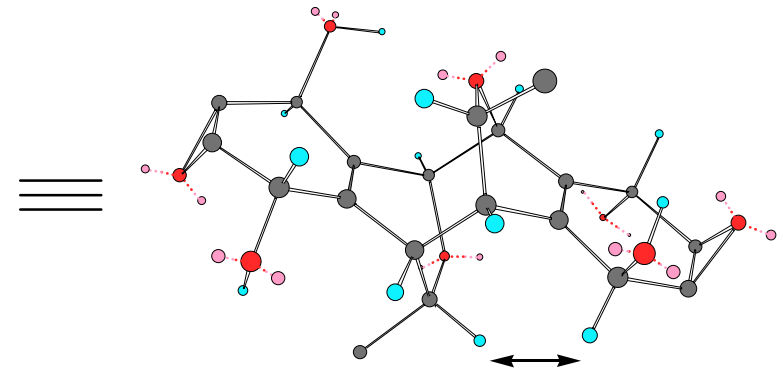

$4 \%$ NOE

Tetraol 59: To $2.3 \mathrm{mg}(0.006 \mathrm{mmol})$ of RKB-3564 D in $1 \mathrm{~mL}$ of THF was added 18 (L $\left(0.018 \mathrm{mmol}, 3\right.$ equiv. ) of $1.0 \mathrm{M}$ of Super-H in THF at $-50{ }^{\circ} \mathrm{C}$. The mixture was warmed to $-30^{\circ} \mathrm{C}$ over $30 \mathrm{~min}$ and the reaction was quenched by addition of $0.6 \mathrm{~mL}$ of $1: 1$ $\mathrm{MeOH} / \mathrm{EtOAc}$. The solvent was evaporated and the residue subjected to flash chromatography ( $3 \% \mathrm{MeOH}$ in EtOAc) to afford $2.3 \mathrm{mg}(0.006 \mathrm{mmol}, 100 \%)$ of tetraol 59 as a white solid. mp $288-290{ }^{\circ} \mathrm{C} .{ }^{1} \mathrm{H}$ NMR $\left(400 \mathrm{MHz}, \mathrm{CDCl}_{3}\right.$ with $\left.10 \% \mathrm{CD}_{3} \mathrm{OD}\right) \delta$ 4.47 (s, 2H), 4.34 (s, 2H), 4.28 (q, 2H, $J=6.4 \mathrm{~Hz}), 4.23(\mathrm{~s}, 2 \mathrm{H}), 3.54(\mathrm{~m}, 4 \mathrm{H}), 2.98$ (s, $2 \mathrm{H}), 0.93(\mathrm{~d}, 6 \mathrm{H}, J=6.4 \mathrm{~Hz})$; NOED $\left(400 \mathrm{MHz}, \mathrm{CDCl}_{3}\right.$ with $\left.10 \% \mathrm{CD}_{3} \mathrm{OD}\right)$ : Irradiation at $\delta 3.54(\mathrm{H}-6): 4 \%$ enhancement at $\mathrm{H}_{-} 9,{ }^{13} \mathrm{C} \mathrm{NMR}\left(75.0 \mathrm{MHz}, \mathrm{CDCl}_{3}\right) \delta 133.9$, 
133.6, 79.6, 72.1, 65.2, 64.6, 55.3, 53.2, 29.2, 22.7; IR (thin film) (max 3406, 3020, 2927, $1653,1457,1376,1216,1049 \mathrm{~cm}^{-1}$; CIHRMS $[\mathrm{M}+\mathrm{H}]^{+}$calculated for $\mathrm{C}_{20} \mathrm{H}_{24} \mathrm{O}_{8}$ : 393.1551, found: $393.1522 ;[\alpha]_{\mathrm{D}}^{22}=-34^{\circ}\left(\mathrm{c}=0.2, \mathrm{CH}_{3} \mathrm{OH}\right)$.

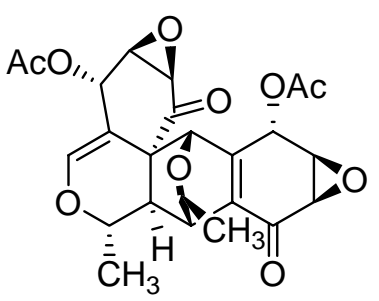

60

Bisacetyl-epoxyquinol A 60: To $6 \mathrm{mg}(0.015 \mathrm{mmol})$ of 6 and $0.1 \mathrm{mg}(0.0008 \mathrm{mmol})$ of DMAP in $0.8 \mathrm{~mL}$ of $\mathrm{CH}_{2} \mathrm{Cl}_{2}$ were added $4.5 \mu \mathrm{L}$ ( $0.047 \mathrm{mmol}, 3$ equiv.) of acetic anhydride and $10 \mu \mathrm{L}$ of triethylamine $(0.072 \mathrm{mmol}, 4.8$ equiv. $)$. After stirring at rt for $20 \mathrm{~min}, 4 \mathrm{~mL}$ of EtOAc was added and the organic layer was washed with brine, dried over $\mathrm{MgSO}_{4}$, filtered, and concentrated in vacuo to yield $7 \mathrm{mg}$ of $\mathbf{6 0}$ $(0.0148 \mathrm{mmol}, 95 \%)$ as pale yellow oil which was analytically pure for characterization. ${ }^{1} \mathrm{H}$ NMR $\left(400 \mathrm{MHz}, \mathrm{CDCl}_{3}\right) \delta 6.54(\mathrm{~s}, 1 \mathrm{H}), 5.96$ (s, 1H), $5.91(\mathrm{~s}, 1 \mathrm{H}), 5.01(\mathrm{~s}, 1 \mathrm{H}), 4.36(\mathrm{q}, 1 \mathrm{H}, J=6.8 \mathrm{~Hz}), 4.30(\mathrm{q}, 1 \mathrm{H}, J=6.4 \mathrm{~Hz}), 3.67$ $(\mathrm{d}, 1 \mathrm{H}, J=3.2 \mathrm{~Hz}), 3.64(\mathrm{~d}, 1 \mathrm{H}, J=3.2 \mathrm{~Hz}), 3.45(\mathrm{~d}, 1 \mathrm{H}, J=3.2 \mathrm{~Hz}), 3.37(\mathrm{~d}, 1 \mathrm{H}, J=3.2$ $\mathrm{Hz}), 3.14(\mathrm{~s}, 1 \mathrm{H}), 2.54(\mathrm{~s}, 1 \mathrm{H}), 2.17(\mathrm{~s}, 3 \mathrm{H}), 2.08(\mathrm{~s}, 3 \mathrm{H}), 1.03(\mathrm{~d}, 3 \mathrm{H}, J=6.8 \mathrm{~Hz}), 0.76$ $(\mathrm{d}, 3 \mathrm{H}, J=6.4 \mathrm{~Hz}) ;{ }^{13} \mathrm{C}$ NMR $\left(75.0 \mathrm{MHz}, \mathrm{CDCl}_{3}\right) \delta 198.7,188.4,169.6,169.5,147.6$, $142.0,136.8,108.4,74.3,71.1,66.8,64.9,64.0,63.4,55.8,54.8,52.4,49.5,38.6,36.8$, 20.9, 20.5, 20.3, 19.7; IR (thin film) vmax 2975, 2928, 1751, 1716, 1684, 1372, 1221, $1024 \mathrm{~cm}^{-1}$; CILRMS $\left[\mathrm{M}+\mathrm{NH}_{4}\right]^{+}$calculated for $\mathrm{C}_{24} \mathrm{H}_{28} \mathrm{O}_{10} \mathrm{~N}: 490.1$, found: $490.2 ;[\alpha]_{\mathrm{D}}{ }^{22}$ $=+96.7^{\circ}\left(\mathrm{c}=0.6, \mathrm{CHCl}_{3}\right)$.

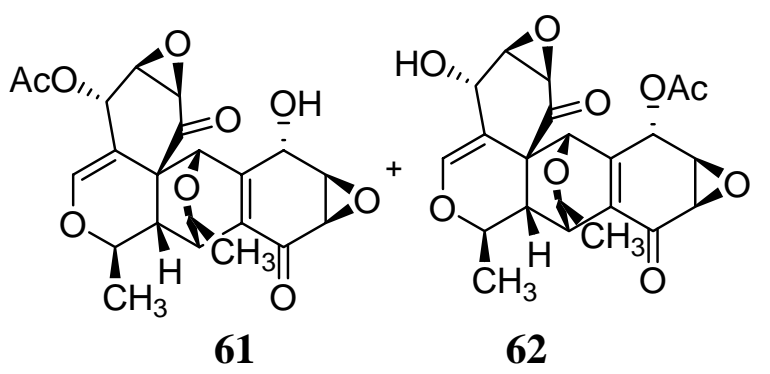

Monoacetyl-epoxyquinol B 61 and 62: To $10.5 \mathrm{mg}(0.027 \mathrm{mmol})$ of 7 and $0.6 \mathrm{mg}(0.005 \mathrm{mmol})$ of DMAP in 1 $\mathrm{mL}$ of $\mathrm{CH}_{2} \mathrm{Cl}_{2}$ were added $7.3 \mu \mathrm{L}$ (0.077 mmol, 2.9 equiv.) of acetic anhydride and $12.5 \mu \mathrm{L}$ of triethylamine $\left(0.090 \mathrm{mmol}, 3.5\right.$ equiv.) at $0^{\circ} \mathrm{C}$. After stirring at $0^{\circ} \mathrm{C}$ for $15 \mathrm{~min}$, volatiles were removed by an argon stream and the residue was subjected to flash chromatography ( $30 \%$ EtOAc in hexane) to afford $9.1 \mathrm{mg}(0.021 \mathrm{mmol}, 78 \%)$ of $\mathbf{6 1}$ as white solid and $2.5 \mathrm{mg}(0.0058 \mathrm{mmol}, 21 \%)$ of 62 as white solid.

61: mp 179-181 ${ }^{\circ} \mathrm{C} .{ }^{1} \mathrm{H}$ NMR (400 MHz, $\left.\mathrm{CDCl}_{3}\right) \delta 6.62(\mathrm{~s}, 1 \mathrm{H}), 5.67(\mathrm{~s}, 1 \mathrm{H}), 4.83(\mathrm{~s}$, $1 \mathrm{H}), 4.76(\mathrm{~d}, 1 \mathrm{H}, J=4.4 \mathrm{~Hz}), 4.18(\mathrm{q}, 1 \mathrm{H}, J=6.4 \mathrm{~Hz}), 3.82(\mathrm{dd}, 1 \mathrm{H}, J=1.6,3.6 \mathrm{~Hz})$, $3.66(\mathrm{dd}, 1 \mathrm{H}, J=2.0,2.4,3.2 \mathrm{~Hz}), 3.51(\mathrm{~m}, 3 \mathrm{H}), 3.11(\mathrm{~d}, 1 \mathrm{H}, J=2.8 \mathrm{~Hz}), 2.82(\mathrm{~d}, 1 \mathrm{H}, J$ $=3.6 \mathrm{~Hz}), 2.76(\mathrm{dd}, 1 \mathrm{H}, J=2.8,6.4 \mathrm{~Hz}), 2.24(\mathrm{~s}, 3 \mathrm{H}), 1.27(\mathrm{~d}, 3 \mathrm{H}, J=6.4 \mathrm{~Hz}), 0.78(\mathrm{~d}$, $3 \mathrm{H}, \mathrm{J}=6.4 \mathrm{~Hz}) ;{ }^{13} \mathrm{C}$ NMR $\left(75.0 \mathrm{MHz}, \mathrm{CDCl}_{3}\right) \delta 199.1,190.8,169.4,152.2,149.3$, 133.3, 102.7, 74.5, 73.1, 70.3, 70.0, 63.7, 56.0, 52.4, 51.1, 42.3, 36.7, 21.4, 19.9, 19.4; IR (thin film) vmax 3446, 2966, 2933, 1751, 1682, 1652, 1373, 1215, $1022 \mathrm{~cm}^{-1}$; CIHRMS 
$\mathrm{M}^{+}$calculated for $\mathrm{C}_{22} \mathrm{H}_{22} \mathrm{O}_{9}: 430.1264$, found: $430.1303 ;[\alpha]_{\mathrm{D}}^{22}=+162^{\circ}$ (c $=1.0$, $\left.\mathrm{CHCl}_{3}\right)$.

62: mp 104-107 ${ }^{\circ} \mathrm{C} .{ }^{1} \mathrm{H}$ NMR $\left(400 \mathrm{MHz}, \mathrm{CDCl}_{3}\right) \delta 6.47(\mathrm{~s}, 1 \mathrm{H}), 5.94(\mathrm{~s}, 1 \mathrm{H}), 5.08(\mathrm{~s}$, $1 \mathrm{H}), 4.49(\mathrm{~s}, 1 \mathrm{H}), 4.14(\mathrm{q}, 1 \mathrm{H}, J=6.4 \mathrm{~Hz}), 3.74(\mathrm{dd}, 1 \mathrm{H}, J=1.6,3.6 \mathrm{~Hz}), 3.54(\mathrm{~d}, 1 \mathrm{H}, J$ $=1.2 \mathrm{~Hz}), 3.51(\mathrm{~d}, 1 \mathrm{H}, J=3.6 \mathrm{~Hz}), 3.47(\mathrm{~d}, 1 \mathrm{H}, J=1.6 \mathrm{~Hz}), 3.37(\operatorname{app} \mathrm{p}, 1 \mathrm{H}, J=6.4 \mathrm{~Hz})$, $3.10(\mathrm{dd}, 1 \mathrm{H}, J=1.2,3.2 \mathrm{~Hz}), 2.77(\mathrm{dd}, 1 \mathrm{H}, J=3.2,8.0 \mathrm{~Hz}), 2.10(\mathrm{~s}, 3 \mathrm{H}), 1.30(\mathrm{~d}, 3 \mathrm{H}, J$ $=6.4 \mathrm{~Hz}), 0.77(\mathrm{~d}, 3 \mathrm{H}, J=6.4 \mathrm{~Hz}) ;{ }^{13} \mathrm{C} \mathrm{NMR}\left(75.0 \mathrm{MHz}, \mathrm{CDCl}_{3}\right) \delta 199.0,190.8,169.8$, $149.5,145.1,136.5,105.6,94.5,74.2,72.9,69.4,68.2,65.6,54.7,53.9,52.4,52.2,41.5$, 37.0, 21.1, 20.1, 19.2; IR (thin film) vmax 3446, 2971, 2926, 1684, 1229, $1027 \mathrm{~cm}^{-1}$; CIHRMS $[\mathrm{M}+\mathrm{H}]^{+}$calculated for $\mathrm{C}_{22} \mathrm{H}_{23} \mathrm{O}_{9}: 431.1344$, found: $431.1288 ;[\alpha]_{\mathrm{D}}{ }^{22}=+66.7^{\circ}$ $\left(\mathrm{c}=0.3, \mathrm{CHCl}_{3}\right)$.

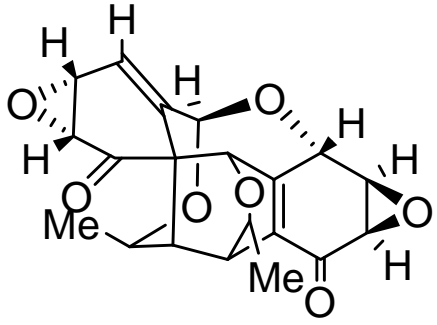

63

Ether 63: Epoxyquinol B $7(16 \mathrm{mg}, 0.037 \mathrm{mmol})$ in 2.5 $\mathrm{mL}$ of THF was irradiated at $150{ }^{\circ} \mathrm{C}$ for $15 \mathrm{~min}$ using the CEM Discover ${ }^{\mathrm{TM}}$ explorer microwave system. The reaction mixture was concentrated in vacuo and directly subjected to silica gel chromatography (10\% EtOAc in $\left.\mathrm{CH}_{2} \mathrm{Cl}_{2}\right)$ to afford $8 \mathrm{mg}(0.022 \mathrm{mmol}, 58 \%)$ of 63 as white solid. mp $125-127{ }^{\circ} \mathrm{C} .{ }^{1} \mathrm{H}$ NMR $\left(400 \mathrm{MHz}, \mathrm{CDCl}_{3}\right)$ $\delta 6.41(\mathrm{~d}, 1 \mathrm{H}, J=3.2 \mathrm{~Hz}), 5.37(\mathrm{~s}, 1 \mathrm{H}), 4.84(\mathrm{~d}, 1 \mathrm{H}, J=$ $2.4 \mathrm{~Hz}), 4.45(\mathrm{q}, 1 \mathrm{H}, J=6.4 \mathrm{~Hz}), 4.29(\mathrm{~s}, 1 \mathrm{H}), 3.92(\mathrm{dd}$, $1 \mathrm{H}, J=2.0,4.0 \mathrm{~Hz}), 3.83(\mathrm{q}, 1 \mathrm{H}, J=7.2 \mathrm{~Hz}), 3.77(\mathrm{~m}, 2 \mathrm{H}), 3.63(\mathrm{~d}, 1 \mathrm{H}, J=4.0 \mathrm{~Hz})$, $3.43(\mathrm{~d}, 1 \mathrm{H}, J=2.0 \mathrm{~Hz}), 2.57(\mathrm{~d}, 1 \mathrm{H}, J=2.8 \mathrm{~Hz}), 0.93(\mathrm{~d}, 3 \mathrm{H}, J=6.4 \mathrm{~Hz}), 0.90$ (d, 3H, $J$ $=7.2 \mathrm{~Hz}) ;{ }^{13} \mathrm{C} \mathrm{NMR}\left(75.0 \mathrm{MHz}, \mathrm{CDCl}_{3}\right) \delta 198.0,190.5,147.0,140.5,138.7,125.1$, $97.9,72.5,70.1,67.1,60.4,54.5,53.0,50.1,49.6,43.9,40.2,21.8,20.3$; IR (thin film) vmax 3022, 2973, 2929, 1717, 1683, 1380, 1226, 1055, $1015 \mathrm{~cm}^{-1}$; CIHRMS [M+H] calculated for $\mathrm{C}_{20} \mathrm{H}_{19} \mathrm{O}_{7}: 371.1133$, found: 371.1097 ; $[\alpha]_{\mathrm{D}}{ }^{22}=+332^{\circ}\left(\mathrm{c}=0.5, \mathrm{CHCl}_{3}\right)$.

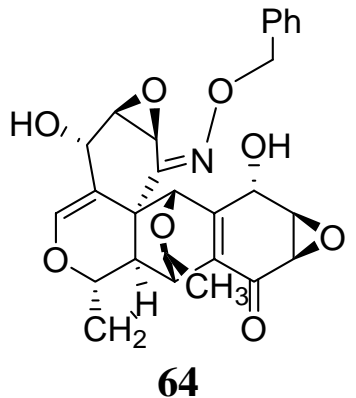

Oxime 64: Epoxyquinol A 6 (9 $\mathrm{mg}, 0.023 \mathrm{mmol})$ and $7 \mu \mathrm{L}$ $(0.083 \mathrm{mmol})$ of $O$-benzyl hydroxylamine in $0.5 \mathrm{~mL}$ EtOAc was added $4 \mu \mathrm{L}(0.07 \mathrm{mmol})$ of acetic acid. After stirring at $\mathrm{rt}$ for $1.5 \mathrm{~h}$, volatiles were removed by an argon stream and the residue subjected to flash chromatography (50\% EtOAc in hexane) to afford $11.3 \mathrm{mg}(0.023 \mathrm{mmol}, 100 \%)$ of $\mathbf{6 4}$ as white solid. mp 154-156 ${ }^{\circ} \mathrm{C} .{ }^{1} \mathrm{H}$ NMR (400 MHz, $\left.\mathrm{CD}_{3} \mathrm{OD}\right) \delta 7.36$ $(\mathrm{m}, 5 \mathrm{H}), 6.68(\mathrm{~d}, 1 \mathrm{H}, J=2.0 \mathrm{~Hz}), 5.24(\mathrm{~s}, 2 \mathrm{H}), 5.13(\mathrm{~s}, 1 \mathrm{H})$, $4.46(\mathrm{~s}, 1 \mathrm{H}), 4.28(\mathrm{~m}, 3 \mathrm{H}), 3.69(\mathrm{~d}, 1 \mathrm{H}, J=3.6 \mathrm{~Hz}), 3.42(\mathrm{~m}$, $2 \mathrm{H}), 3.18(\mathrm{~s}, 1 \mathrm{H}), 2.60(\mathrm{~s}, 1 \mathrm{H}), 1.03(\mathrm{~d}, 3 \mathrm{H}, J=6.4 \mathrm{~Hz}), 0.71(\mathrm{~d}, 3 \mathrm{H}, J=6.4 \mathrm{~Hz}) ;{ }^{13} \mathrm{C}$ NMR $\left(75.0 \mathrm{MHz}, \mathrm{CD}_{3} \mathrm{OD}\right) \delta 202.4,150.2,147.1,143.2,140.0,139.0,132.3,130.0$, $129.7,129.3,116.0,78.2,75.9,73.1,68.0,67.2$, 64.6, 64.3, 57.4, 57.0, 52.1, 45.1, 41.2, 38.4, 21.0, 20.4; IR (thin film) vmax 3420, 2973, 2927, 2872, 1707, 1653, 1375, 1253, 1202, 1037, $1012 \mathrm{~cm}^{-1}$; CIHRMS $\mathrm{M}^{+}$calculated for $\mathrm{C}_{27} \mathrm{H}_{26} \mathrm{NO}_{8}$ : 492.1658, found: $492.1706 ;[\alpha]_{\mathrm{D}}^{22}=+10.5^{\circ}(\mathrm{c}=1.0$, EtOAc $)$. 


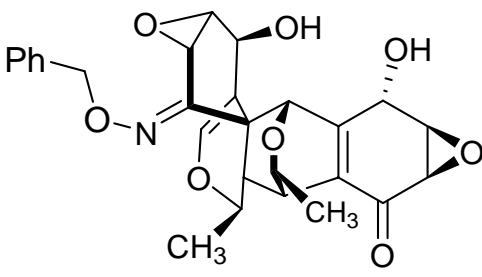

65

Oxime 65: Epoxyquinol B 7 (7.5 $\mathrm{mg}, 0.019 \mathrm{mmol})$ and $5 \mu \mathrm{L}(0.059 \mathrm{mmol})$ of $O$-benzylhydroxylamine in $0.5 \mathrm{~mL}$ EtOAc was added $5 \mu \mathrm{L}(0.088 \mathrm{mmol})$ of acetic acid. The reaction mixture was irradiated at $110{ }^{\circ} \mathrm{C}$ for 50 min using the CEM Discover ${ }^{\mathrm{TM}}$ explorer microwave system and then concentrated in vacuo and subjected to silica gel chromatography $(30 \%$ EtOAc in hexane) to afford $6.5 \mathrm{mg}(0.013 \mathrm{mmol}, 68 \%)$ of 65 as a pale yellow oil and $2 \mathrm{mg}$ (93\% based on recovered starting material) of $7 .{ }^{1} \mathrm{H} \mathrm{NMR}$ $\left(400 \mathrm{MHz}, \mathrm{CDCl}_{3}\right) \delta 7.33(\mathrm{~m}, 5 \mathrm{H}), 6.50(\mathrm{~s}, 1 \mathrm{H}), 5.24(\mathrm{~d}, 1 \mathrm{H}, J=12.4 \mathrm{~Hz}), 5.16(\mathrm{~d}, 1 \mathrm{H}, J$ $=12.4 \mathrm{~Hz}), 5.00(\mathrm{~s}, 1 \mathrm{H}), 4.72(\mathrm{~s}, 1 \mathrm{H}), 4.53(\mathrm{~s}, 1 \mathrm{H}), 4.35(\mathrm{~d}, 1 \mathrm{H}, J=4.0 \mathrm{~Hz}), 4.09(\mathrm{q}, 1 \mathrm{H}$, $J=6.0 \mathrm{~Hz}), 3.59(\mathrm{~m}, 2 \mathrm{H}), 3.46(\mathrm{~d}, 1 \mathrm{H}, J=3.2 \mathrm{~Hz}), 3.41(\operatorname{app~p}, 1 \mathrm{H}, J=7.2 \mathrm{~Hz}), 3.13(\mathrm{~d}$, $1 \mathrm{H}, J=2.0 \mathrm{~Hz}), 2.76(\mathrm{dd}, 1 \mathrm{H}, J=3.2,8.0 \mathrm{~Hz}), 1.17(\mathrm{~d}, 3 \mathrm{H}, J=6.0 \mathrm{~Hz}), 0.75(\mathrm{~d}, 3 \mathrm{H}, J=$ $6.0 \mathrm{~Hz}) ;{ }^{13} \mathrm{C} \mathrm{NMR}\left(75.0 \mathrm{MHz}, \mathrm{CDCl}_{3}\right) \delta 199.5,150.3,149.5,137.0,136.3,130.5$, $128.5,128.4,128.3,105.9,74.5,72.9,69.6,68.2,64.0,60.5,55.1,54.5,52.8,52.3,43.4$, 40.9, 37.2, 20.1, 19.3; IR (thin film) $(\max 3373,2972,2926,2856,1727,1653,1456$, 1381, 1192, $1016 \mathrm{~cm}^{-1}$; CIHRMS [M+H] ${ }^{+}$calculated for $\mathrm{C}_{27} \mathrm{H}_{27} \mathrm{NO}_{8}$ : 493.1738, found: 493.1747; $[\alpha]_{\mathrm{D}}^{22}=+37^{\circ}\left(\mathrm{c}=1.0, \mathrm{CHCl}_{3}\right)$.

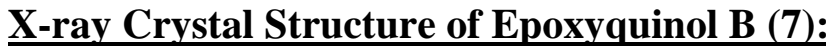

Two molecules in the asymmetric unit
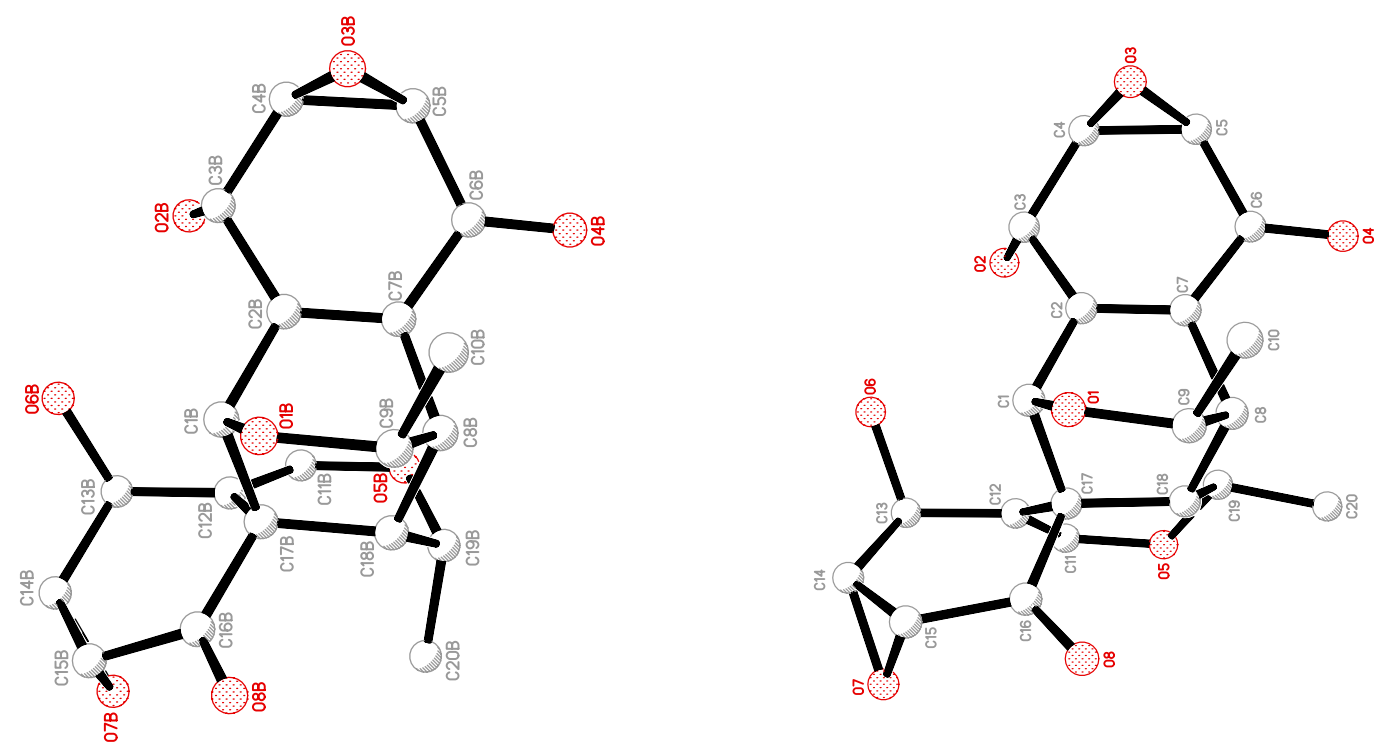
Crystals of 7 suitable for x-ray analysis were obtained by slow evaporation from Hexane/Ether/THF. Crystallographic data have been deposited with the Cambridge Crystallographic Data Centre (CCDC \#188062). Copies of the data can be obtained free of charge on application to the CCDC, 12 Union Road, Cambridge CB21EZ, UK (fax: (+44)-1223-336-033; e-mail: deposit@ccdc.cam.ac.uk.

Table 1. Crystal data and structure refinement for 7

Identification code

Empirical formula

Formula weight

Temperature

Wavelength

Crystal system

Space group

Unit cell dimensions

Volume

Z

Density (calculated)

Absorption coefficient

$\mathrm{F}(000)$

Crystal size

Theta range for data collection

Index ranges

Reflections collected

Independent reflections

Completeness to theta $=20.81^{\circ}$

Absorption correction

Max. and min. transmission

Refinement method

Data / restraints / parameters

Goodness-of-fit on $\mathrm{F}^{2}$

Final R indices [I $>2 \operatorname{sigma}(\mathrm{I})]$

$\mathrm{R}$ indices (all data)

Absolute structure parameter

Largest diff. peak and hole

\section{7}

C23.33 H28.33 O8.83

450.13

173(2) K

$0.71073 \AA$

Orthorhombic

P2(1)2(1)2(1)

$\mathrm{a}=10.1647(3) \AA \quad \alpha=90^{\circ}$.

$\mathrm{b}=10.1944(3) \AA \quad \beta=90^{\circ}$.

$\mathrm{c}=67.375(2) \AA \quad \gamma=90^{\circ}$.

6981.6(4) $\AA^{3}$

12

$1.285 \mathrm{Mg} / \mathrm{m}^{3}$

$0.099 \mathrm{~mm}^{-1}$

2868

$0.50 \times 0.30 \times 0.20 \mathrm{~mm}^{3}$

2.83 to $20.81^{\circ}$.

$-10<=\mathrm{h}<=9,-10<=\mathrm{k}<=10,-59<=1<=56$

25857

$5959[\mathrm{R}(\mathrm{int})=0.0583]$

$86.9 \%$

SADABS

0.9806 and 0.8524

Full-matrix least-squares on $\mathrm{F}^{2}$

$5959 / 0 / 824$

1.107

$\mathrm{R} 1=0.0743, \mathrm{wR} 2=0.1798$

$\mathrm{R} 1=0.0874, \mathrm{wR} 2=0.1874$

$0(2)$

0.932 and -0.271 e. $\AA^{-3}$

Table 2. Atomic coordinates ( $\left.\times 10^{4}\right)$ and equivalent isotropic displacement parameters $\left(\AA^{2} \times 10^{3}\right)$ for 7. $U(e q)$ is defined as one third of the trace of the orthogonalized $U^{i j}$ tensor.

\begin{tabular}{lrrrr}
\hline & $\mathrm{x}$ & $\mathrm{y}$ & $\mathrm{z}$ & $\mathrm{U}(\mathrm{eq})$ \\
\hline $\mathrm{O}(1)$ & & & & \\
$\mathrm{O}(2)$ & $1142(4)$ & $11093(4)$ & $1036(1)$ & $24(1)$ \\
$\mathrm{O}(3)$ & $4231(4)$ & $7924(4)$ & $897(1)$ & $34(1)$ \\
$\mathrm{O}(4)$ & $5528(4)$ & $11062(5)$ & $1069(1)$ & $43(2)$ \\
$\mathrm{O}(5)$ & $4476(4)$ & $9925(6)$ & $1529(1)$ & $52(2)$ \\
$\mathrm{O}(6)$ & $1055(5)$ & $6446(4)$ & $1318(1)$ & $45(2)$ \\
$\mathrm{O}(7)$ & $1916(4)$ & $7631(4)$ & $679(1)$ & $31(1)$ \\
$\mathrm{O}(8)$ & $-1643(4)$ & $7588(4)$ & $791(1)$ & $42(2)$ \\
$\mathrm{C}(1)$ & $-1417(4)$ & $9732(5)$ & $1132(1)$ & $39(1)$ \\
$\mathrm{C}(2)$ & $1640(5)$ & $9814(5)$ & $974(1)$ & $18(2)$ \\
$\mathrm{C}(3)$ & $3012(5)$ & $9679(5)$ & $1049(1)$ & $19(2)$ \\
$\mathrm{C}(4)$ & $4151(6)$ & $9329(6)$ & $921(1)$ & $26(2)$ \\
& $5462(6)$ & $9730(7)$ & $1007(1)$ & $29(2)$
\end{tabular}




\begin{tabular}{|c|c|c|c|c|}
\hline$C(5)$ & $5584(6)$ & $10043(7)$ & $1220(1)$ & $37(2)$ \\
\hline$C(6)$ & $4378(6)$ & $9961(6)$ & $1347(1)$ & $32(2)$ \\
\hline$C(7)$ & $3100(6)$ & $9937(6)$ & 1241(1) & $24(2)$ \\
\hline$C(8)$ & $1780(6)$ & $10092(6)$ & 1345(1) & $27(2)$ \\
\hline C(9) & 1107(6) & $11285(7)$ & 1244(1) & $29(2)$ \\
\hline$C(10)$ & $1727(8)$ & $12578(7)$ & $1295(1)$ & $52(3)$ \\
\hline$C(11)$ & $961(6)$ & $6363(7)$ & 1113(1) & $38(2)$ \\
\hline$C(12)$ & $901(6)$ & 7397(6) & $996(1)$ & $27(2)$ \\
\hline $\mathrm{C}(13)$ & $757(6)$ & $7140(6)$ & $780(1)$ & $29(2)$ \\
\hline$C(14)$ & $-416(6)$ & $7880(7)$ & $692(1)$ & $33(2)$ \\
\hline$C(15)$ & $-1099(6)$ & $8890(7)$ & 814(1) & $32(2)$ \\
\hline$C(16)$ & $-668(6)$ & 9168(6) & 1024(1) & $26(2)$ \\
\hline$C(17)$ & $726(5)$ & 8772(6) & 1079(1) & $18(2)$ \\
\hline$C(18)$ & $964(6)$ & $8862(6)$ & $1302(1)$ & $30(2)$ \\
\hline$C(19)$ & 1649(7) & 7641(7) & 1391(1) & $46(2)$ \\
\hline$C(20)$ & $1595(10)$ & 7601(8) & 1619(1) & $70(3)$ \\
\hline $\mathrm{O}(1 \mathrm{~A})$ & $5022(4)$ & $875(4)$ & $2287(1)$ & $26(1)$ \\
\hline $\mathrm{O}(2 \mathrm{~A})$ & 8141(4) & $-2200(5)$ & $2459(1)$ & $40(2)$ \\
\hline $\mathrm{O}(3 \mathrm{~A})$ & 5071(4) & $-3458(4)$ & 2252(1) & $36(1)$ \\
\hline $\mathrm{O}(4 \mathrm{~A})$ & 6552(6) & $-2487(5)$ & 1810(1) & $63(2)$ \\
\hline $\mathrm{O}(5 \mathrm{~A})$ & 9753(4) & $1052(5)$ & 2024(1) & $40(2)$ \\
\hline $\mathrm{O}(6 \mathrm{~A})$ & 8403(4) & $130(4)$ & $2652(1)$ & $36(2)$ \\
\hline $\mathrm{O}(7 \mathrm{~A})$ & $8496(5)$ & 3692(4) & $2547(1)$ & $47(2)$ \\
\hline $\mathrm{O}(8 \mathrm{~A})$ & 6411(4) & 3450(4) & 2195(1) & $40(2)$ \\
\hline$C(1 \mathrm{~A})$ & $6300(5)$ & $419(5)$ & $2358(1)$ & $21(2)$ \\
\hline$C(2 A)$ & $6462(6)$ & $-991(6)$ & 2281(1) & $25(2)$ \\
\hline $\mathrm{C}(3 \mathrm{~A})$ & $6759(6)$ & $-2109(6)$ & 2422(1) & $18(2)$ \\
\hline$C(4 A)$ & 6381(7) & $-3391(6)$ & $2328(1)$ & $31(2)$ \\
\hline$C(5 \mathrm{~A})$ & $6163(7)$ & $-3518(7)$ & $2119(1)$ & $40(2)$ \\
\hline$C(6 \mathrm{~A})$ & $6365(7)$ & $-2348(7)$ & 1986(1) & $35(2)$ \\
\hline$C(7 A)$ & $6298(6)$ & $-1090(6)$ & $2082(1)$ & $23(2)$ \\
\hline $\mathrm{C}(8 \mathrm{~A})$ & $6097(5)$ & 191(6) & 1979(1) & $20(2)$ \\
\hline$C(9 \mathrm{~A})$ & $4885(7)$ & $840(7)$ & $2070(1)$ & $31(2)$ \\
\hline$C(10 A)$ & $3604(6)$ & $178(8)$ & $2021(1)$ & $41(2)$ \\
\hline$C(11 \mathrm{~A})$ & $9770(7)$ & $1129(7)$ & $2220(1)$ & $41(2)$ \\
\hline$C(12 A)$ & $8738(7)$ & 1159(6) & $2340(1)$ & $31(2)$ \\
\hline$C(13 A)$ & $8905(7)$ & 1304(6) & 2561(1) & $37(2)$ \\
\hline$C(14 A)$ & $8179(6)$ & 2449(6) & $2643(1)$ & $37(2)$ \\
\hline$C(15 A)$ & $7163(6)$ & 3132(7) & 2523(1) & $31(2)$ \\
\hline$C(16 A)$ & $6940(6)$ & $2704(6)$ & $2314(1)$ & $31(2)$ \\
\hline$C(17 A)$ & $7383(6)$ & 1312(6) & $2253(1)$ & $20(2)$ \\
\hline$C(18 \mathrm{~A})$ & $7313(6)$ & 1051(6) & 2028(1) & $23(2)$ \\
\hline$C(19 A)$ & $8569(7)$ & $405(7)$ & 1950(1) & $33(2)$ \\
\hline$C(20 A)$ & $8681(7)$ & $485(9)$ & 1727(1) & $57(3)$ \\
\hline $\mathrm{O}(1 \mathrm{~B})$ & $-3655(4)$ & $10886(4)$ & $596(1)$ & $27(1)$ \\
\hline $\mathrm{O}(2 \mathrm{~B})$ & $-687(4)$ & 14091(4) & 771(1) & $36(1)$ \\
\hline $\mathrm{O}(3 \mathrm{~B})$ & 493(4) & $11114(4)$ & $528(1)$ & $35(1)$ \\
\hline $\mathrm{O}(4 \mathrm{~B})$ & $-597(4)$ & $12577(5)$ & $99(1)$ & $44(2)$ \\
\hline $\mathrm{O}(5 \mathrm{~B})$ & $-3279(4)$ & $15201(4)$ & $304(1)$ & $36(1)$ \\
\hline $\mathrm{O}(6 \mathrm{~B})$ & $-3094(4)$ & $14346(4)$ & $966(1)$ & $35(1)$ \\
\hline $\mathrm{O}(7 \mathrm{~B})$ & $-6506(5)$ & 14893(5) & 796(1) & $50(2)$ \\
\hline $\mathrm{O}(8 \mathrm{~B})$ & $-6368(4)$ & 11982(4) & $587(1)$ & $36(1)$ \\
\hline$C(1 B)$ & $-3363(6)$ & $12213(6)$ & $660(1)$ & $26(2)$ \\
\hline $\mathrm{C}(2 \mathrm{~B})$ & $-2005(5)$ & $12502(6)$ & $578(1)$ & $21(2)$ \\
\hline$C(3 B)$ & $-827(6)$ & $12750(6)$ & $715(1)$ & $25(2)$ \\
\hline $\mathrm{C}(4 \mathrm{~B})$ & $438(6)$ & $12398(6)$ & $621(1)$ & $29(2)$ \\
\hline
\end{tabular}




$\begin{array}{lcccc}\mathrm{C}(5 \mathrm{~B}) & 525(6) & 12264(7) & 402(1) & 37(2) \\ \mathrm{C}(6 \mathrm{~B}) & -670(7) & 12444(6) & 280(1) & 30(2) \\ \mathrm{C}(7 \mathrm{~B}) & -1937(5) & 12396(6) & 381(1) & 18(2) \\ \mathrm{C}(8 \mathrm{~B}) & -3218(6) & 12018(6) & 292(1) & 21(2) \\ \mathrm{C}(9 \mathrm{~B}) & -3620(6) & 10718(6) & 382(1) & 22(2) \\ \mathrm{C}(10 \mathrm{~B}) & -2742(7) & 9565(6) & 331(1) & 41(2) \\ \mathrm{C}(11 \mathrm{~B}) & -3402(6) & 15397(6) & 501(1) & 26(2) \\ \mathrm{C}(12 \mathrm{~B}) & -3943(6) & 14574(6) & 629(1) & 24(2) \\ \mathrm{C}(13 \mathrm{~B}) & -4070(6) & 14983(7) & 840(1) & 37(2) \\ \mathrm{C}(14 \mathrm{~B}) & -5421(6) & 14619(8) & 924(1) & 44(2) \\ \mathrm{C}(15 \mathrm{~B}) & -6181(6) & 13542(7) & 836(1) & 34(2) \\ \mathrm{C}(16 \mathrm{~B}) & -5683(6) & 12856(6) & 654(1) & 24(2) \\ \mathrm{C}(17 \mathrm{~B}) & -4356(6) & 13203(6) & 569(1) & 20(2) \\ \mathrm{C}(18 \mathrm{~B}) & -4303(6) & 13025(6) & 341(1) & 24(2) \\ \mathrm{C}(19 \mathrm{~B}) & -4267(6) & 14287(6) & 227(1) & 31(2) \\ \mathrm{C}(20 \mathrm{~B}) & -5600(7) & 14973(7) & 226(1) & 45(2) \\ \mathrm{C}(1 \mathrm{~S}) & 253(9) & 5576(9) & 251(2) & 66(3) \\ \mathrm{C}(2 \mathrm{~S}) & 414(8) & 6891(8) & 167(1) & 56(2) \\ \mathrm{O}(3 \mathrm{~S}) & 1535(4) & 7492(4) & 266(1) & 38(1) \\ \mathrm{C}(4 \mathrm{~S}) & 1795(8) & 8740(8) & 179(1) & 59(2) \\ \mathrm{C}(5 \mathrm{~S}) & 3000(7) & 9326(8) & 270(1) & 52(2) \\ \mathrm{C}(6 \mathrm{~S}) & -870(10) & 5087(11) & 1963(2) & 97(4) \\ \mathrm{C}(7 \mathrm{~S}) & 160(9) & 4431(10) & 1855(2) & 72(3) \\ \mathrm{O}(8 \mathrm{~S}) & 1361(5) & 4796(5) & 1939(1) & 48(1) \\ \mathrm{C}(9 \mathrm{~S}) & 2409(9) & 4083(11) & 1848(2) & 86(3) \\ \mathrm{C}(10 \mathrm{~S}) & 3592(10) & 4528(11) & 1938(2) & 93(3) \\ \mathrm{C}(11 \mathrm{~S}) & -2761(16) & 16960(16) & 1334(3) & 58(5) \\ \mathrm{C}(12 \mathrm{~S}) & -2510(40) & 16080(40) & 1413(5) & 185(14) \\ \mathrm{O}(13 \mathrm{~S}) & -3596(9) & 14987(9) & 1343(2) & 42(3) \\ \mathrm{C}(14 \mathrm{~S}) & -3580(40) & 14080(40) & 1477(6) & 199(15) \\ \mathrm{C}(15 \mathrm{~S}) & -4220(15) & 13050(15) & 1491(3) & 49(4) \\ & & & & \end{array}$

\section{$\underline{\text { X-ray Crystal Structure of RKB-3564D (8) }}$}

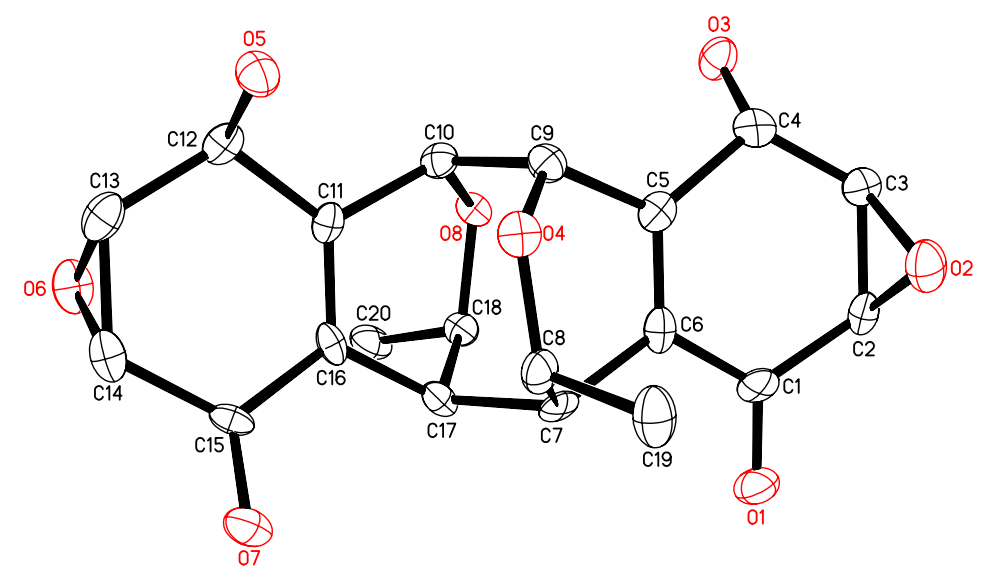


Crystals of RKB-3564D (8) suitable for x-ray analysis were obtained by slow evaporation from EtOAc/Ether. Crystallographic data have been deposited with the Cambridge Crystallographic Data Centre (CCDC \#220119). Copies of the data can be obtained free of charge on application to the CCDC, 12 Union Road, Cambridge CB21EZ, UK (fax: (+44)-1223-336-033; e-mail: deposit@ccdc.cam.ac.uk.

\begin{tabular}{|c|c|c|}
\hline Identification code & \multicolumn{2}{|l|}{ RKB-3564D (8) } \\
\hline Empirical formula & \multicolumn{2}{|l|}{$\mathrm{C} 20 \mathrm{H} 20 \mathrm{O} 8$} \\
\hline Formula weight & \multicolumn{2}{|l|}{388.36} \\
\hline Temperature & \multicolumn{2}{|l|}{$125(2) \mathrm{K}$} \\
\hline Wavelength & \multicolumn{2}{|l|}{$0.71073 \AA$} \\
\hline Crystal system & \multicolumn{2}{|l|}{ Monoclinic } \\
\hline Space group & \multicolumn{2}{|l|}{$\mathrm{P} 2(1)$} \\
\hline \multirow[t]{3}{*}{ Unit cell dimensions } & $\mathrm{a}=8.256(6) \AA$ & $\alpha=90^{\circ}$. \\
\hline & $\mathrm{b}=7.118(5) \AA$ & $\beta=96.259(19)^{\circ}$ \\
\hline & $\mathrm{c}=14.087(11) \AA$ & $\gamma=90^{\circ}$ \\
\hline Volume & \multicolumn{2}{|l|}{$823.0(10) \AA^{3}$} \\
\hline Z & \multicolumn{2}{|l|}{2} \\
\hline Density (calculated) & \multicolumn{2}{|l|}{$1.567 \mathrm{Mg} / \mathrm{m}^{3}$} \\
\hline Absorption coefficient & \multicolumn{2}{|l|}{$0.122 \mathrm{~mm}^{-1}$} \\
\hline $\mathrm{F}(000)$ & \multicolumn{2}{|l|}{408} \\
\hline Crystal size & \multicolumn{2}{|c|}{$0.20 \times 0.04 \times 0.04 \mathrm{~mm}^{3}$} \\
\hline Theta range for data collection & \multicolumn{2}{|l|}{2.48 to $25.42^{\circ}$. } \\
\hline Index ranges & \multicolumn{2}{|c|}{$-9<=\mathrm{h}<=8,-6<=\mathrm{k}<=8,-11<=1<=16$} \\
\hline Reflections collected & \multicolumn{2}{|c|}{3254} \\
\hline Independent reflections & \multicolumn{2}{|c|}{$2285[\mathrm{R}($ int $)=0.0452]$} \\
\hline Completeness to theta $=25.42^{\circ}$ & \multicolumn{2}{|l|}{$94.6 \%$} \\
\hline Absorption correction & \multicolumn{2}{|l|}{ SADABS } \\
\hline Max. and min. transmission & \multicolumn{2}{|l|}{0.9951 and 0.9760} \\
\hline Refinement method & \multicolumn{2}{|c|}{ Full-matrix least-squares on $\mathrm{F}^{2}$} \\
\hline Data / restraints / parameters & \multicolumn{2}{|l|}{$2285 / 1 / 297$} \\
\hline Goodness-of-fit on $\mathrm{F}^{2}$ & \multicolumn{2}{|l|}{1.011} \\
\hline Final $\mathrm{R}$ indices $[\mathrm{I}>2 \operatorname{sigma}(\mathrm{I})]$ & \multicolumn{2}{|c|}{$\mathrm{R} 1=0.0524, \mathrm{wR} 2=0.1051$} \\
\hline $\mathrm{R}$ indices (all data) & \multicolumn{2}{|c|}{$\mathrm{R} 1=0.0816, \mathrm{wR} 2=0.1169$} \\
\hline Absolute structure parameter & \multicolumn{2}{|l|}{$-0.5(19)$} \\
\hline Largest diff. peak and hole & \multicolumn{2}{|c|}{0.255 and -0.265 e. $\AA^{-3}$} \\
\hline
\end{tabular}

Table 2. Atomic coordinates $\left(\mathrm{x} 10^{4}\right)$ and equivalent isotropic displacement parameters $\left(\AA^{2} \times 10^{3}\right)$ for 8. $U(e q)$ is defined as one third of the trace of the orthogonalized $U^{i j}$ tensor.

\begin{tabular}{|c|c|c|c|c|}
\hline & $\mathrm{x}$ & $\mathrm{y}$ & z & $\mathrm{U}(\mathrm{eq})$ \\
\hline $\mathrm{O}(1)$ & $-5532(3)$ & $6213(3)$ & $7746(2)$ & $26(1)$ \\
\hline $\mathrm{O}(2)$ & $-6449(3)$ & $3692(4)$ & $9622(2)$ & $26(1)$ \\
\hline $\mathrm{O}(3)$ & $-7082(3)$ & $-495(4)$ & $8159(2)$ & $22(1)$ \\
\hline $\mathrm{O}(4)$ & $-1707(3)$ & $930(3)$ & $8582(2)$ & $16(1)$ \\
\hline $\mathrm{O}(5)$ & $-66(3)$ & $-2565(4)$ & $7732(2)$ & $20(1)$ \\
\hline $\mathrm{O}(6)$ & $638(3)$ & $-755(4)$ & $5366(2)$ & $23(1)$ \\
\hline $\mathrm{O}(7)$ & 1012(3) & $3530(3)$ & $6585(2)$ & $22(1)$ \\
\hline $\mathrm{O}(8)$ & $-4541(3)$ & $380(3)$ & $6515(2)$ & $13(1)$ \\
\hline $\mathrm{C}(1)$ & $-5624(4)$ & $4661(5)$ & $8097(3)$ & $18(1)$ \\
\hline $\mathrm{C}(2)$ & $-7015(4)$ & $4208(5)$ & $8646(3)$ & $17(1)$ \\
\hline$C(3)$ & $-7181(4)$ & $2281(5)$ & 8994(3) & $16(1)$ \\
\hline$C(4)$ & $-6074(4)$ & $760(5)$ & $8752(3)$ & $15(1)$ \\
\hline
\end{tabular}




\begin{tabular}{lrrrr}
$\mathrm{C}(5)$ & $-4623(4)$ & $1427(5)$ & $8299(3)$ & $15(1)$ \\
$\mathrm{C}(6)$ & $-4407(4)$ & $3195(5)$ & $8009(3)$ & $14(1)$ \\
$\mathrm{C}(7)$ & $-2787(4)$ & $3655(5)$ & $7665(3)$ & $17(1)$ \\
$\mathrm{C}(8)$ & $-1476(4)$ & $2904(5)$ & $8410(3)$ & $18(1)$ \\
$\mathrm{C}(9)$ & $-3244(4)$ & $118(5)$ & $8195(3)$ & $17(1)$ \\
$\mathrm{C}(10)$ & $-3276(4)$ & $-536(5)$ & $7131(3)$ & $15(1)$ \\
$\mathrm{C}(11)$ & $-1629(4)$ & $-249(5)$ & $6789(3)$ & $15(1)$ \\
$\mathrm{C}(12)$ & $-505(4)$ & $-1905(5)$ & $6791(3)$ & $15(1)$ \\
$\mathrm{C}(13)$ & $963(4)$ & $-1518(6)$ & $6306(3)$ & $22(1)$ \\
$\mathrm{C}(14)$ & $1423(4)$ & $424(6)$ & $6110(3)$ & $20(1)$ \\
$\mathrm{C}(15)$ & $432(4)$ & $1999(5)$ & $6421(2)$ & $15(1)$ \\
$\mathrm{C}(16)$ & $-1256(4)$ & $1509(5)$ & $6581(2)$ & $13(1)$ \\
$\mathrm{C}(17)$ & $-2568(4)$ & $2977(5)$ & $6631(3)$ & $16(1)$ \\
$\mathrm{C}(18)$ & $-4156(4)$ & $2169(5)$ & $6109(3)$ & $15(1)$ \\
$\mathrm{C}(19)$ & $-1376(4)$ & $3952(6)$ & $9343(3)$ & $23(1)$ \\
$\mathrm{C}(20)$ & $-4125(4)$ & $1884(6)$ & $5051(3)$ & $22(1)$ \\
& & & & \\
\hline
\end{tabular}
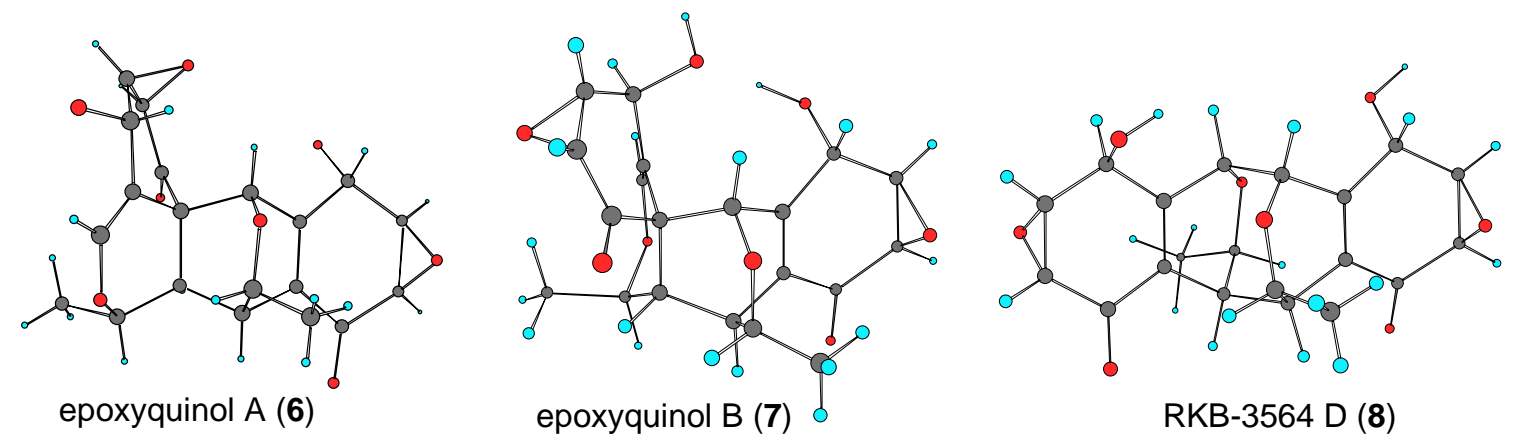

Figure. X-ray crystal structures of $6,{ }^{S 1} 7$, and 8

${ }^{\text {S1 }}$ Obtained from the Cambridge Structural Database (CSD Refcode: MOXHOW). 


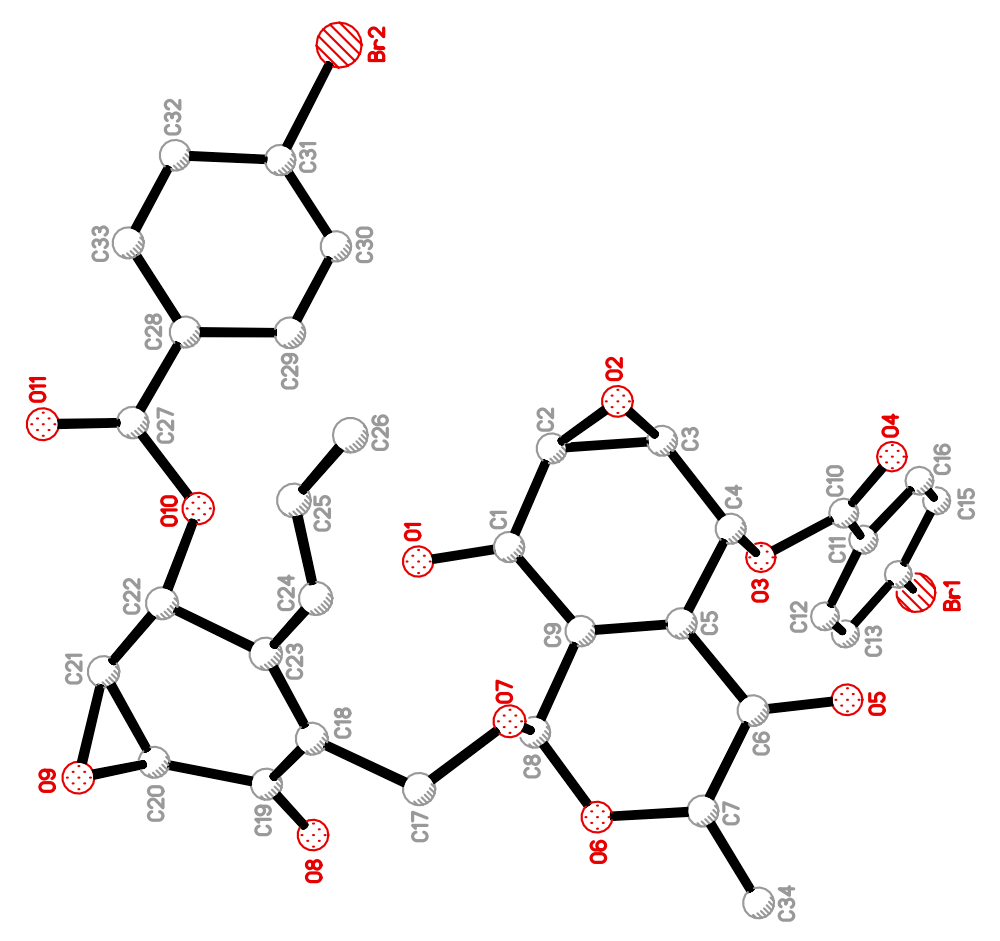

Crystals of compound 32 suitable for x-ray analysis were obtained by slow evaporation from Hexane/ $\mathrm{CH}_{2} \mathrm{Cl}_{2}$ /Ether. Crystallographic data have been deposited with the Cambridge Crystallographic Data Centre (CCDC \#257158). Copies of the data can be obtained free of charge on application to the CCDC, 12 Union Road, Cambridge CB21EZ, UK (fax: (+44)-1223-336-033; e-mail: deposit@ccdc.cam.ac.uk.

Table 1. Crystal data and structure refinement for 32.

Identification code

Empirical formula

Formula weight

Temperature

Wavelength

Crystal system

32

C34 H26 Br2 O11

770.37

173(2) K

$0.71073 \AA$

Space group

Orthorhombic

P2(1)2(1)2(1) 


$\begin{array}{lll}\text { Unit cell dimensions } & \begin{array}{l}\mathrm{a}=8.0070(16) \AA \\ \mathrm{b}=10.307(2) \AA\end{array} & \alpha=90^{\circ} . \\ & \mathrm{c}=41.274(8) \AA & \beta=90^{\circ} . \\ & 3406.4(12) \AA^{3} & \gamma=90^{\circ} . \\ \text { Volume } & 4 & \\ \text { Z } & 1.502 \mathrm{Mg} / \mathrm{m}^{3} & \\ \text { Density (calculated) } & 2.438 \mathrm{~mm}^{-1} & \\ \text { Absorption coefficient } & 1552 \\ \text { F(000) } & 0.40 \times 0.20 \times 0.02 \mathrm{~mm}^{3} \\ \text { Crystal size } & 2.59 \text { to } 20.88^{\circ} . \\ \text { Theta range for data collection } & -8<=\mathrm{h}<=6,-9<=\mathrm{k}<=10,-41<=1<=41 \\ \text { Index ranges } & 10236 \\ \text { Reflections collected } & 3547[\mathrm{R}(\mathrm{int})=0.0661] \\ \text { Independent reflections } & 99.1 \% \\ \text { Completeness to theta }=20.88^{\circ} & \mathrm{SADABS} \\ \text { Absorption correction } & 0.9529 \text { and } 0.5421 \\ \text { Max. and min. transmission } & \text { Full-matrix least-squares on } \mathrm{F}^{2} \\ \text { Refinement method } & 3547 / 0 / 440 \\ \text { Data / restraints / parameters } & 0.909 \\ \text { Goodness-of-fit on } \mathrm{F}^{2} & \mathrm{R} 1=0.0481, \mathrm{wR} 2=0.0986 \\ \text { Final R indices [I } 2 \text { sigma(I)] } & \mathrm{R} 1=0.0739, \mathrm{wR} 2=0.1095 \\ \mathrm{R} \text { indices (all data) } & 0.017(15) \\ \text { Absolute structure parameter } & 0.321 \text { and }-0.329 \text { e. } \AA^{-3} \\ \text { Largest diff. peak and hole } & \end{array}$

Table 2. Atomic coordinates ( $\left.\times 10^{4}\right)$ and equivalent isotropic displacement parameters $\left(\AA^{2} \times 10^{3}\right)$ for 32. $U(e q)$ is defined as one third of the trace of the orthogonalized $U^{i j}$ tensor.

\begin{tabular}{lrrrr}
\hline & $\mathrm{x}$ & $\mathrm{y}$ & $\mathrm{z}$ & $\mathrm{U}(\mathrm{eq})$ \\
\hline $\mathrm{Br}(1)$ & $1513(2)$ & $9124(1)$ & $10582(1)$ & $66(1)$ \\
$\mathrm{Br}(2)$ & $10776(1)$ & $6848(1)$ & $7922(1)$ & $43(1)$ \\
$\mathrm{O}(1)$ & $5537(9)$ & $2461(7)$ & $8715(2)$ & $46(2)$ \\
$\mathrm{O}(2)$ & $3795(8)$ & $5152(6)$ & $8383(1)$ & $44(2)$ \\
$\mathrm{O}(3)$ & $1736(7)$ & $5719(5)$ & $9175(1)$ & $27(2)$ \\
$\mathrm{O}(4)$ & $597(8)$ & $7552(5)$ & $8980(1)$ & $30(2)$ \\
$\mathrm{O}(5)$ & $-1359(8)$ & $4155(6)$ & $8960(1)$ & $36(2)$ \\
$\mathrm{O}(6)$ & $1021(8)$ & $1255(5)$ & $9068(1)$ & $30(2)$ \\
$\mathrm{O}(7)$ & $2138(6)$ & $970(5)$ & $8543(1)$ & $26(1)$ \\
$\mathrm{O}(8)$ & $4719(8)$ & $-913(6)$ & $9066(2)$ & $44(2)$ \\
$\mathrm{O}(9)$ & $7181(7)$ & $-2549(6)$ & $8512(2)$ & $43(2)$ \\
$\mathrm{O}(10)$ & $7872(7)$ & $795(5)$ & $8219(1)$ & $25(1)$ \\
$\mathrm{O}(11)$ & $10004(7)$ & $211(6)$ & $7894(1)$ & $31(2)$ \\
$\mathrm{C}(1)$ & $4455(13)$ & $3278(9)$ & $8725(2)$ & $28(2)$ \\
$\mathrm{C}(2)$ & $4804(11)$ & $4640(9)$ & $8648(2)$ & $30(3)$ \\
$\mathrm{C}(3)$ & $3464(13)$ & $5601(9)$ & $8703(2)$ & $34(3)$ \\
$\mathrm{C}(4)$ & $1820(11)$ & $5239(8)$ & $8843(2)$ & $27(2)$ \\
$\mathrm{C}(5)$ & $1524(12)$ & $3782(8)$ & $8866(2)$ & $21(2)$ \\
$\mathrm{C}(6)$ & $-214(12)$ & $3370(10)$ & $8949(2)$ & $26(2)$ \\
$\mathrm{C}(7)$ & $-481(11)$ & $1935(9)$ & $8994(2)$ & $26(2)$ \\
$\mathrm{C}(8)$ & $2355(12)$ & $1493(8)$ & $8860(2)$ & $27(2)$ \\
$\mathrm{C}(9)$ & $2718(11)$ & $2912(8)$ & $8813(2)$ & $19(2)$ \\
$\mathrm{C}(10)$ & $1095(11)$ & $6945(9)$ & $9207(2)$ & $27(2)$ \\
$\mathrm{C}(11)$ & $1125(12)$ & $7401(9)$ & $9540(2)$ & $29(3)$ \\
$\mathrm{C}(12)$ & $1297(14)$ & $6588(10)$ & $9799(2)$ & $55(3)$ \\
$\mathrm{C}(13)$ & $1351(16)$ & $7102(10)$ & $10121(2)$ & $64(4)$ \\
$\mathrm{C}(14)$ & $1290(13)$ & $8414(10)$ & $10159(2)$ & $42(3)$ \\
& & & &
\end{tabular}




$\begin{array}{lrrrr}\mathrm{C}(15) & 1112(13) & 9235(9) & 9905(2) & 52(3) \\ \mathrm{C}(16) & 1052(13) & 8731(9) & 9594(2) & 45(3) \\ \mathrm{C}(17) & 2581(11) & -389(8) & 8526(2) & 28(2) \\ \mathrm{C}(18) & 4458(12) & -597(8) & 8495(2) & 29(2) \\ \mathrm{C}(19) & 5308(12) & -1000(9) & 8802(3) & 30(3) \\ \mathrm{C}(20) & 7006(12) & -1576(9) & 8767(2) & 34(3) \\ \mathrm{C}(21) & 7943(12) & -1303(8) & 8468(2) & 28(2) \\ \mathrm{C}(22) & 7202(11) & -522(8) & 8201(2) & 27(2) \\ \mathrm{C}(23) & 5304(11) & -365(8) & 8217(2) & 23(2) \\ \mathrm{C}(24) & 4503(11) & 120(8) & 7924(2) & 35(2) \\ \mathrm{C}(25) & 5159(14) & 629(12) & 7669(2) & 63(4) \\ \mathrm{C}(26) & 4338(14) & 1166(13) & 7371(2) & 80(4) \\ \mathrm{C}(27) & 9252(12) & 1045(9) & 8036(2) & 23(2) \\ \mathrm{C}(28) & 9621(12) & 2436(9) & 8029(2) & 20(2) \\ \mathrm{C}(29) & 8547(13) & 3360(9) & 8157(2) & 33(3) \\ \mathrm{C}(30) & 8894(14) & 4685(9) & 8131(2) & 43(3) \\ \mathrm{C}(31) & 10275(12) & 5064(9) & 7969(2) & 32(3) \\ \mathrm{C}(32) & 11422(12) & 4167(10) & 7847(2) & 38(3) \\ \mathrm{C}(33) & 11038(11) & 2836(8) & 7874(2) & 30(2) \\ \mathrm{C}(34) & -1697(11) & 1672(9) & 9266(2) & 42(3) \\ \mathrm{C}(1 \mathrm{~S}) & -190(40) & 13460(30) & 10111(7) & 239(11) \\ \mathrm{C}(2 S) & 1300(30) & 12420(20) & 9987(5) & 169(7) \\ \mathrm{C}(3 \mathrm{~S}) & 2650(40) & 13200(30) & 9777(7) & 246(12) \\ \mathrm{C}(4 \mathrm{~S}) & 3870(30) & 12440(20) & 9692(5) & 147(7) \\ & & & & \end{array}$




\section{$\underline{\text { X-ray Crystal Structure of } 55}$}

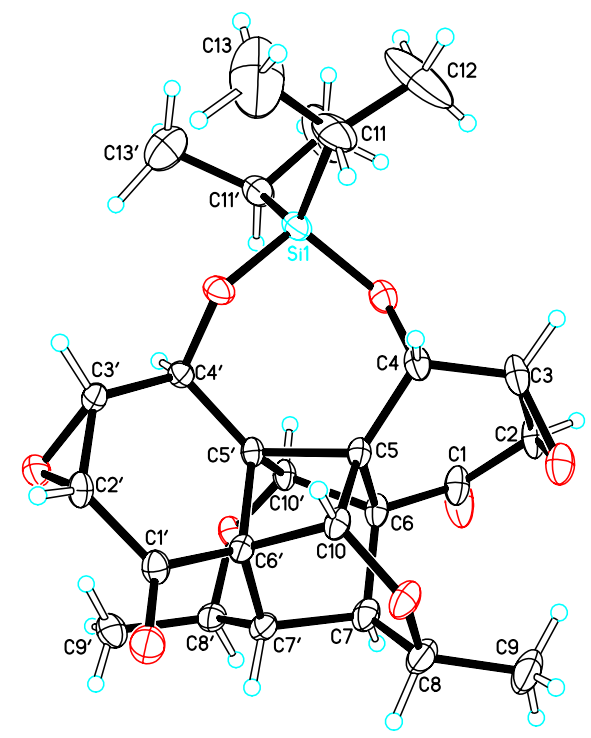

Crystals of compound 55 suitable for x-ray analysis were obtained by slow evaporation from $\mathrm{CH}_{2} \mathrm{Cl}_{2} / \mathrm{CH}_{3} \mathrm{CN}$. Crystallographic data have been deposited with the Cambridge Crystallographic Data Centre (CCDC \#220118). Copies of the data can be obtained free of charge on application to the CCDC, 12 Union Road, Cambridge CB21EZ, UK (fax: (+44)-1223-336-033; e-mail: deposit@ccdc.cam.ac.uk.

Table 1. Crystal data and structure refinement for compound 55.

Identification code

Empirical formula

Formula weight

Temperature

Wavelength

Crystal system

Space group

Unit cell dimensions

Volume

Z

Density (calculated)

Absorption coefficient

$\mathrm{F}(000)$

Crystal size

Theta range for data collection

Index ranges

Reflections collected

Independent reflections

Completeness to theta $=29.58^{\circ}$

Absorption correction

Max. and min. transmission

Refinement method
55

$\mathrm{C} 26 \mathrm{H} 32 \mathrm{O} 8 \mathrm{Si} * \mathrm{CHCl} 3$

619.97

173(2) K

$0.71073 \AA$

Monoclinic

P2(1)

$\begin{array}{ll}\mathrm{a}=14.3118(12) \AA & \alpha=90^{\circ} . \\ \mathrm{b}=7.8572(7) \AA & \beta=114.959(2)^{\circ} . \\ \mathrm{c}=14.4216(12) \AA & \gamma=90^{\circ} .\end{array}$

1470.3(2) $\AA^{3}$

2

$1.400 \mathrm{Mg} / \mathrm{m}^{3}$

$0.399 \mathrm{~mm}^{-1}$

648

$0.50 \times 0.30 \times 0.25 \mathrm{~mm}^{3}$

2.64 to $29.58^{\circ}$.

$-19<=\mathrm{h}<=19,-10<=\mathrm{k}<=10,-18<=1<=19$

14222

$7273[\mathrm{R}(\mathrm{int})=0.0274]$

$92.0 \%$

SADABS

0.9068 and 0.8253

Full-matrix least-squares on $\mathrm{F}^{2}$ 
Data / restraints / parameters

Goodness-of-fit on $\mathrm{F}^{2}$

Final $\mathrm{R}$ indices $[\mathrm{I}>2$ sigma(I)]

$\mathrm{R}$ indices (all data)

Absolute structure parameter

Largest diff. peak and hole
$7273 / 1 / 449$

1.023

$\mathrm{R} 1=0.0480, \mathrm{wR} 2=0.1208$

$\mathrm{R} 1=0.0597, \mathrm{wR} 2=0.1283$

$0.01(8)$

0.425 and -0.421 e. $\AA^{-3}$

Table 2. Atomic coordinates $\left(\mathrm{x} 10^{4}\right)$ and equivalent isotropic displacement parameters $\left(\AA^{2} \times 10^{3}\right)$ for 55. $U(e q)$ is defined as one third of the trace of the orthogonalized $U^{i j}$ tensor.

\begin{tabular}{|c|c|c|c|c|}
\hline & $\mathrm{x}$ & $\mathrm{y}$ & $\mathrm{Z}$ & $\mathrm{U}(\mathrm{eq})$ \\
\hline $\mathrm{Si}(1)$ & $8080(1)$ & 9334(1) & $2227(1)$ & $25(1)$ \\
\hline $\mathrm{O}\left(1^{\prime}\right)$ & $6010(2)$ & $6363(3)$ & $-2476(2)$ & $37(1)$ \\
\hline $\mathrm{O}(1)$ & $7797(2)$ & $14618(2)$ & $-428(2)$ & $50(1)$ \\
\hline $\mathrm{O}\left(2^{\prime}\right)$ & 4934(1) & $7035(2)$ & $-831(1)$ & $26(1)$ \\
\hline $\mathrm{O}(2)$ & $9782(1)$ & $11615(2)$ & $7(2)$ & $32(1)$ \\
\hline $\mathrm{O}(3)$ & $8522(1)$ & $10680(2)$ & $1623(1)$ & $26(1)$ \\
\hline $\mathrm{O}\left(3^{\prime}\right)$ & $7346(1)$ & $7989(2)$ & $1349(1)$ & $25(1)$ \\
\hline $\mathrm{O}\left(4^{\prime}\right)$ & $5562(1)$ & $11896(2)$ & $-1172(1)$ & $24(1)$ \\
\hline $\mathrm{O}(4)$ & $8259(1)$ & $8963(2)$ & $-1674(2)$ & $30(1)$ \\
\hline$C\left(1^{\prime}\right)$ & $6100(2)$ & $7023(3)$ & $-1685(2)$ & $23(1)$ \\
\hline $\mathrm{C}(1)$ & $8162(2)$ & $13196(3)$ & $-302(2)$ & $30(1)$ \\
\hline $\mathrm{C}\left(2^{\prime}\right)$ & $5741(2)$ & $6128(3)$ & $-991(2)$ & $24(1)$ \\
\hline $\mathrm{C}(2)$ & $9286(2)$ & $12954(3)$ & $335(2)$ & $32(1)$ \\
\hline$C\left(3^{\prime}\right)$ & $5918(2)$ & $6845(3)$ & $12(2)$ & $22(1)$ \\
\hline $\mathrm{C}(3)$ & $9647(2)$ & $11354(3)$ & $918(2)$ & $31(1)$ \\
\hline$C\left(4^{\prime}\right)$ & $6487(2)$ & $8489(3)$ & $433(2)$ & $19(1)$ \\
\hline $\mathrm{C}(4)$ & $8855(2)$ & $10057(3)$ & $878(2)$ & $25(1)$ \\
\hline$C\left(5^{\prime}\right)$ & $6798(2)$ & $9514(3)$ & $-295(2)$ & $18(1)$ \\
\hline$C(5)$ & $7938(2)$ & $9922(3)$ & $-167(2)$ & $20(1)$ \\
\hline$C\left(6^{\prime}\right)$ & $6613(2)$ & $8729(3)$ & $-1367(2)$ & $20(1)$ \\
\hline $\mathrm{C}(6)$ & $7504(2)$ & $11647(3)$ & $-729(2)$ & $22(1)$ \\
\hline$C(7)$ & $7111(2)$ & $11379(3)$ & $-1904(2)$ & $27(1)$ \\
\hline$C\left(7^{\prime}\right)$ & $6196(2)$ & 10151(3) & $-2182(2)$ & $24(1)$ \\
\hline $\mathrm{C}\left(8^{\prime}\right)$ & $5278(2)$ & $11127(3)$ & $-2177(2)$ & $26(1)$ \\
\hline $\mathrm{C}(8)$ & $7868(2)$ & $10515(4)$ & $-2254(2)$ & $33(1)$ \\
\hline$C\left(9^{\prime}\right)$ & $4294(2)$ & $10115(4)$ & $-2477(2)$ & $31(1)$ \\
\hline $\mathrm{C}(9)$ & $8750(2)$ & $11573(5)$ & $-2267(3)$ & $46(1)$ \\
\hline$C(10)$ & $7830(2)$ & $8593(3)$ & $-978(2)$ & $22(1)$ \\
\hline $\mathrm{C}\left(10^{\prime}\right)$ & $6551(2)$ & $11433(3)$ & $-455(2)$ & $20(1)$ \\
\hline $\mathrm{C}\left(11^{\prime}\right)$ & $7354(2)$ & $10716(4)$ & $2747(2)$ & $32(1)$ \\
\hline $\mathrm{C}(11)$ & $9151(2)$ & $7967(4)$ & $3115(3)$ & $45(1)$ \\
\hline$C\left(12^{\prime}\right)$ & $8054(3)$ & $11890(6)$ & $3599(3)$ & $70(1)$ \\
\hline$C(12)$ & $10043(3)$ & $8914(7)$ & $3861(4)$ & $97(2)$ \\
\hline$C\left(13^{\prime}\right)$ & $6629(3)$ & $9717(6)$ & $3081(4)$ & $56(1)$ \\
\hline$C(13)$ & $8852(4)$ & $6437(10)$ & $3473(7)$ & $160(4)$ \\
\hline $\mathrm{Cl}(1)$ & $4124(1)$ & $2777(3)$ & 4889(1) & $109(1)$ \\
\hline $\mathrm{Cl}(2)$ & 1939(1) & 2311(3) & $4072(1)$ & $123(1)$ \\
\hline $\mathrm{Cl}(3)$ & $3286(2)$ & $-628(3)$ & $4432(1)$ & $122(1)$ \\
\hline$C(1 S)$ & $3086(4)$ & $1532(8)$ & $4068(3)$ & $85(2)$ \\
\hline
\end{tabular}




\section{$\underline{\text { X-ray Crystal Structure of } 63}$}

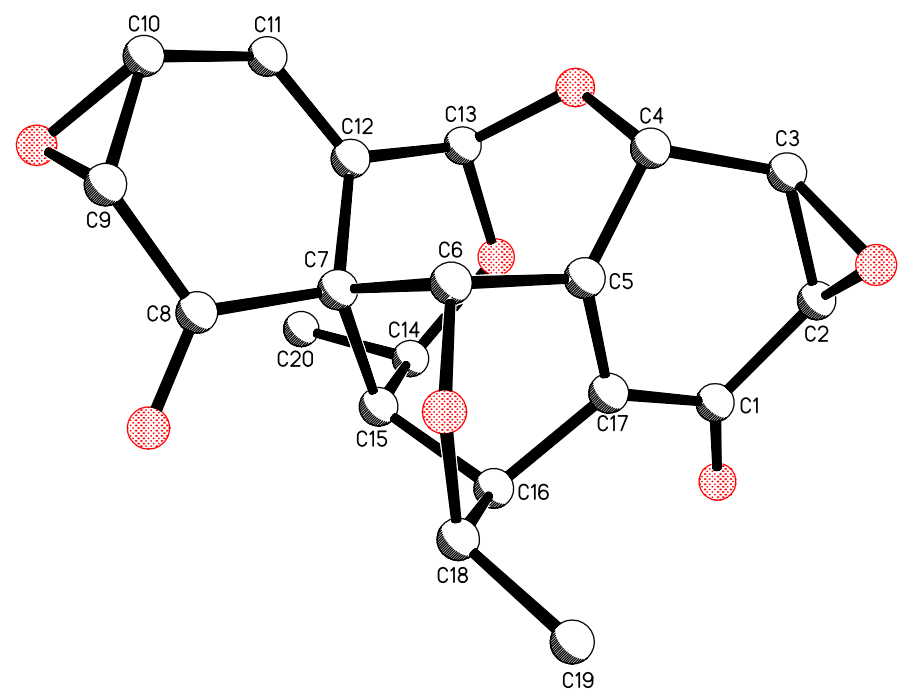

Crystals of compound 63 suitable for x-ray analysis were obtained by slow evaporation from $\mathrm{CH}_{2} \mathrm{Cl}_{2} / \mathrm{PhCH}_{3}$. Crystallographic data have been deposited with the Cambridge Crystallographic Data Centre (CCDC \#257159). Copies of the data can be obtained free of charge on application to the CCDC, 12 Union Road, Cambridge CB21EZ, UK (fax: (+44)-1223-336-033; e-mail: deposit@ccdc.cam.ac.uk.

Table 1. Crystal data and structure refinement for 63.

Identification code

Empirical formula

Formula weight

Temperature

Wavelength

Crystal system

Space group

Unit cell dimensions

Volume

Z

Density (calculated)

Absorption coefficient

$\mathrm{F}(000)$

Crystal size

Theta range for data collection

Index ranges

Reflections collected

Independent reflections

Completeness to theta $=28.28^{\circ}$

Absorption correction

\section{3}

C20 H18 O7 + (C7 H8)

462.48

173(2) K

$0.71073 \AA$

Orthorhombic

P2(1)2(1)2(1)

$\mathrm{a}=10.216(2) \AA$

$\mathrm{b}=11.299(2) \AA$

$\mathrm{c}=19.715(4) \AA$

2275.7(8) $\AA^{3}$

4

$1.350 \mathrm{Mg} / \mathrm{m}^{3}$

$0.097 \mathrm{~mm}^{-1}$

976

$0.40 \times 0.15 \times 0.10 \mathrm{~mm}^{3}$

2.25 to $28.28^{\circ}$.

$-13<=\mathrm{h}<=12,-15<=\mathrm{k}<=10,-26<=1<=26$

21536

$5641[\mathrm{R}(\mathrm{int})=0.0341]$

$99.9 \%$

SADABS $\alpha=90^{\circ}$.

$\beta=90^{\circ}$.

$\gamma=90^{\circ}$. 
Max. and min. transmission

Refinement method

Data / restraints / parameters

Goodness-of-fit on $\mathrm{F}^{2}$

Final $\mathrm{R}$ indices $[\mathrm{I}>2$ sigma(I)]

$\mathrm{R}$ indices (all data)

Absolute structure parameter

Largest diff. peak and hole
0.9903 and 0.9621

Full-matrix least-squares on $\mathrm{F}^{2}$

$5641 / 0 / 379$

1.024

$\mathrm{R} 1=0.0422, \mathrm{wR} 2=0.1033$

$\mathrm{R} 1=0.0540, \mathrm{wR} 2=0.1095$

$-0.6(8)$

0.253 and -0.226 e. $\AA^{-3}$

Table 2. Atomic coordinates $\left(\mathrm{x} 10^{4}\right)$ and equivalent isotropic displacement parameters $\left(\AA^{2} \times 10^{3}\right)$ for 63. $U(\mathrm{eq})$ is defined as one third of the trace of the orthogonalized $\mathrm{U}^{\mathrm{ij}}$ tensor.

\begin{tabular}{|c|c|c|c|c|}
\hline & $\mathrm{x}$ & $\mathrm{y}$ & $\mathrm{z}$ & $\mathrm{U}(\mathrm{eq})$ \\
\hline $\mathrm{O}(1)$ & $6005(1)$ & $6224(1)$ & $1321(1)$ & $42(1)$ \\
\hline $\mathrm{O}(2)$ & $7330(1)$ & $3845(1)$ & $409(1)$ & $39(1)$ \\
\hline $\mathrm{O}(3)$ & $5438(1)$ & $2277(1)$ & $1718(1)$ & $32(1)$ \\
\hline $\mathrm{O}(4)$ & $83(2)$ & $1607(1)$ & $1852(1)$ & $48(1)$ \\
\hline $\mathrm{O}(5)$ & $323(1)$ & $3758(2)$ & $847(1)$ & $66(1)$ \\
\hline $\mathrm{O}(6)$ & $4513(1)$ & 3964(1) & $2195(1)$ & $31(1)$ \\
\hline $\mathrm{O}(7)$ & $2790(1)$ & $3770(1)$ & $49(1)$ & $32(1)$ \\
\hline $\mathrm{C}(1)$ & $5962(2)$ & $5225(2)$ & 1091(1) & $29(1)$ \\
\hline $\mathrm{C}(2)$ & $7172(2)$ & $4483(2)$ & $1040(1)$ & $35(1)$ \\
\hline $\mathrm{C}(3)$ & $7024(2)$ & $3187(2)$ & $1007(1)$ & $33(1)$ \\
\hline$C(4)$ & $5679(2)$ & $2632(2)$ & $1017(1)$ & $27(1)$ \\
\hline$C(5)$ & $4665(2)$ & $3477(1)$ & $770(1)$ & $23(1)$ \\
\hline$C(6)$ & $3302(2)$ & $3074(2)$ & $590(1)$ & $26(1)$ \\
\hline$C(7)$ & $2469(2)$ & $3276(2)$ & $1257(1)$ & $27(1)$ \\
\hline $\mathrm{C}(8)$ & $1043(2)$ & $3017(2)$ & $1068(1)$ & $37(1)$ \\
\hline $\mathrm{C}(9)$ & $537(2)$ & $1782(2)$ & $1167(1)$ & $40(1)$ \\
\hline$C(10)$ & $1249(2)$ & $981(2)$ & $1613(1)$ & $41(1)$ \\
\hline $\mathrm{C}(11)$ & 2493(2) & $1374(2)$ & $1918(1)$ & $35(1)$ \\
\hline$C(12)$ & $3031(2)$ & $2410(2)$ & $1769(1)$ & $28(1)$ \\
\hline$C(13)$ & $4342(2)$ & $2733(2)$ & $2086(1)$ & $30(1)$ \\
\hline $\mathrm{C}(14)$ & $3357(2)$ & $4714(2)$ & $2148(1)$ & $32(1)$ \\
\hline$C(15)$ & $2670(2)$ & $4599(2)$ & $1460(1)$ & $26(1)$ \\
\hline$C(16)$ & $3437(2)$ & $5245(2)$ & $881(1)$ & $24(1)$ \\
\hline$C(17)$ & $4746(2)$ & $4651(2)$ & $860(1)$ & $23(1)$ \\
\hline $\mathrm{C}(18)$ & $2729(2)$ & $5027(2)$ & 207(1) & $31(1)$ \\
\hline C(19) & $3321(3)$ & $5691(2)$ & $-386(1)$ & $43(1)$ \\
\hline$C(20)$ & $2438(3)$ & $4496(3)$ & $2745(1)$ & $53(1)$ \\
\hline$C(1 S)$ & $3061(3)$ & $-1663(2)$ & 1681(1) & $54(1)$ \\
\hline $\mathrm{C}(2 \mathrm{~S})$ & $1928(5)$ & $-2235(3)$ & $1887(2)$ & $89(1)$ \\
\hline $\mathrm{C}(3 \mathrm{~S})$ & $1008(4)$ & $-2515(4)$ & $1410(5)$ & $135(3)$ \\
\hline $\mathrm{C}(4 \mathrm{~S})$ & $1164(6)$ & $-2244(5)$ & $788(5)$ & $147(3)$ \\
\hline $\mathrm{C}(5 \mathrm{~S})$ & $2214(5)$ & $-1675(3)$ & $572(1)$ & $88(1)$ \\
\hline $\mathrm{C}(6 \mathrm{~S})$ & $3229(3)$ & $-1361(2)$ & $1004(1)$ & $52(1)$ \\
\hline $\mathrm{C}(7 \mathrm{~S})$ & $4429(5)$ & $-743(3)$ & $769(3)$ & $150(3)$ \\
\hline
\end{tabular}




\section{NMR Spectra of Select Compounds (top: ${ }^{1} \mathrm{H}$ NMR, bottom: ${ }^{13} \mathrm{C}$ NMR)}
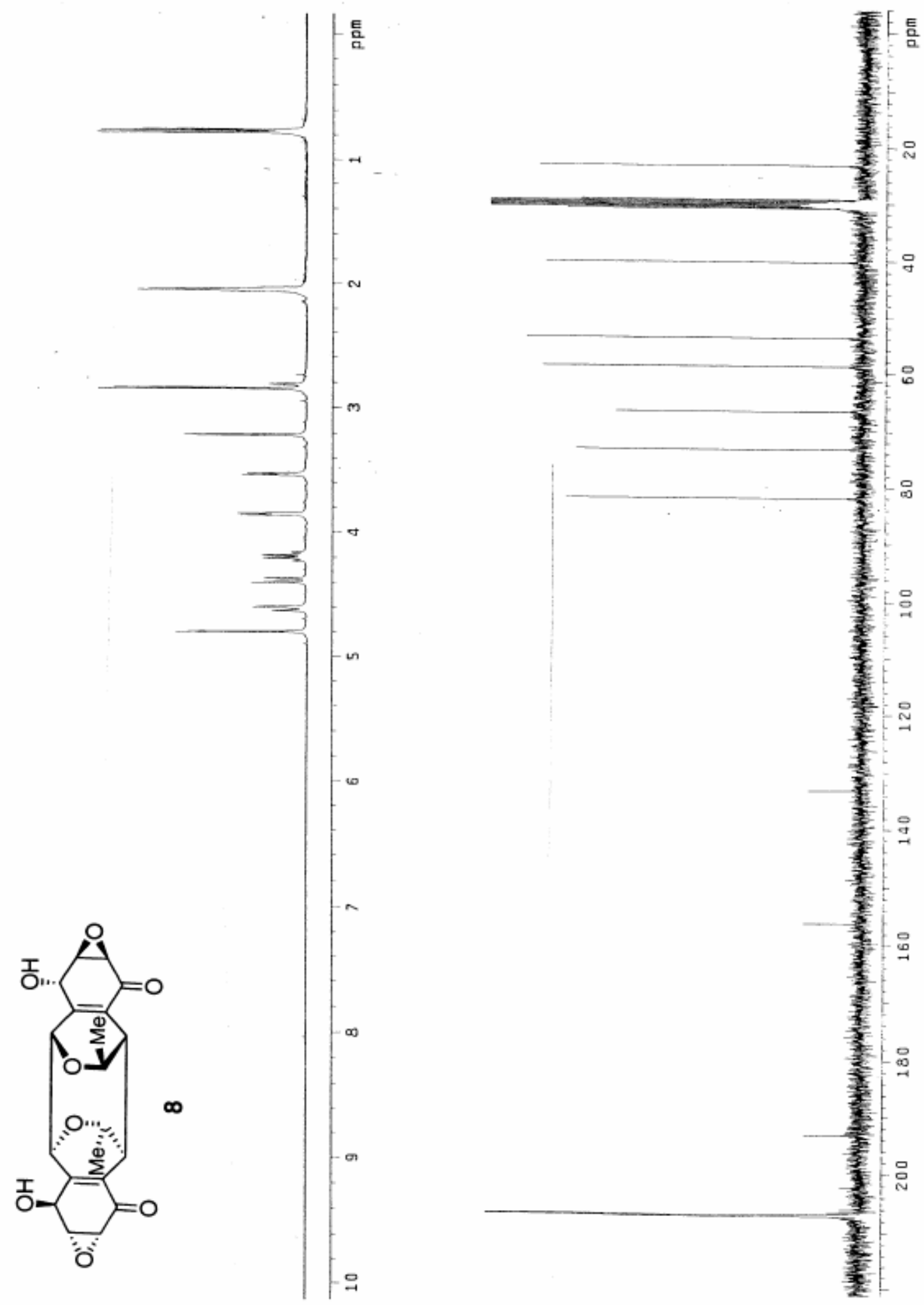
- S34 - 

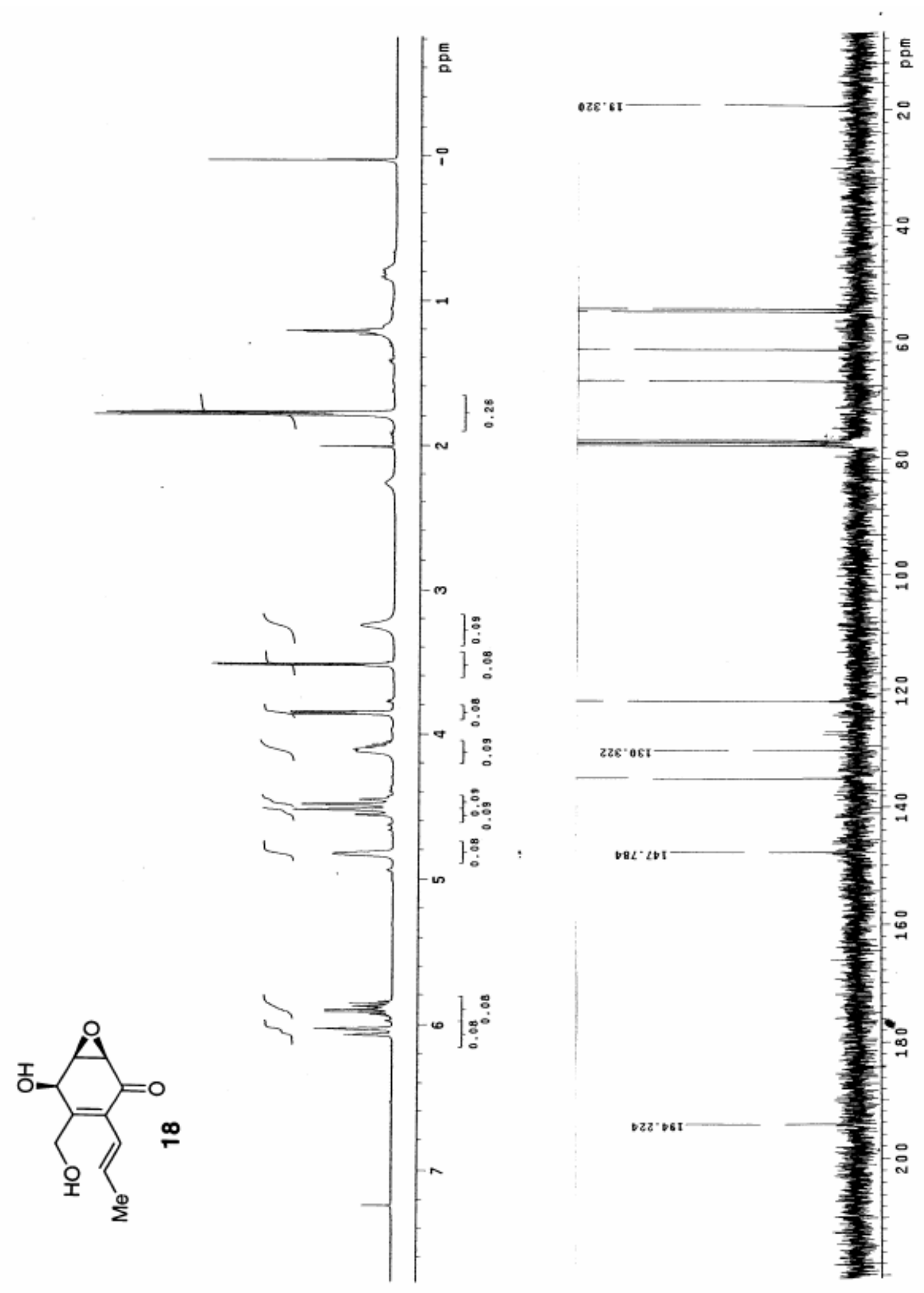

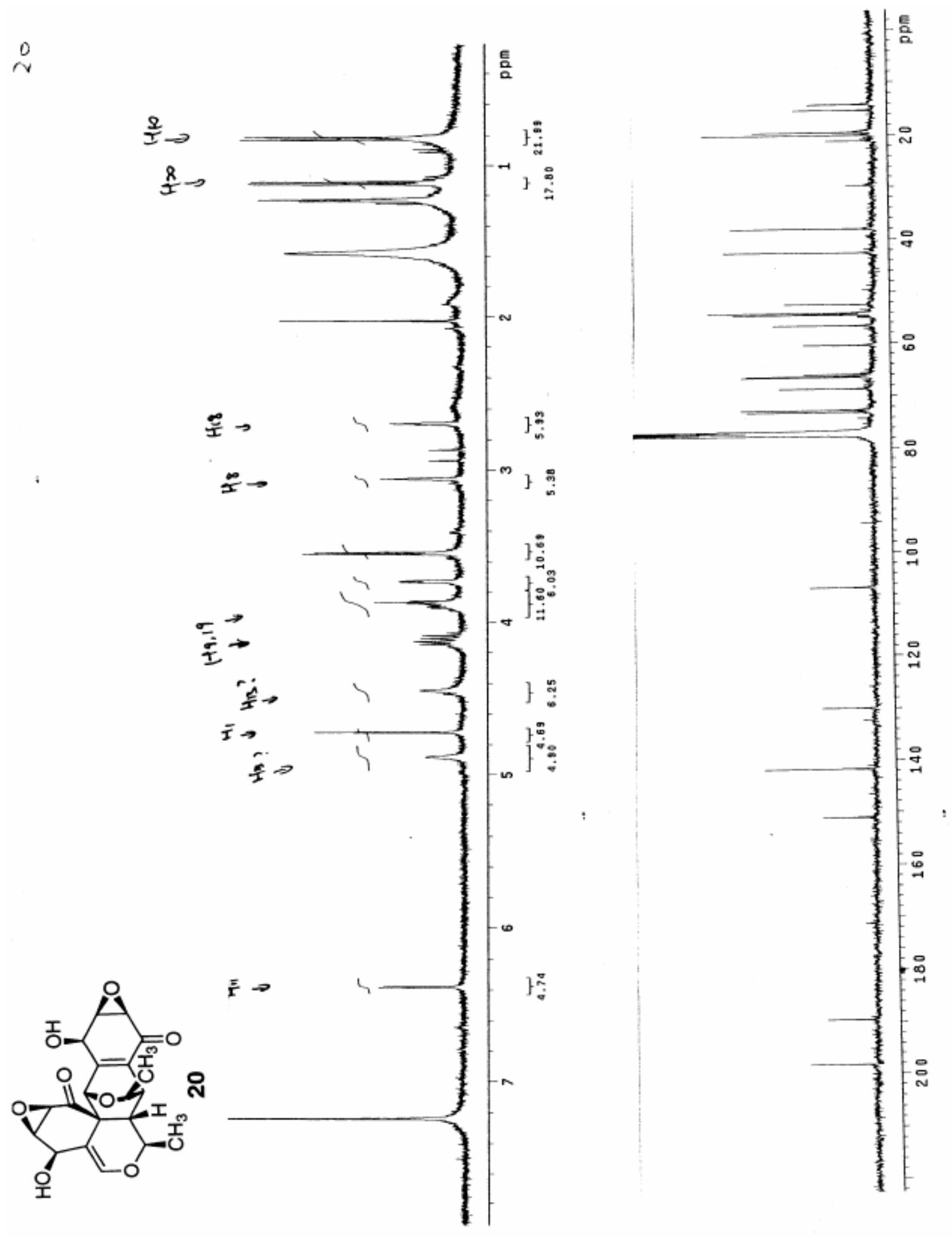


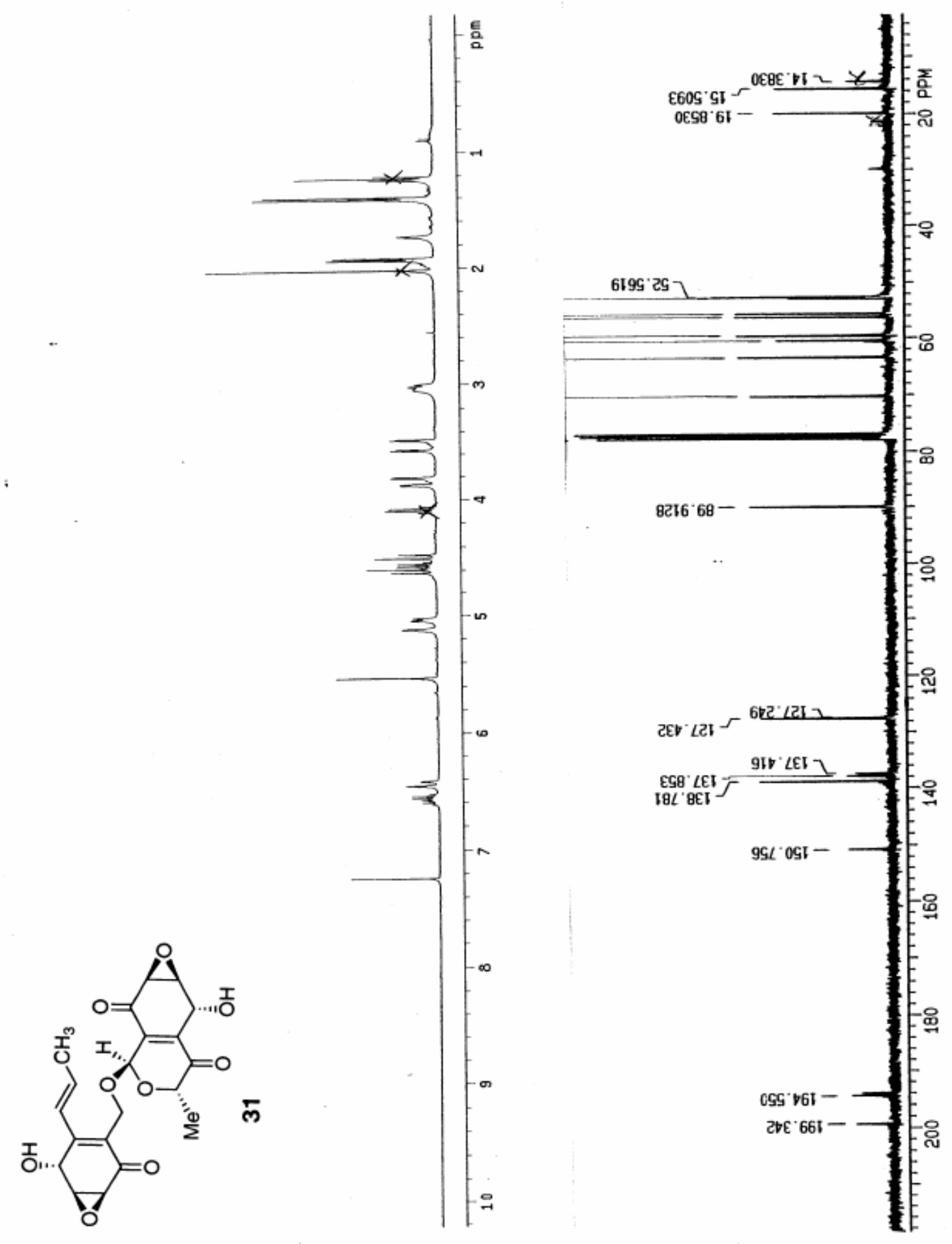



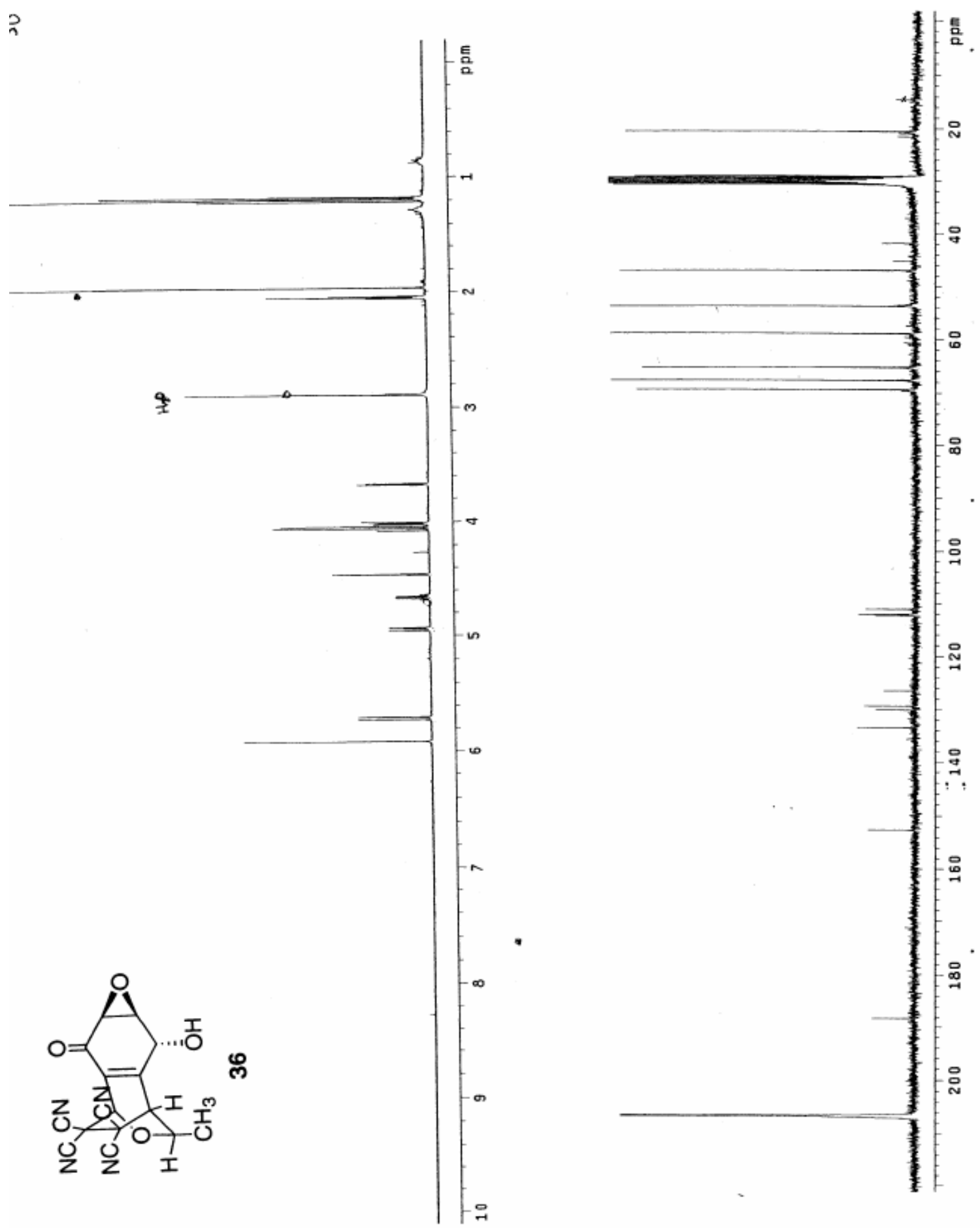

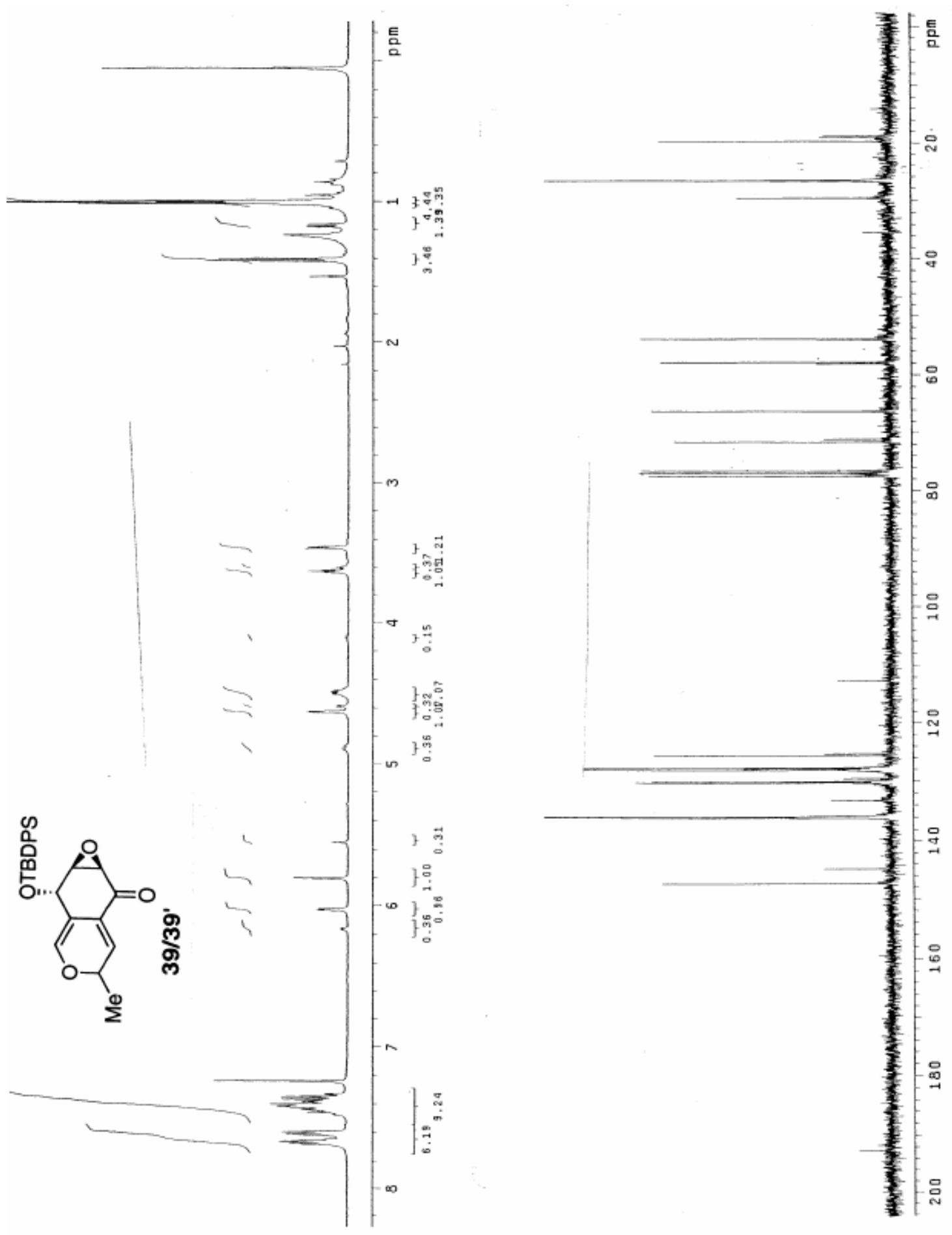


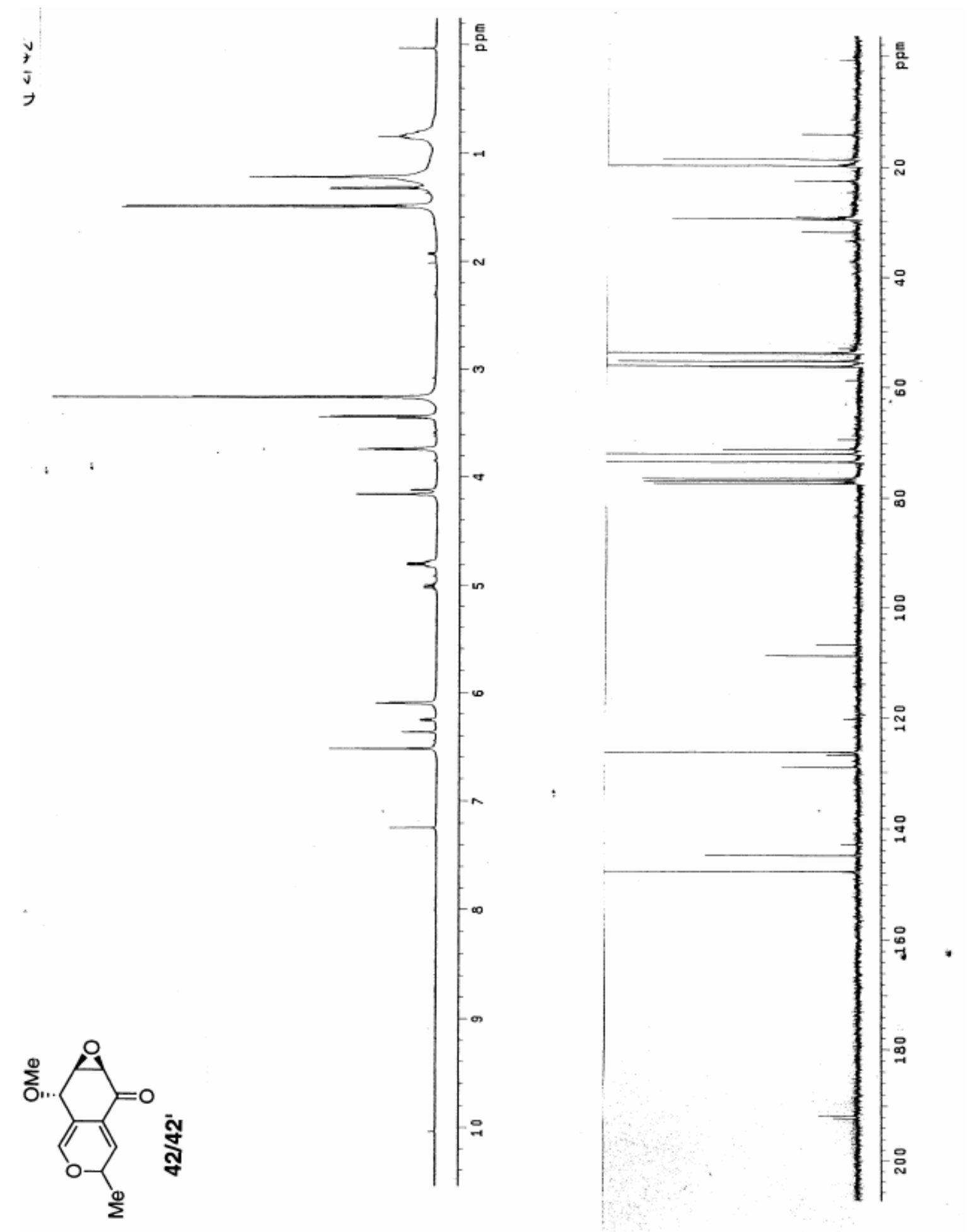



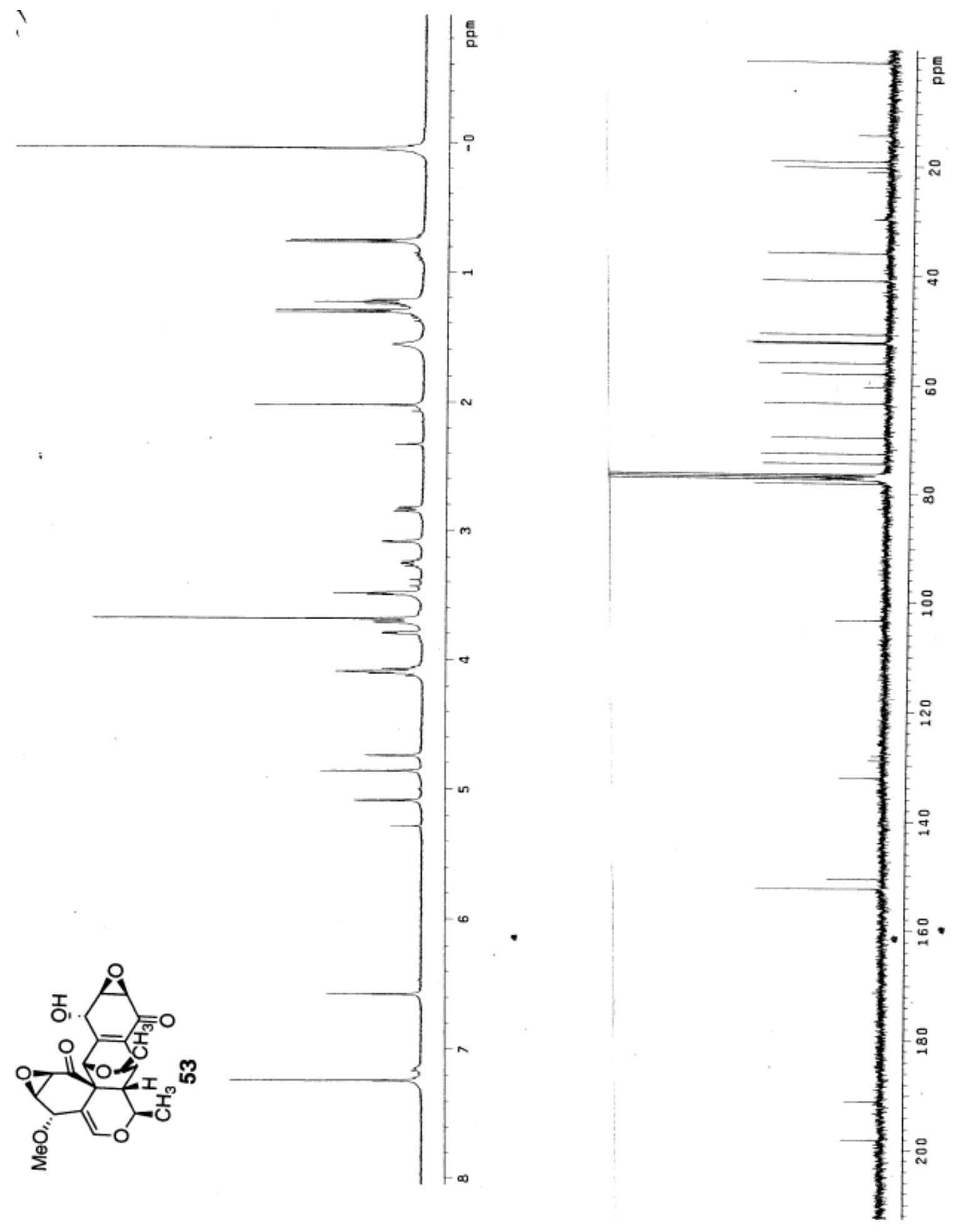


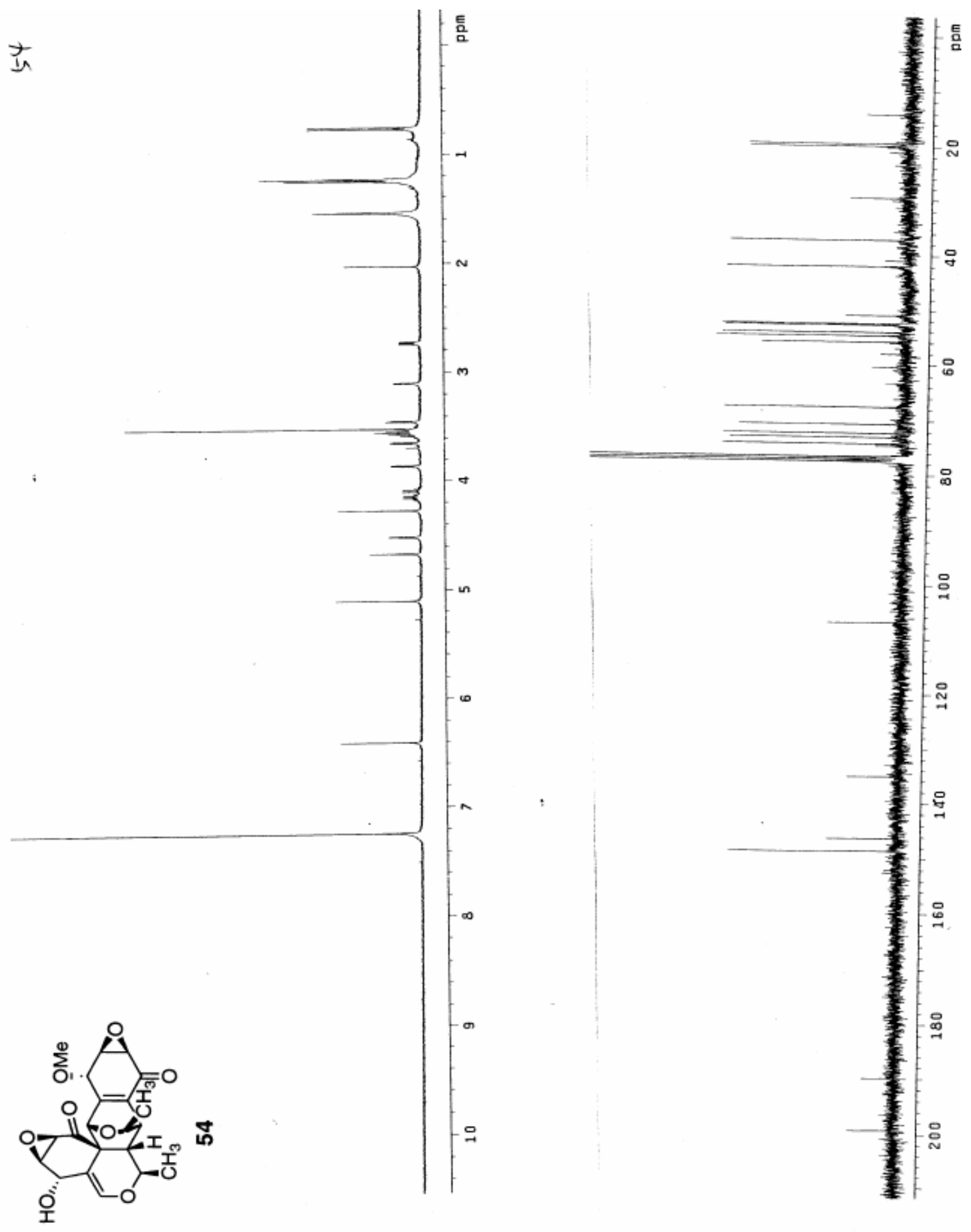




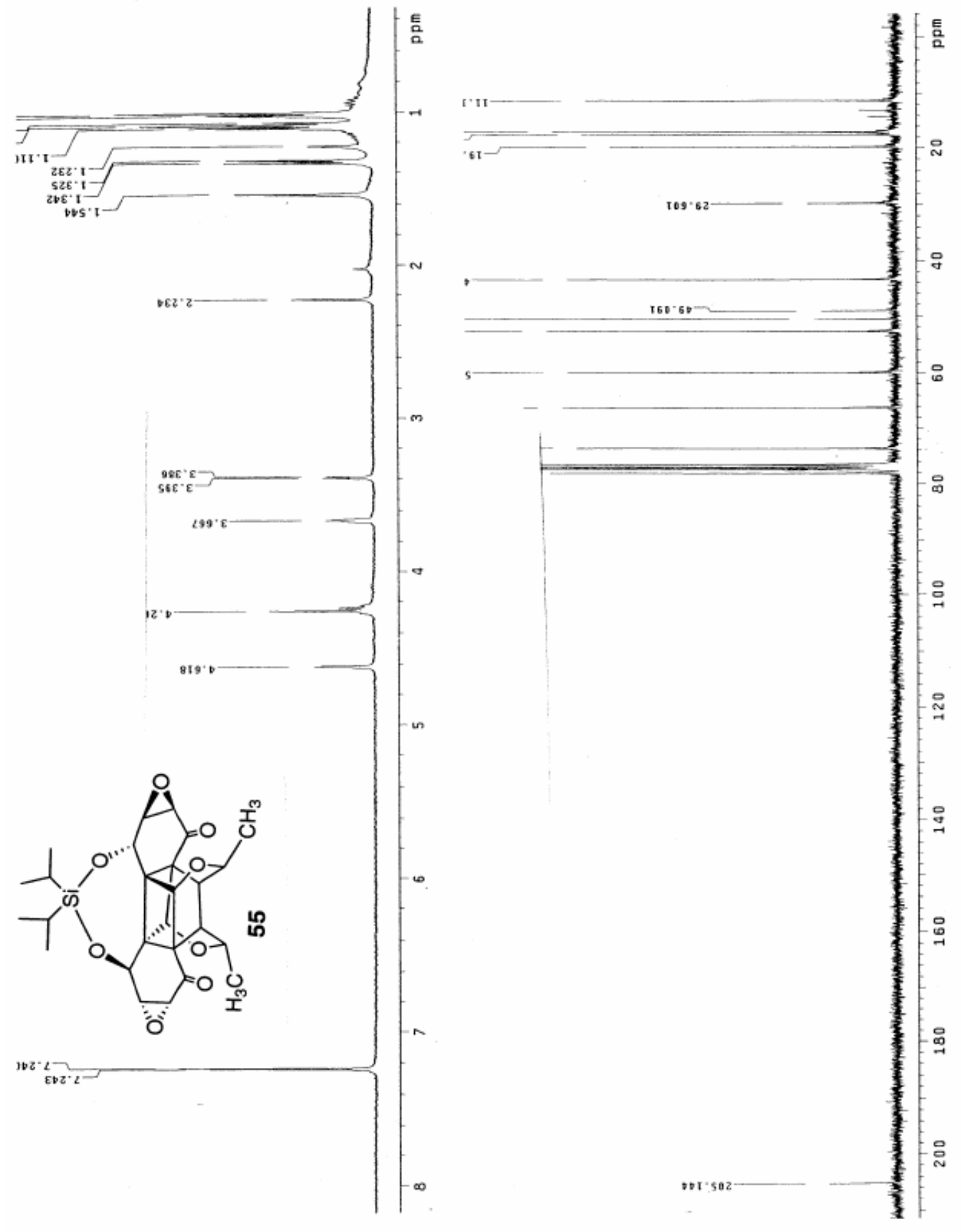



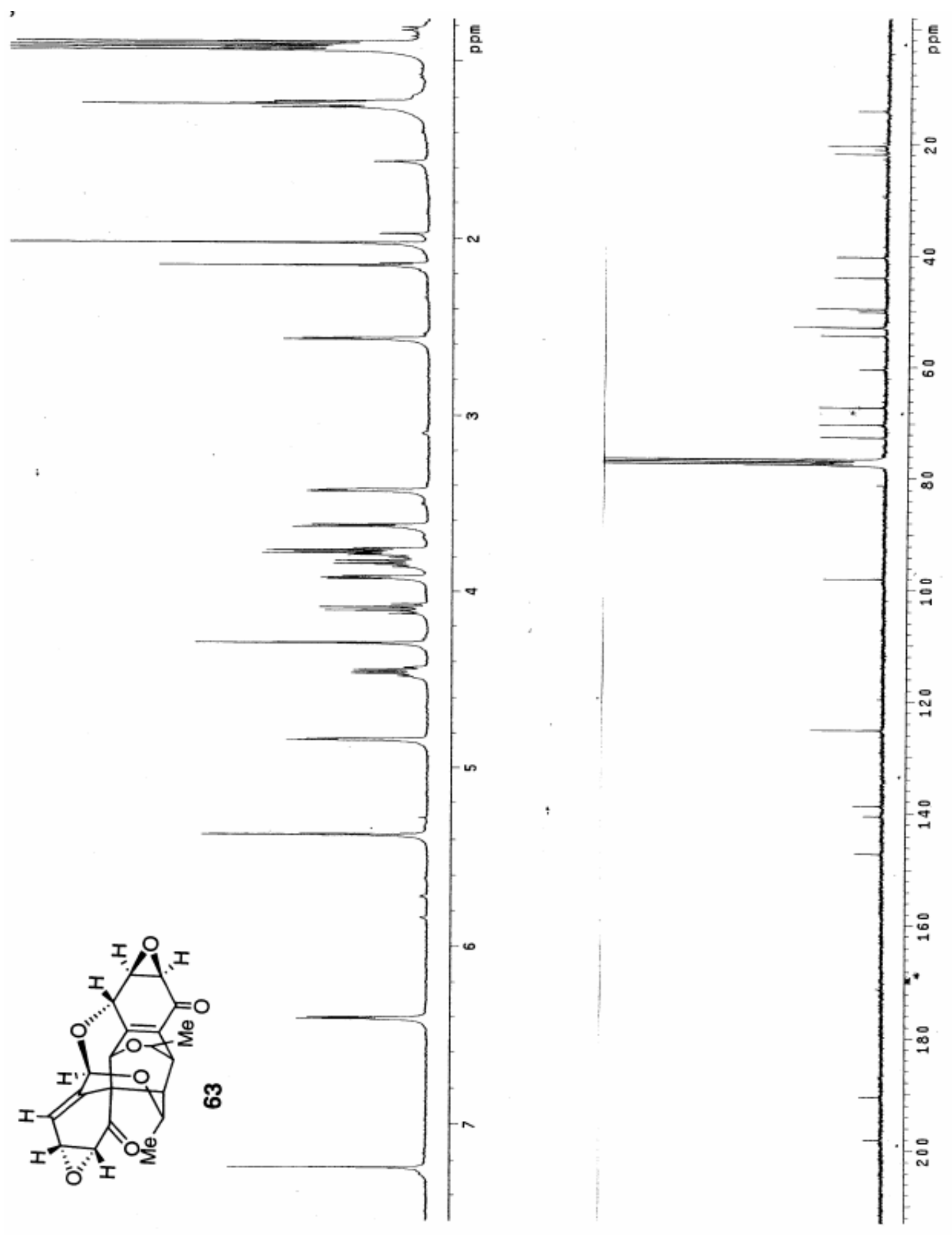

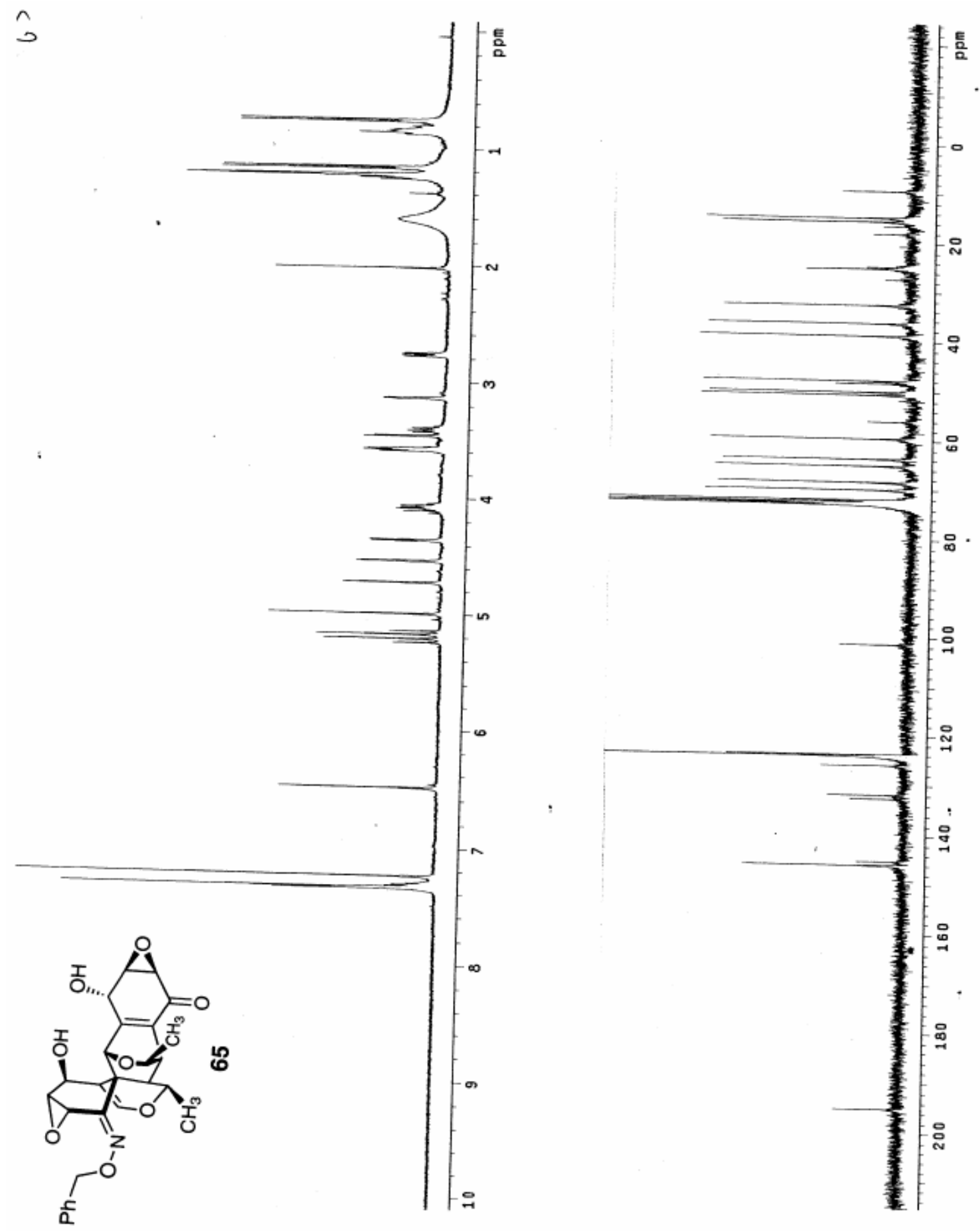